\title{
Energy transfer and chemical dynamics at solid surfaces: The special role of charge transfer
}

\author{
Alec M. Wodtke ${ }^{\mathrm{a}, *}$, Daniel Matsiev ${ }^{\mathrm{a}}$, Daniel J. Auerbach ${ }^{\mathrm{b}}$ \\ a Department of Chemistry and Biochemistry, University of California, Santa Barbara, CA 93106, USA \\ ${ }^{\mathrm{b}}$ Gas Reaction Technologies, 861 Ward Drive, Santa Barbara, CA 93111, USA
}

Commissioning Editor: H. Winter

This article is dedicated to the memory of my mother, Dana Wodtke.

\begin{abstract}
Molecular energy transfer processes at solid surfaces are profoundly important, influencing trapping, desorption, diffusion, and reactivity; in short, all of the elementary steps needed for surface chemistry to take place. In this paper we review recent progress in our understanding of energy transfer at surfaces with a particular emphasis on those phenomena, which are peculiar to solids with delocalized electronic structure, e.g. electronically nonadiabatic energy transfer. This area of study represents an area requiring significant extensions of our theoretical understanding, which is largely based on density functional theory. This review provides an overview of some of the experimental and theoretical tools presently being used in this field and a description of several illustrative examples of work that have helped to shape our understanding.

(c) 2008 Elsevier Ltd. All rights reserved.
\end{abstract}

Keywords: Energy transfer; Surface science; Chemical dynamics; Electronic nonadiabaticity; Born-Oppenheimer breakdown

\section{Contents}

1. Background and history including previous reviews $\ldots \ldots \ldots \ldots \ldots \ldots$

2. Approaches to the study of electronically nonadiabatic molecule-surface interactions . 173

\footnotetext{
${ }^{*}$ Corresponding author. Tel.: +1 805893 8085; fax: +1 8058934120.

E-mail address: wodtke@chem.ucsb.edu (A.M. Wodtke).
} 
2.1. Infrared linewidths . . . . . . . . . . . . . . . . . . . . . 173

2.2. Direct measurement of vibrational lifetimes . . . . . . . . . . . . . . . . 174

2.3. Electronic friction theory and the Newns-Anderson model . . . . . . . . . 176

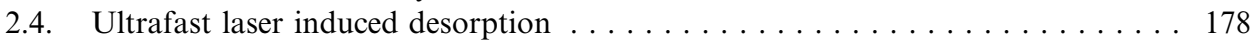

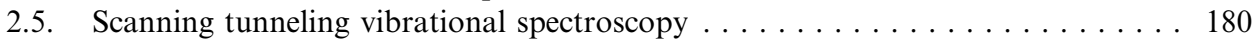

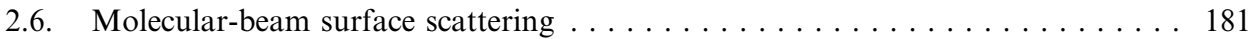

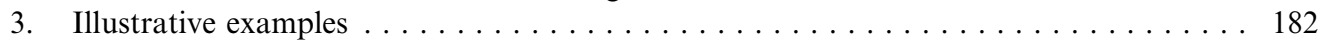

3.1. Comparing electronically adiabatic to nonadiabatic vibrational energy transfer. . 182

3.2. Predicting the magnitude of electronically nonadiabatic effects . . . . . . . . 184

3.3. Observing the transition from adiabatic to nonadiabatic behavior . . . . . . . 186

3.4. Charge transfer in the case of large amplitude vibrational motion . . . . . . . . . 191

3.5. Chemical hole diving in exoelectron emission. . . . . . . . . . . . . . . . . 193

3.6. Chemi-currents measured with Schottky diode sensors . . . . . . . . . . . . . . . 194

3.7. The importance of a spin-transition in chemi-current production . . . . . . . . . 195

3.8. The importance of spin conservation in surface chemical reactions . . . . . . . 197

4. Implications for our way forward . . . . . . . . . . . . . . . . . . . . . . . . . . . 199

Acknowledgements . . . . . . . . . . . . . . . . . . . . . . 200

References . . . . . . . . . . . . . . . . . . . . . . . . . . . . . . 200

"...Motion is the very essence of what has hitherto been called matter." - Lord Kelvin

\section{Background and history including previous reviews}

In championing the "dynamic theory of heat" being researched by Joule in 1872 , William Thomson (Lord Kelvin - Fig. 1) admitted that prior to 1847: "I did not...know that motion is the very essence of what has hitherto been called matter". Joule's work on the mechanical equivalent of heat and the scientific movement he belonged to radically changed our view of the interconnected scientific concepts of heat [1], energy and motion [2], from a material theory concerning the flow of a hypothetical "caloric" fluid to a dynamical view subject to the laws of mechanics. With the acceptance of descriptions of heat as 'jiggling atoms', one may find the rumblings of notions that are now at the heart of the field that would eventually become known as molecular energy transfer.

Indeed, we may trace back the origins of this field even further. While Thomson's blessing of the dynamic theory of heat in the second half of the 19th century was a crucial step in its acceptance by the general scientific community - he was knighted in 1866 and was to become president of the influential Royal Society in 1890 - it was the Dutch-born mathematician Daniel Bernoulli more than 100 years prior (Fig. 2), who clearly formulated an early version of the kinetic theory of gases, and laid down the idea of heat in terms of the motion of atoms [3]. Essential to his contributions, was an analytic derivation of Boyle's empirical law (at constant $T, P V=$ const.) based on the insight that pressure on a surface is produced by the collisions of molecules against the walls of its container. This may be the first and is certainly one of the most profoundly important instances of scientific analysis of the interactions of atoms and molecules at solid surfaces, the topic of this review. 


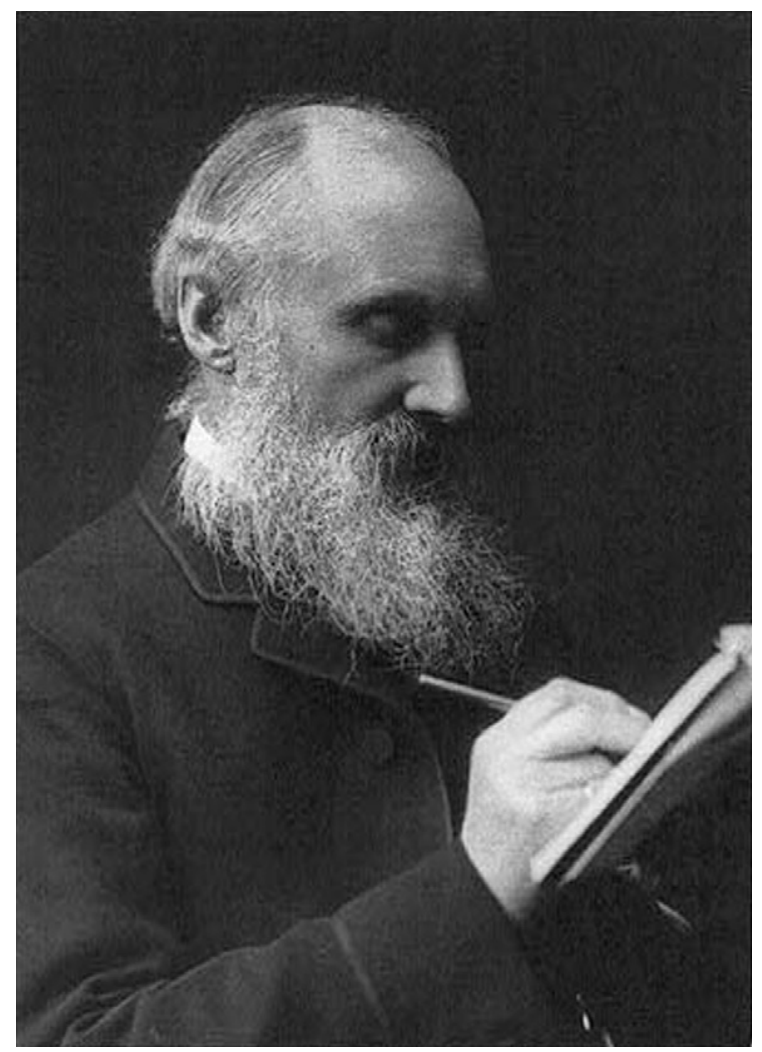

Fig. 1. "...Motion is the very essence of what has hitherto been called matter." - Lord Kelvin.

Today, we are able to study 'the nature of heat transfer' from a dynamically atomistic point of view using tools that Bernoulli, Joule and Thomson could never have dreamed of - opening our mind's eye to view the world of 'caloric motion' with an astonishing level of detail and precision. Observations of macroscopic phenomena are now routinely cast in terms of explanations of microscopic motion and indeed, it is the Bernoullian ambition of obtaining an understanding of the atomistic mechanics that animates the spirit of the field to this day, even when our ideas of (quantum) mechanics have likewise been radically altered.

Irving Langmuir, the first industrial researcher to win the Nobel Prize, helped to establish the technological importance of surface chemistry; certainly, providing much additional motivation to the field of molecular energy transfer at surfaces during the last 100 years. See Fig. 3. Indeed, an atomistic understanding of energy transfer at surfaces is essential to the intellectual infrastructure needed to support the study of such processes as: sticking, diffusion, dissociation, desorption - in short, all of the elementary steps needed to describe surface chemistry.

This writing finds research on energy transfer dynamics at interfaces extraordinarily rich and diverse. A conservative estimate suggests about 100 papers per year are now being published in this field in major internationally recognized journals. Indeed the task of reviewing the field is a daunting one; the development of the field is rapid with new 


\section{DANIELIS BERNOULLI. Joh, Frı, MAY 11882} MED. PROF, Basil.

ACAD. SCIENT. IMPER. PETROPOLITANE, PRIUS MATHESEOS SUBLIMIORIS PROF. ORD. NUNC MEMBRI ET PROF. HONOR.

\section{HYDRODYNAMICA,}

S IVE

DE VIRIBUS ET MOTIBUS FLUIDORUM COMMENT ARII. OPUS ACADEMICUM

AB AUCTORE, DUM PETROPOLI AGERET, CONGESTUM,

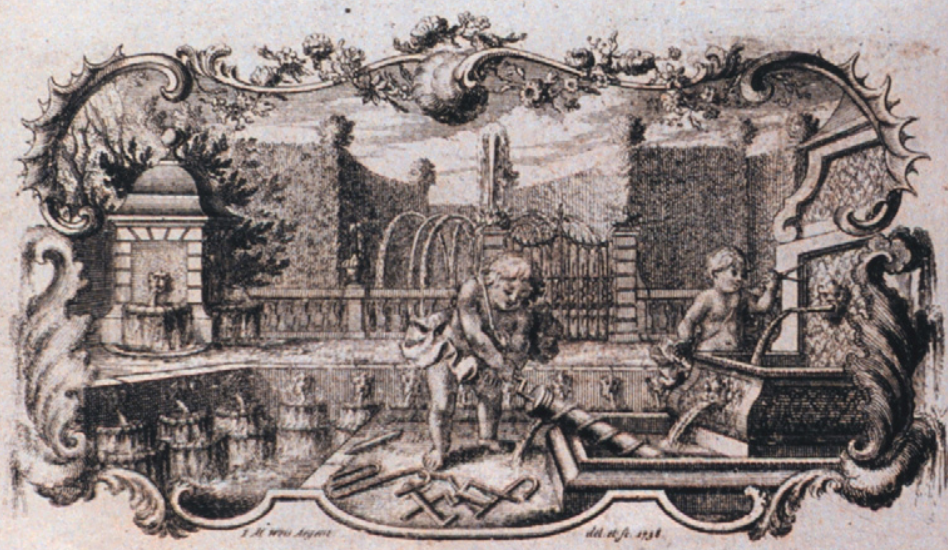

unve

\section{AR GENTORATI, \\ Sumptibus JOHANNIS REINHOLDI DULSECKERI; Anno M D CCXXXVIIl. 1738}

Typis Joh. HeNr. Decker, Typographi Bafilienfis.

Fig. 2. The title page of Daniel Bernoulli's hydrodynamica, an early discussion of energy transfer at surfaces.

problems being defined even as others are, to varying degrees, resolved. Modern topics within this field include:

- State-to-state beam-surface scattering.

- Electron mediated energy transfer.

- Collision or reaction induced electron- and/or photo-emission.

- Tunneling electron induced motion of adsorbates.

- Electronic sensors of chemical energy. 


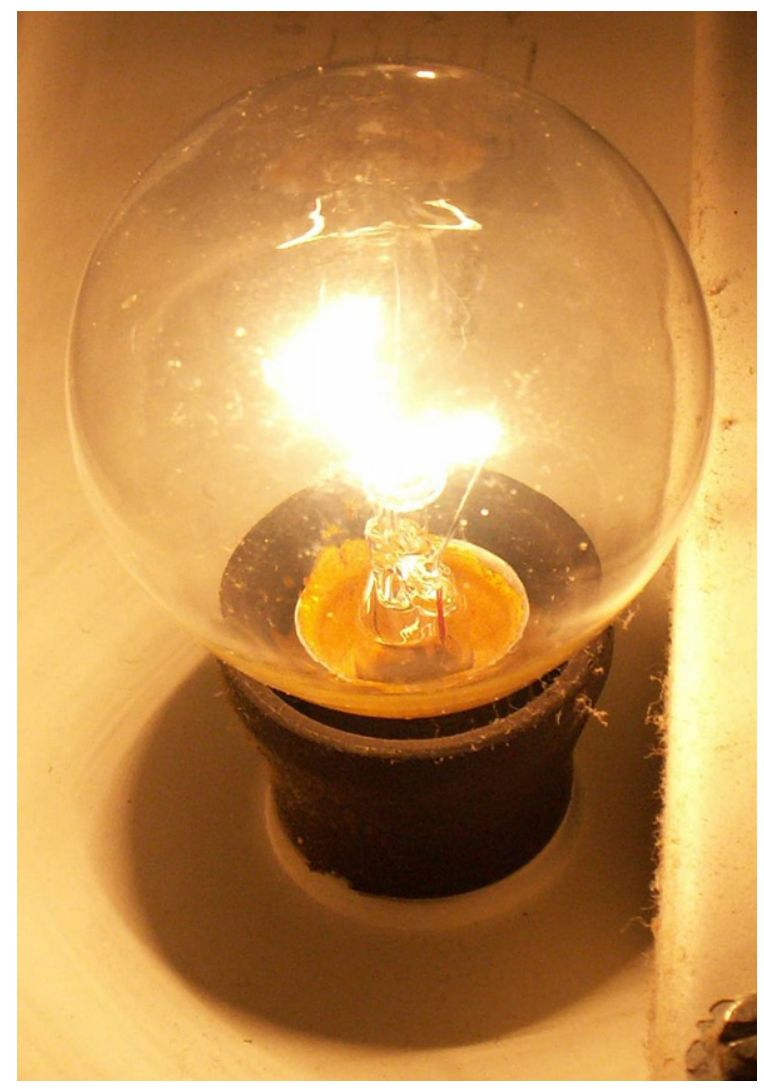

Fig. 3. Let there be light - Irving Langmuir's deep atomistic understanding of surface dynamics enabled him to perfect the incandescent light bulb. By reversing Edison's thinking of seeking an improved vacuum, Langmuir filled the bulb with $\mathrm{N}_{2}$, solving the infamous 'blackening problem'.

- Vibrational relaxation of adsorbates.

- Gas/liquid-surface scattering.

- Molecular interactions with self-assembled monolayers and other soft-surfaces.

- Laser driven surface photochemistry and photo-desorption.

- Ion scattering from surfaces.

To name only some of the most obvious. One might ask how such diversity might be put into proper context. One approach might emphasize those aspects which tend to make energy transfer at surfaces unique; that is, distinct from energy transfer in gas-phase collisional encounters. Such an approach might emphasize the unique energy baths characteristic of solids, specifically, phonons and electron-hole pairs (EHPs). Whereas energy transfer between small molecules is reasonably described by quantum dynamics within the Born-Oppenheimer approximation, phonons may often be treated with classical mechanics. The energy exchange to and from EHPs, however, represents a fundamental breakdown of the Born-Oppenheimer approximation.

Conversely, an alternative unifying context might explore the extent to which the concepts of molecular interactions in gas-phase bimolecular collisions can be extended 
to elucidate molecular interactions at surfaces. In practice, this pathway is being explored by many scientists in this field, if for no other reason than the tools, especially the theoretical ones, needed to implement this strategy are readily available. For example, electronic structure calculations, especially using density functional theory, predict reasonably accurate interaction potentials, especially when periodic boundary conditions can be applied, such as for molecules interacting with a metal surface. See Fig. 4 which depicts work from Ref. [4]. Such calculations are typically carried out by freezing all of the atoms in the solid surface (neglect of phonons). Moreover, DFT being a ground state theory neglects excited EHPs. Such theoretical methodologies have advanced to the point where fully quantum mechanical calculations in up to six dimensions can be carried out and the first 7D calculations are to be expected soon. Mixed quantum-classical dynamical simulations provide avenues for extension to even more degrees of freedom.

As we shall see, a recurring theme of research in this field concerns observations of the special role of electron mediation in energy transfer in molecule-surface encounters. The availability of low energy electronic excitations, especially when the solid is a metal or low band gap semiconductor, makes such behavior common and easy to observe. This places severe demands on theory, especially for the commonly employed DFT. First of all, theoretical treatments beyond DFT are necessary for calculating excited electronic properties. More advanced theories such as the time-dependent DFT and the GW can be applied to calculate the excited electronic states of solids, but are still in an early stage of development and require much larger computational resources [5-7]. Furthermore, while its success in surface chemistry is indisputable, there are certain classes of chemical processes that are notoriously badly described by DFT, one of which is charge transfer chemistry. This is a result of several factors most fundamentally though, that electrons undeniably possess particle like behavior, whereas DFT treats them as a fluid. For example, Unless con-

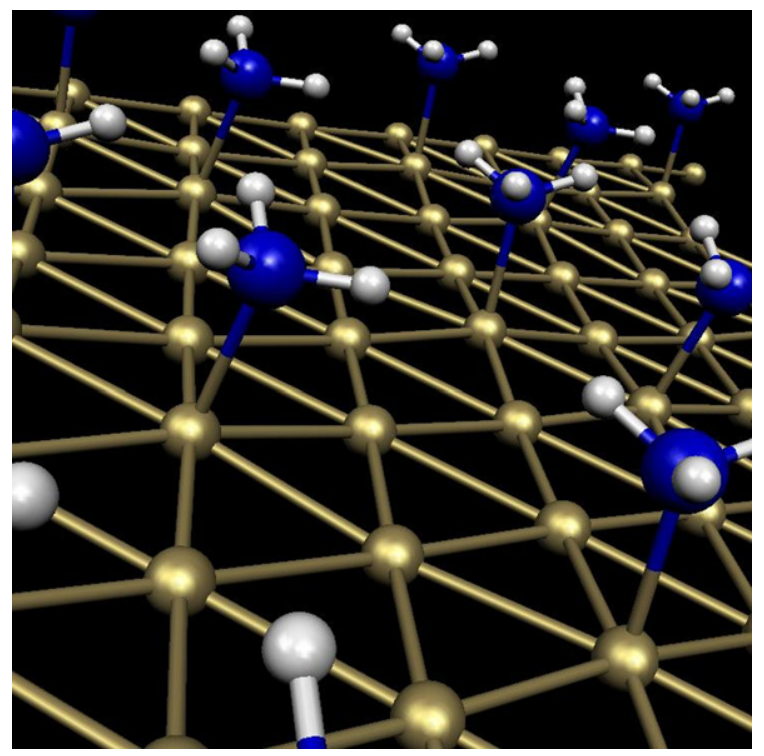

Fig. 4. Periodic boundary conditions allow the calculation of extended systems like this one: $\mathrm{NH}_{3}$ adsorbed on $\mathrm{Ni}(111)$. Only the top layer of $\mathrm{Ni}$ atoms is shown. Image used courtesy of Professor Lauri Halonen University of Helsinki. See Ref. [4]. 
strained, DFT calculation of the dissociation of $\mathrm{H}_{2}^{+}$results in two identical particles, each possessesing half an electron! While DFT will certainly continue to grow in importance for surface chemistry applications and there exist already charge and spin constrained versions [8-11], which seek to overcome these problems. It is clear that additional development within DFT will be needed and indeed, fundamentally different approaches are likely to be adopted before we arrive at a predictive theory of surface chemistry. Thus, while this review will not make a serious attempt to cover the theoretical literature relevant to the title field, it is hoped that the experimental phenomenology covered here will provide inspiration and challenge to the next generation of theoretical chemists.

The plan of this review is as follows. First, we present a section on the background and context of field. This section is a brief overview of selected results designed to provide the reader with a working knowledge of the tools being used by researchers in this field. While this section is less than complete, we hope the value of providing a larger context is valuable. The second section of the article goes into more detail and emphasizes more recent results that have shaped our understanding. We bias this paper toward experimental work and moreover experimental work using molecular beams methods. The authors are all molecular beams experimentalists and have chosen to remain close to home in defining the charter of this paper. Furthermore, undeniably, these methods have and are playing an important role in advancing the field. Nevertheless, most of the experiments described in this review are important because they demand development of new theoretical methods for treating dynamical surface phenomena. In this context, we do take some liberty in providing opinions and speculation about the future of theoretical developments in this field.

Of course, this review is by necessity incomplete. The field of molecular energy transfer at surfaces is remarkably rich and interwoven with many different phenomena. The reader might be understandably dissatisfied with our limited efforts to provide an overview and a context for this vast field. Fortunately, others have provided similar contributions to this one and the reader is referred to that additional review literature not otherwise cited below [12-27].

\section{Approaches to the study of electronically nonadiabatic molecule-surface interactions}

\subsection{Infrared linewidths}

There have been many experimental approaches to the observation of molecular energy transfer at solid surfaces. One of the first employed was infrared spectroscopy, whose application to surface dynamics has been thoroughly reviewed; see for example [28]. These experiments seek to extract the homogeneous line-profiles from an adsorbate's infrared spectrum, obtained for example in absorption, reflection, or by means of a photo-acoustic signal. It is important to recognize that even a carefully prepared surface may be highly inhomogeneous exhibiting binding at terraces, step-edges etc. Furthermore, the sensitivity of IR spectra to the local structural environment makes its inhomogeneous character complex, in principle information-rich, and highly non-trivial. See, for example, the use of high resolution IR spectroscopy to distinguish $\mathrm{Si}-\mathrm{H}$ bonding at terraces versus steps (Fig. 5) and the use of such knowledge to analyze silicon wet-etching [29]. Thus it can be difficult to extract accurate homogeneous line widths from IR spectra of adsorbates. Despite this, infrared line-shapes have provided useful evidence of vibrational coupling of adsorbates to phonons, EHPs and to other adsorbate vibrations [30-35]. For example, Ryberg reported 


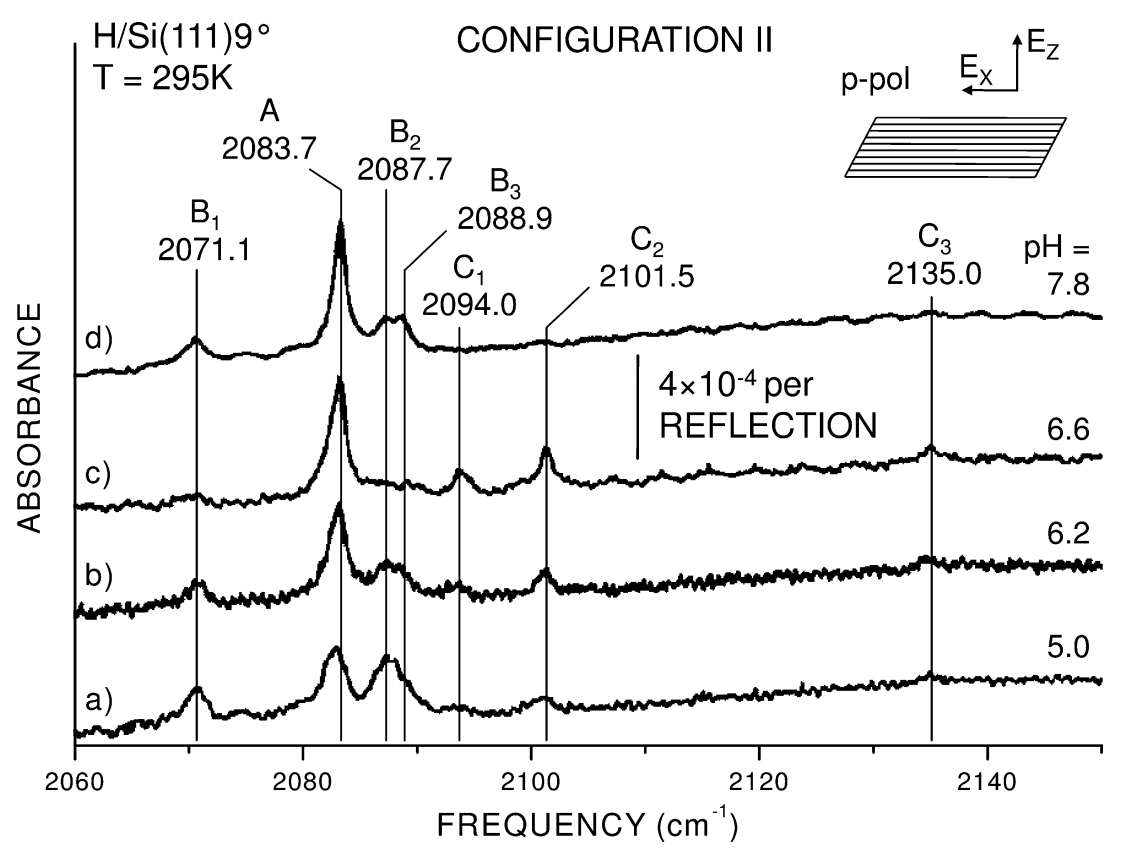

Fig. 5. Infrared absorption spectrum for H-terminated $\mathrm{Si}(111)$ under four (a,b, c, d) different etching conditions. Mode A is identified as a terrace $\mathrm{Si}-\mathrm{H}$ stretch. All others are associated with $\mathrm{S}-\mathrm{H}$ stretches at various stepped defect sites. From Ref. [29].

intrinsic linewidths of $\mathrm{CO}$ on $\mathrm{Cu}(100)$ close to $5 \mathrm{~cm}^{-1}$ suggesting ps timescales for vibrational relaxation [30]. Such large linewidths could not be explained without invoking the vibrational coupling to the electronic motion of the solid [36,37]. Similar studies were also carried out for $\mathrm{CO}$ or $\mathrm{NO}$ on $\mathrm{Pt}$ and $\mathrm{Ni}$ [38-42]. A theory of mechanical coupling of $\mathrm{CO}$ stretch to hindered rotations was found to agree well with experimentally derived lifetimes for $\mathrm{CO}$ on $\mathrm{Ni}$ [38]. While this approach to the analysis of energy transfer at surfaces should not be underestimated, it is no substitute for direct measurements of vibrational lifetimes.

\subsection{Direct measurement of vibrational lifetimes}

Perhaps the first direct measurement of the lifetimes of vibrationally excited adsorbates came from studies of $\mathrm{O}-\mathrm{H}$ (and other) moieties present on high surface-area colloidal silica and zeolites [43-47]. This work showed, for example, $\mathrm{OH}(v=1)$ possessed a lifetime of hundreds of ps, which became substantially shorter when the silica was immersed in common solvents. An important outcome of this work was the clear demonstration that the interpretation of infrared linewidths (i.e. due the difficulties mentioned above concerning distinguishing between homogeneous and inhomogeneous linewidths) can indeed lead to serious errors.

A beautiful early and very clean measurement of adsorbate lifetimes came from infrared emission of $\mathrm{CO}$ from $\mathrm{NaCl}$ (Fig. 6), proving that energy transfer from the high frequency $\mathrm{CO}$ stretch to the low frequency phonons of the solid in this physisorbed system occurs on the ms time scale or slower [48-50]. Also about this time, ultrafast pump-probe experi- 


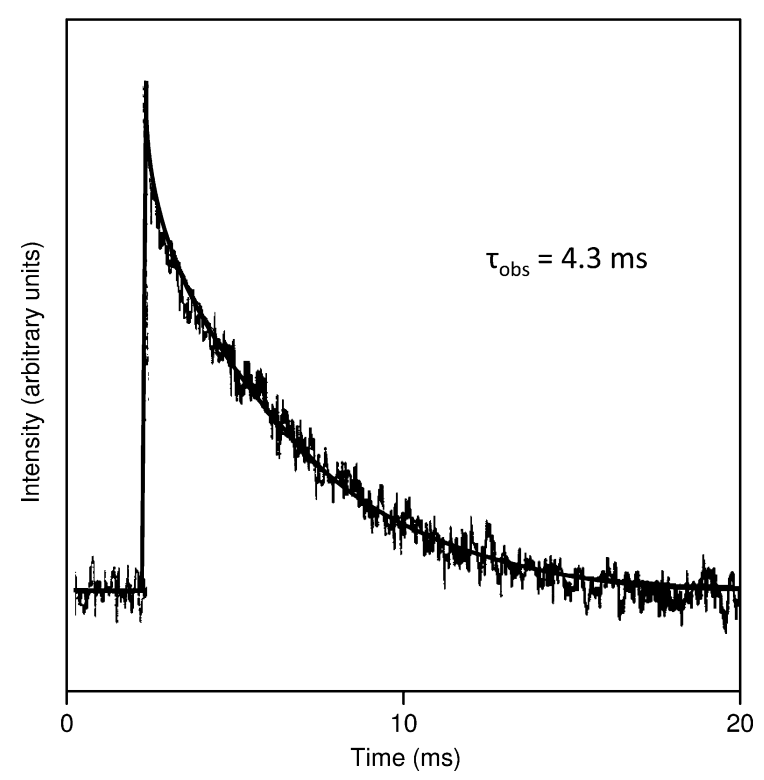

Fig. 6. The infrared emission of $\mathrm{CO}$ adsorbed on $\mathrm{NaCl}$. Coupling of $\mathrm{CO}$ vibration to solid phonons is so inefficient, that the ms timescale emission is easily observed. Adapted from Chang, H.C. and G.E. Ewing, Infrared fluorescence from a monolayer of $\mathrm{CO}$ on $\mathrm{NaCl}(100)$, see [50].

ments became available [51-61], enabling time-domain study of energy transfer of adsorbates on semiconductors and metals. Highlights of this field include experiments showing that $\mathrm{Si}-\mathrm{H}$ vibrations on a $\mathrm{H}$-terminated $\mathrm{Si}(111)$ surface can live for as long as ns [62-65] while $\mathrm{CO}$ vibrational relaxation on copper occurs in only a few ps [66].

A particularly beautiful example of what can be achieved with ultrafast pump-probe technique is the IR-pump/sum-frequency-generation-probe (IR/SFG ultrafast pumpprobe) experiments on H-terminated Si [62-65]. Due to the narrow IR line widths for this surface, it is possible to assign specific spectral features to $\mathrm{Si}-\mathrm{H}$ excitation for $\mathrm{H}$-bonding at terraces and at step-edges, including distinguishing mono-hydride versus di-hydride terminated steps. Furthermore one can selectively pump these surface vibrations. This allowed direct measurements of interadsorbate vibrational energy flow between $\mathrm{Si}-\mathrm{H}$ stretching modes on hydrogen-terminated, stepped vicinal $\mathrm{H} / \mathrm{Si}(1111)$ surfaces using a two-color ps infrared method, in which one vibrational mode is pumped by a resonant infrared pulse and, to observe energy transfer, other vibrational modes are probed by vibrationally resonant SFG. The experiments suggest that interadsorbate energy transfer competes efficiently with slow multi-phonon energy relaxation to the substrate. Relaxation to electronic degrees of freedom is not considered important as the vibrational energy lies in the band gap. Using a kinetic model to analyze the time dependencies, it is found that fast relaxing dihydride-terminated steps (60-120 ps lifetime) drain a large fraction (ca. 2/3) of the terrace $\mathrm{Si}-\mathrm{H}$ mode energy and the terrace mode intrinsic lifetime is derived to ca. 1.4 ns. The model is consistent with terrace-to-step energy transfer by dipole-dipole coupling between $\mathrm{Si}-\mathrm{H}$ oscillators. The relatively slow dissipation of vibrational energy to the solid Si surface may be responsible for recent observations of laser driven surface chemistry on hydrogen terminated Si [67]. Here, an infrared free electron laser was used to drive 
resonant multi-photon excitation of $\mathrm{Si}-\mathrm{H}$ bonds, which resulted in $\mathrm{H}_{2}$ desorption. A strong isotope effect was observed in favor of $\mathrm{H}_{2}$ over $\mathrm{D}_{2}$ which is not seen in thermal programmed desorption. Unfortunately, the mechanism for this observation remains unclear $[68,69]$.

\subsection{Electronic friction theory and the Newns-Anderson model}

In the examples above, the dynamic range of vibrational relaxation lifetimes spans at least nine orders of magnitude from molecules physisorbed on simple insulators (lifetimes in the ms range) to covalent bonding on a semiconductor (ns) to weak bonding at a metal (ps). While it is premature to say we have a full theoretical understanding of vibrational lifetimes (and more generally energy transfer) of adsorbates on solid surfaces, it would be a failing of this review not to point out two particular theoretical approaches that have been remarkably successful: molecular dynamics with electronic friction [15] and the Newns-Anderson theory [70-72].

In molecular dynamics with electronic friction, which is built on a significant body of prior work [73-86], a generalization of classical adiabatic molecular dynamics is made for nuclear motion on a continuum of potential-energy surfaces, meant to describe adsorbate dynamics at a metal surface. In this situation, the Born-Oppenheimer approximation fails, since for any molecular motion - such as vibrations, rotations, or translations - there are resonant electronic excitations of the metal. Such excitations are often highly delocalized, however, so that the continuum of electronic potential-energy surfaces on which nuclear motion occurs are all of similar shape, and can be replaced by a single, effective potential. Nonadiabatic energy exchange between nuclear and electronic degrees of freedom is then represented by frictional and fluctuating forces on the nuclei, and no explicit electronic dynamics are required [87]. It is also a major advantage that the friction coefficients used in this theory can be calculated with ab initio methods; see for example Ref. [88]. Using this approach the vibrational lifetime of the $\mathrm{CO}$ stretch adsorbed to $\mathrm{Cu}(100)$ was calculated to be $\sim 2$ ps with electronic frication and $>1000$ ps without [89]. Other vibrations including the $\mathrm{CO}-\mathrm{M}$ stretch, the $\mathrm{CO}$ frustrated-rotation and the translation were all found to be strongly influenced by electronic frictional dissipation. This work was later extended using friction coefficients calculated from DFT for CO on other metals and for $\mathrm{CN}$ and NO suggesting the generality of electronic frictional dissipation of adsorbate vibration [90]. Good agreement with experiment is found where it is available.

Due to its relatively transparent nature and relative ease of implementation, the friction theory has become the de facto theory of first resort to help interpret experimental findings especially on metals, see for example interpretation of experimental work on the vibrational energy transfer of $\mathrm{H}_{2}$ and $\mathrm{N}_{2}$ to metals [91-93]. The friction theory was also used to evaluate the role of EHP excitation on trapping and surface diffusion for $\mathrm{CO}$ on $\mathrm{Cu}(100)$, concluding that it contributed only about $10 \%$ to the sticking probability and had negligible effect on the diffusion coefficient [94]. An earlier version of a friction like theory suggested that EHP excitation could play a significant role in $\mathrm{H}_{2}$ diffusion on metals $[74,77]$ and in electron transfer at metal electrodes [95]. The friction theory has also found success in modeling ultrafast laser induced diffusion [96] and desorption [97-99].

Despite its success and reasonably wide use, two points are worth noting. First, a much broader effort to apply this theory would be helpful. Up to now, only a handful of surfaces and adsorbates have been analyzed within a friction based approach. It is thus still too early 
to draw firm conclusions. Second, all friction theories employ (in one form or another) a weak coupling approximation and assume interactions with highly delocalized electronic states, which justifies the use of an effective potential. Thus, there will be a class of phenomena that are fundamentally nonadiabatic to which friction theories cannot be applied, namely those with strong coupling and those that interact with localized electronic states. Situations like this appear to include all forms of charge transfer and it would seem that other approaches will be needed for many important cases, see for example Ref. [100].

A second theoretical approach also warrants more detailed exposition due to its frequency of use and comparative success: namely the Newns-Anderson model of molecule surface interactions. The reader is also referred to three reviews in particular that provide more in-depth reading on this subject [70-72]. The so-called Anderson Hamiltonian [101] developed to describe localized magnetic states in metals, was employed first by Newns to describe $\mathrm{H}$-atom bonding to metals [102]. This one-dimensional time-independent model is remarkably successful in capturing many key ideas necessary to understand covalent bonding at surfaces. In this model (Fig. 7), the adsorption is modeled by only three terms: (i) the energy-dependent density of states (DOS) of the adsorbent, $\rho_{0}(\varepsilon)$, (ii) the discrete adsorbate state at an energy $\varepsilon_{\mathrm{a}}$, and (iii) a coupling strength $|V| . \rho_{0}(\varepsilon)$ and $|V|$ may both be modeled simply using analytic functions or derived from electronic structure calculations, for example DFT. Fig. 7 shows how the electronic structure of a single adsorbate orbital is altered by interaction with electronic orbitals of the solid. The surface DOS is shown in grey and the DOS projected onto the adsorbate orbital is shown in blue for many choices of model parameters. Panel (a) shows the dependence on the width of the surface band. Panel (b) shows the changes induced by increasing $|V|$, and (c) represents the changes resulting from an upward energy shift in the solid's electronic band. It is instructive to note that when the interaction strength, $|V|$, is small compared to the bandwidth, the adsorbate represents a broadened resonance within the solid's band. When $|V|$ is large compared to the bandwidth, clearly identifiable bonding and anti-bonding orbitals appear [71].

This simple orbital description of the electronic structure of an adsorbate can also be cast within a semi-classical trajectory approximation - the time-dependent Newns-Anderson model - where $\varepsilon_{\mathrm{a}}$ and $|V|$ become dependent upon the distance to the surface and, consequently, upon time [70,103]. Furthermore, the orbital occupancy can be solved using Keldysh formalism [72,104] or more directly with an "equations of motion" approach [105-107], which is simplified in the so-called "wide band limit" (Fig. 7a - right hand panel). One may, for example, calculate the time-dependent occupancy of the adsorbate's LUMO orbital (e.g. modeled as an affinity level interacting with its image charge). For those cases where LUMO occupancy is finite as $t$ goes to infinity, one obtains a model of ionization in surface collisions [72]. By constructing simple models of how this orbital occupancy influences the molecular structure, one obtains a simple model of electronically nonadiabatic vibrational energy transfer [72,103]. More specifically, as the adsorbate vibrates the electronic resonance being described by the Newns-Anderson approach, shifts up and down in energy around the Fermi level, exhibiting a periodic change of occupancy. This electronic response is not instantaneous and gives rise to a damping of the vibrational motion by electron-hole pair excitations. A particularly remarkable example of success of the Newns-Anderson model is an explanation of the large difference in vibrational lifetimes of $\mathrm{CO}$ versus $\mathrm{CN}$ adsorbed on various metals, which was attributed to differences in the projected density of states at the Fermi level of the adsorbate's LUMO resonance [108]. It has also been shown how the time-dependent Newns-Anderson model may be 

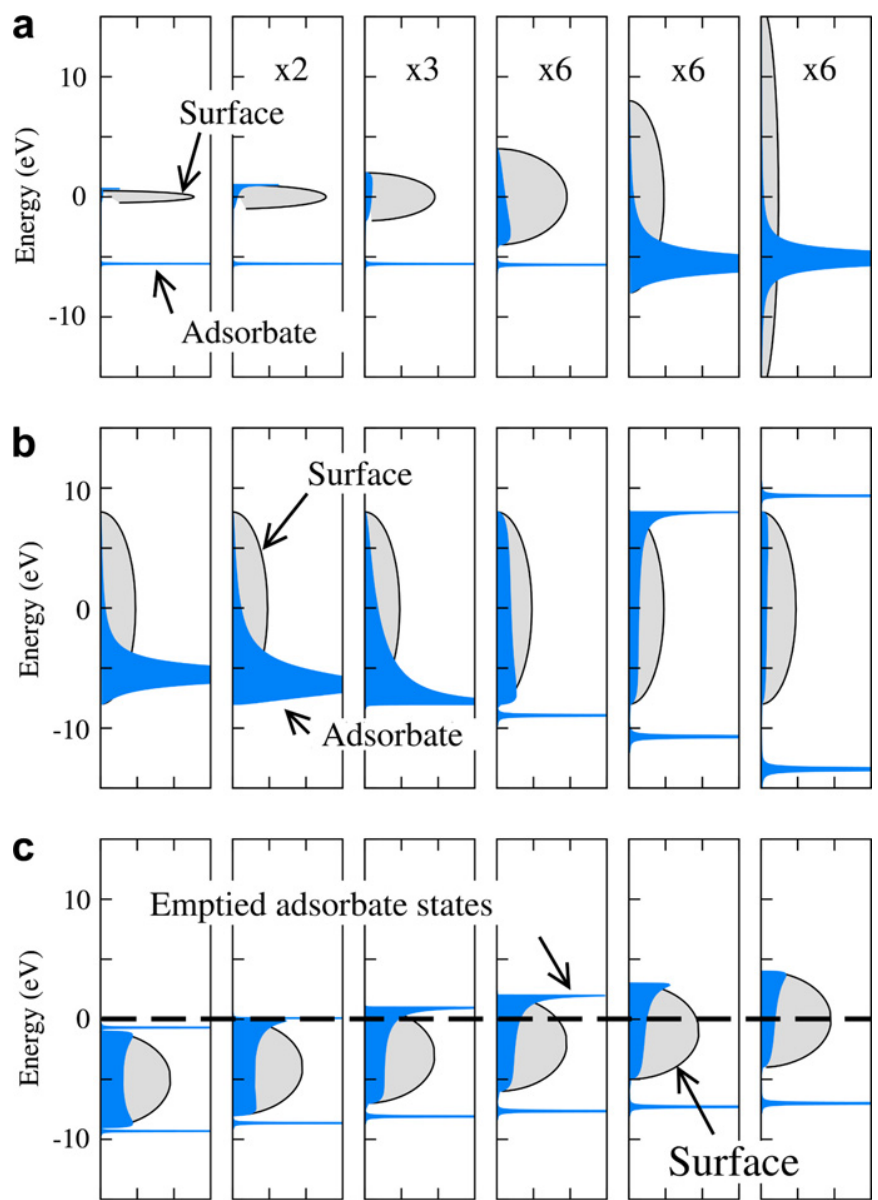

Electronic density of states (DOS)

Fig. 7. The Newns-Anderson model of interaction in an adsorption system with a semi-elliptic DOS band (gray) at the surface and a delta function DOS on the adsorbate before the interaction. The resulting adsorbate state projected DOS (blue) takes a number of forms depending on model parameters. (a) Increasing the bandwidth, $W$. (b) Increasing the interaction strength, $|V|$. (c) Increasing the energy splitting, $\left|\varepsilon_{d}-\varepsilon_{\mathrm{a}}\right|$. The dashed line in (c) is the Fermi level. From Ref. [71]. (For interpretation of the references in color in this figure legend, the reader is referred to the web version of this article.)

modified to provide nonadiabatic energy transfer rates, describing the electron hole pair excitation spectrum [109-111], a topic to which we will return below.

While the Newns-Anderson model is remarkably simple and therefore open to improvements, due to its transparency, flexibility and its ability to lead to intuitive interpretation it has withstood the test of time.

\subsection{Ultrafast laser induced desorption}

The application of ultrafast pulsed lasers, especially with femtosecond (fs) time resolution allowed additional kinds of experiments on the non-thermal laser-driven surface pho- 
tochemistry $[91,99,112-126]$ as well as diffusion [127-130]. These experiments provide a similar degree of insight to that derived from the seminal work on thermal desorption of $\mathrm{H}_{2} / \mathrm{D}_{2}$ from $\mathrm{Cu}(111)$ [131-143], while revealing new dynamics taking place at the sub-nanosecond time scale. For example, as the time delay between two fs laser pulses is varied, one can observe a dramatic (more than linear) dependence on recombinative desorption yield of $\mathrm{H}$ from $\mathrm{Ru}$ when the lasers are nearly overlapped in time [112]. In isotopically mixed (H/D) saturation coverages, a large isotope effect between $\mathrm{H}_{2}$ and $\mathrm{D}_{2}$ desorption is seen [112]. These observations are interpreted as evidence that the laser excited hot substrate electrons mediate the reaction on a timescale that competes with their equilibration with the phonon bath. See Fig. 8. One can also examine the energy content of the desorbed molecules using resonance enhanced multi-photon ionization (REMPI) and time of flight (TOF) methods [113]; the products are found to be translationally and internally excited. For other examples, like $\mathrm{CO}$ desorbing from $\mathrm{Ru}$, the time scale of desorption is much longer (20 ps) and no isotope effect is seen [114]. SFG detection of vibrationally excited $\mathrm{CO}$ in the process of desorbing is also obtained $[115,116]$. Evidence of vibrational energy pooling is also seen $[117,118]$. When one examines the competition between two reactions $\mathrm{CO}_{2}$ versus $\mathrm{CO}$ production from $\mathrm{CO}$ adsorbed to an oxidized $\mathrm{Ru}$ surface, quite interesting results are obtained [119]. Here thermal desorption leads exclusively to CO, whereas fs laser excitation produces $\mathrm{CO}_{2}$. This is taken as evidence of electron mediated $\mathrm{CO}_{2}$ production, similar to the $\mathrm{H}_{2}$ desorption from $\mathrm{Ru}$.

In related work, quantum-state distributions were reported for nitric oxide (NO) molecules desorbed from a $\operatorname{Pd}(111)$ surface at a base temperature of $140 \mathrm{~K}$ by laser pulses of
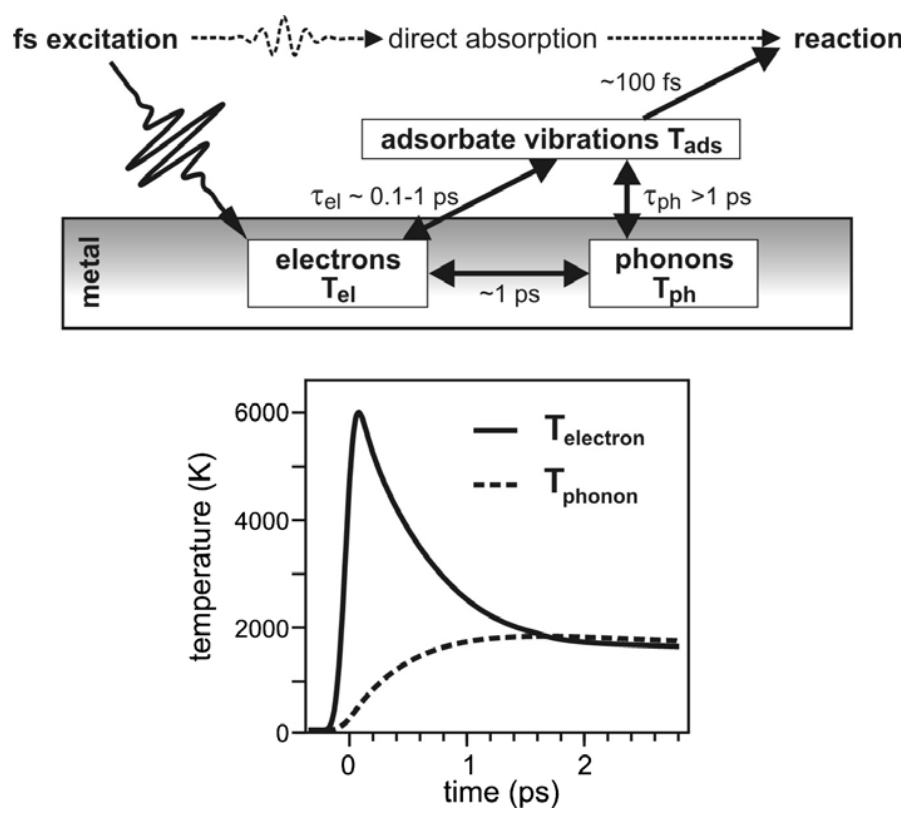

Fig. 8. A schematic diagram showing how laser driven surface chemistry may be markedly non-thermal. Using fs laser pulses, the electrons in the metal can be driven far out of equilibrium with the surface phonons. As electron mediated energy transfer to an adsorbate can be very efficient, this may serve as a means to channel photon energy directly to the reaction center, see Ref. [99]. 
400 fs duration. Significant vibrational populations were observed in the $v=0,1$, and 2 levels. For a fs laser fluence capable of producing substrate electronic temperatures of approximately $4500 \mathrm{~K}$, the vibrational distribution of the desorbed NO molecules is roughly thermal with an average degree of vibrational excitation corresponding to a temperature of $2900 \mathrm{~K}$. The high degree of excitation of the NO intramolecular vibration can be attributed directly to efficient coupling to the EHP generated in the Pd substrate by the fs laser pulse [144].

There has been substantial theoretical work on electronically nonadiabatic photodesorption for which we provide references to recent review articles [145-147], as this topic falls beyond the scope of this review.

\subsection{Scanning tunneling vibrational spectroscopy}

In the experiments just described, a short pulse of laser light, which is absorbed by the substrate results in a transient population of hot electrons that equilibrate with the phonons in several picoseconds. In general terms the molecular desorption process can be considered the result of electron scattering into an adsorbate resonance, inducing adsorbate excitation. One may also examine electron scattering through adsorbates by using the tip of a scanning tunneling microscope, where the tunneling current can induce substrate motion $[148,149]$. See Fig. 9. Indeed, a kind of single molecule vibrational spectroscopy has been demonstrated and exploited [150-166] using an advanced STM instrument [167].

We also mention a few highlights of this work. For example, inelastic electron tunneling spectra for an isolated acetylene $\left(\mathrm{C}_{2} \mathrm{H}_{2}\right)$ molecule adsorbed on the copper (100) surface showed an increase in the tunneling conductance at $358 \mathrm{mV}$, resulting from excitation of the $\mathrm{C}-\mathrm{H}$ stretch mode. An isotopic shift to $266 \mathrm{mV}$ was observed for deuterated acetylene $\left(\mathrm{C}_{2} \mathrm{D}_{2}\right)$. Vibrational microscopy from spatial imaging of the inelastic tunneling channels yielded additional data to further distinguish and characterize the two isotopes [151].

Using this STM induced vibrational excitation, the reversible rotation of a single isolated acetylene molecule between two equivalent sites on the $\mathrm{Cu}(100)$ surface at $8 \mathrm{~K}$ was observed. Excitation of the $\mathrm{C}-\mathrm{H}$ (C-D) stretch mode led to a 10-fold (60-fold) increase in the rotation rate. This increase was attributed to energy transfer from the C-H (C-D) stretch mode to the hindered rotational motion of the molecule [150].

The dynamics and chemistry of individual ethylene molecules adsorbed on the Ni(110) surface at $13 \mathrm{~K}$ have also been studied. By applying a voltage pulse to a single ethylene molecule, the tunneling electrons cause the molecule to reversibly hop away from and back under the tip. A larger voltage pulse (1.1-1.5 V) induces dehydrogenation and the acetylene can be identified through STM vibrational spectroscopy [153].

There is a growing body of work concerning the theoretical interpretation of STM induced vibrational excitation. See for example Refs. [168-173] of which [168,169] are reviews. While this topic is largely beyond the scope of this review, we do note the development of an electronic-friction-like theory designed to interpret the physical origins of the effect [171,172]. Theoretical analysis of $\mathrm{CO}$ vibrational excitation on $\mathrm{Cu}(111)$ and $\mathrm{Cu}(100)$ has been carried out. In this work it is found that the unoccupied $2 \pi^{*}$-orbital on CO turns into a broad and partially filled resonance-like feature in the projected density of states when interacting with the sp and $\mathrm{d}$ bands of $\mathrm{Cu}$. The vibrational damping to the surface of the $\mathrm{CO}$ vibrational motion excited by the tunneling electrons is well described by the Newns-Anderson model for the $2 \pi^{*}$-derived resonance; however, the model is not 

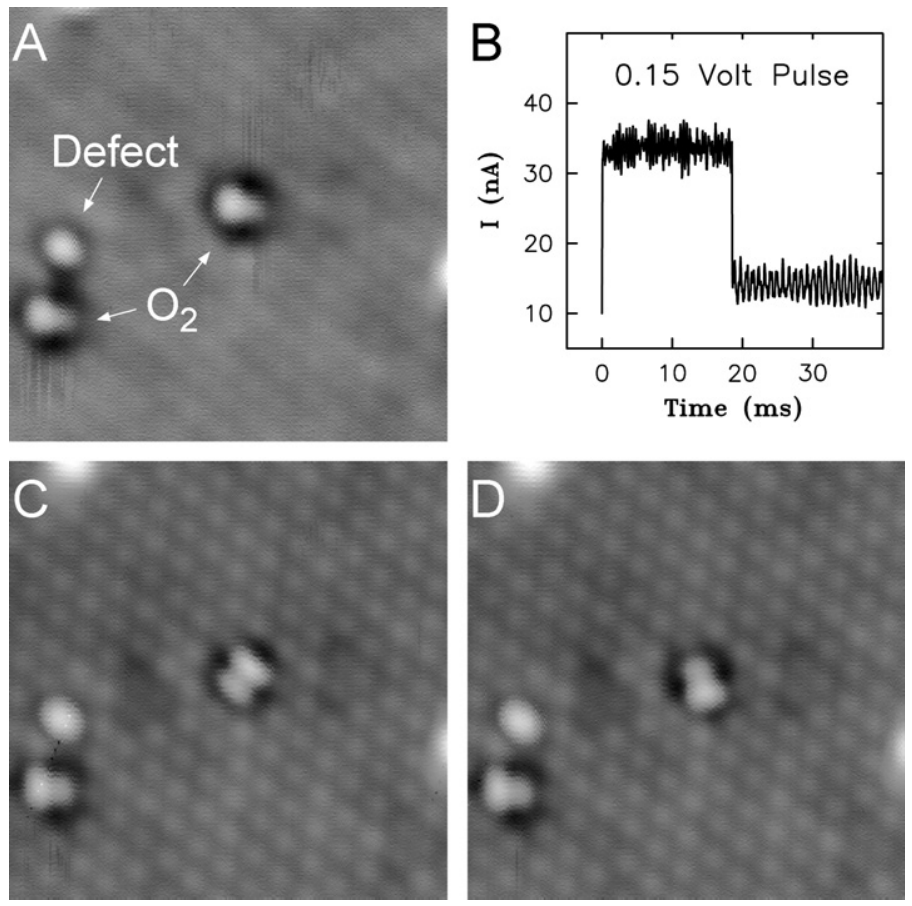

Fig. 9. Observation of the rotation of surface adsorbed $\mathrm{O}_{2}$ due to exposure to electron current tunneling from an STM tip. (A) Prior to the application of the $0.15 \mathrm{~V}$ pulse shown in (B). (B) Time-resolved tunneling current showing the moment of rotation of the $\mathrm{O}_{2}$ molecule. (C) After a single current pulse. (D) After a second current pulse. The $\pi$ clouds of the $\mathrm{O}_{2}$ molecule are visualized (white) in the image. From Ref. [152].

able to describe the nonadiabatic coupling between the tunneling electrons and the vibration. The authors conclude that an important mechanism for vibrational excitation in tunneling is missed by this model; namely, one that involves the change of tunneling amplitude by deformation of the tails of the one-electron wave-functions with vibrational coordinate [172].

\subsection{Molecular-beam surface scattering}

The molecular beam methods as a means to study energy transfer at surfaces have been particularly powerful [174-177]. Molecular beams allow for accurate control of the initial translational energy and direction of the scattering molecules. This approach naturally lends itself to accurate measurement of scattering angular distributions and time-of-flight (TOF) analysis $[178,179]$.

Analysis of scattering angular and TOF distributions and their dependence on initial conditions, for example initial translational energy, can be used to distinguish between trapping/desorption and direct scattering events [180-182] an approach that has recently been extended to atomic and molecular scattering from liquid [183-187] as well as selfassembled monolayer surfaces [188-195]. In similar way, Eley-Rideal reactive events can be immediately distinguished from those that follow a Langmuir-Hinshelwood mechanism [196-202]. 
When the scattering molecule exhibits a Stark effect, hexapoles can be combined with molecular beams to orient the molecule prior to the collision with the surface [203-209]. Laser based detection of scattered molecules can also be used to observe surface collision induced alignments [210-215]. Rainbows in angle-resolved measurements of rotational distributions can be observed [216-222] and in some cases used to extract the orientation averaged depth of molecular physisorption wells [216].

In addition to state-specific laser based detection, quantum-state specific preparation of a molecule prior to its collision with the surface is also possible using molecular beams [223]. For example, infrared excitation of $\mathrm{C}-\mathrm{H}$ stretching motion in methane has lead to characterization of the relative efficacy of vibration versus translation in dissociative adsorption at a nickel surface [224-230]. A similar study for NO $(v)$ excited by narrowband infrared light scattering on $\mathrm{Au}$, provided information on the relative influence of vibration (small) versus translation (large) on trapping [231]. For molecular trapping to an insulator, $\mathrm{HCl}(v=2)$ on $\mathrm{MgO}$, vibrational deactivation is seen to be slow [232]. Stimulated Raman pumping of $\mathrm{H}_{2}$ and its isotopomers is another example of this approach [92,223,233-242].

When combined with state-specific detection, experiments like this can obtain simultaneous control over the initial and final quantum states, translational energies, and incidence and recoil scattering angles. While most work in this direction has been on the scattering of simple diatomic molecules, a few applications of IR absorption and bolometric detection of IR pumped molecules have been reported on systems as complicated as acetylene scattering from $\mathrm{LiF}$ [243-245].

Ion beams are another remarkably useful approach to surface scattering, especially to control the initial and to measure the final translational energies over wide ranges [246]. When combined with resonance enhanced multi-photon ionization (REMPI) ion sources, vibrationally state selected ion-surface scattering is even possible. For example, when NO $(v=0-6)$ was scattered from GaAs surfaces, the vibrational and translational efficacy of $\mathrm{O}^{-}$and $\mathrm{NO}^{-}$production could be examined [247]. The experimental ability to vary the vibrational quantum number of NO in this work is remarkable; however, there are other approaches that, under favorable circumstances work even better, namely stimulated emission pumping [175]. Stimulated emission pumping uses two lasers to excite molecules (typically diatomics) first to an excited electronic state, (with a different bond length) and subsequently de-excite them to high vibrational levels of the ground state. This technique has proven to be very useful in the study of gas-phase energy transfer [174,175,248-262] and is now being applied to study the interactions of highly vibrationally excited molecules with solid surfaces.

In the sections above, we have attempted to provide an overview of the methods being employed in this field, also providing some historical background. We now move to discussion of some key results that have had especially important impact on how we think about these problems.

\section{Illustrative examples}

\subsection{Comparing electronically adiabatic to nonadiabatic vibrational energy transfer}

$\mathrm{NH}_{3}$ colliding with $\mathrm{Au}(111)$ leading to excitation of its umbrella inversion vibration represents one of the most carefully documented examples of mechanical vibrationally 
inelastic surface collisions [263,264]. Fig. 10 shows the vibrational excitation probability's dependence on the incidence energy of translation, $E_{\mathrm{I}}$, exhibiting clear thresholds at the energies where each vibrational channel opens. The relatively low vibrational frequency of this mode $\left(\sim 950 \mathrm{~cm}^{-1}\right)$ couples efficiently with the translation in a surface collision. Indeed, the fact that the thresholds appear precisely where the channels open indicates the importance of scattering events that leave the surface modes more or less as spectators. Furthermore, the excitation probabilities are large, demonstrating that a mechanical coupling of translation to vibration can be efficient. Another important observation in this work was that the vibrational excitation probabilities were nearly perfectly independent of surface temperature, consistent with the idea that surface degrees of freedom are spectators to the vibrational excitation process.

Very different behavior is observed in scattering of $\mathrm{NO}$ from $\mathrm{Ag}(111)$ [265], $\mathrm{Au}(111)$ [266] and $\mathrm{Cu}(111)$ [267]. See Fig. 11. A strong dependence on surface temperature follows an Arrhenius law with an activation energy equal to the vibrational energy gap in NO. The incidence energy dependence also exhibits a "zero-energy threshold" unambiguously showing that the conversion of translational to vibrational energy is not direct. Incidentally, the observed incidence energy dependence was successfully reproduced using the Newns-Anderson model [103]. The Arrhenius surface temperature dependence is predicted from the statistical mechanical population of hot EHP's that may resonantly excite NO vibration, assuming equal excitation probability for all EHP's. This view was challenged by models that successfully reproduced the experimental observations based on thermal phonon to vibration energy transfer [268,269], albeit the activation energy in the Arrhenius form was seen to depend on incidence energy [268,270,271]. Additional theoretical work pointed out that the survival probability of $\mathrm{NO}$ would exhibit much stronger dependence on incidence energy in an electronically nonadiabatic picture than in an

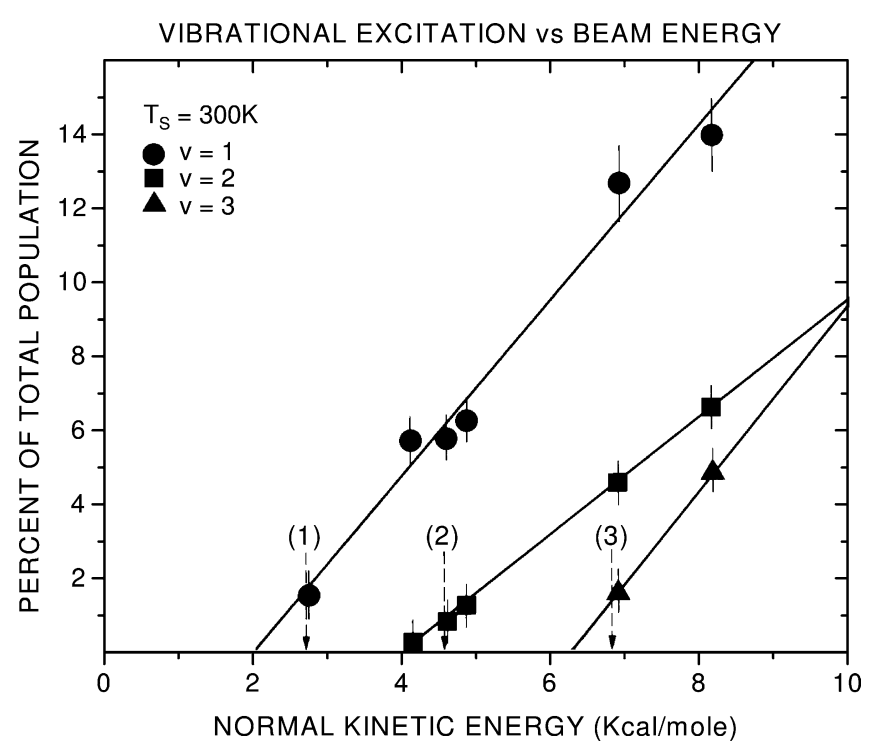

Fig. 10. Adiabatic vibrational energy transfer of $\mathrm{NH}_{3}$ umbrella vibration in collision with $\mathrm{Au}(111)$. The $E_{I}$ dependence exhibits thresholds. From Ref. [264]. 

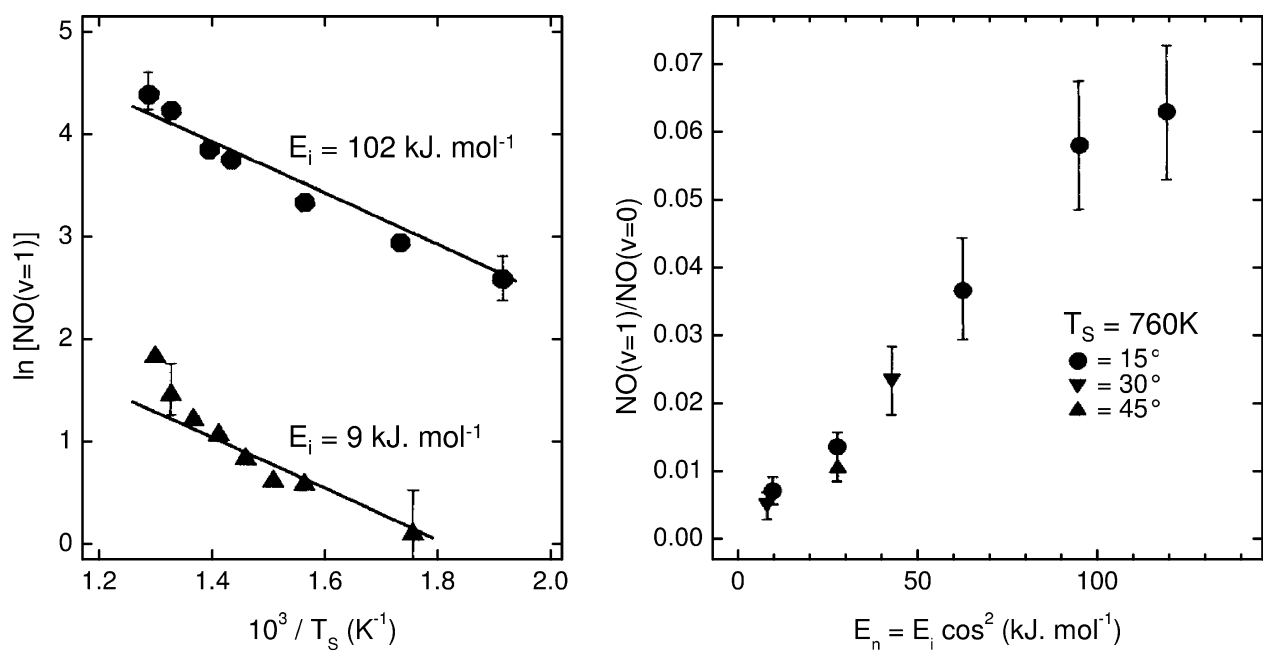

Fig. 11. Electronically nonadiabatic vibrational excitation of NO on $\operatorname{Ag}(111)$. An Arrhenius like temperature dependence and a "zero-energy" threshold in the $E_{I}$ dependence are characteristic. From Ref. [265].

adiabatic one [272]. These insights inspired additional experiments where NO was excited to $v=2$ with a narrowband infrared laser and both excitation and de-excitation could be observed as a function of surface temperature and incidence energy [273], results of which supported the original electronically nonadiabatic interpretation.

\subsection{Predicting the magnitude of electronically nonadiabatic effects}

While EHP-V energy transfer is now reasonably well established for NO/Ag, information and successful connection to first principles theory for a wider variety of molecule/ surface interactions will be necessary before we might gain confidence in being able to predict the magnitude of electronically nonadiabatic interactions. In the absence of such theories, we seek 'intuitive proxies' that might help guide our efforts to seek diversity of behavior in the study of electronically nonadiabatic energy transfer. The energy of the affinity level is one such proxy. Consider Fig. 12, which shows a comparison of three molecules: $\mathrm{NO}, \mathrm{HCl}$ and $\mathrm{H}_{2}[252,274]$. In each case the separated atoms exhibit large electron affinities, which are reduced as the atoms form a chemical bond. When one realizes that the energies of all negative ion states shown in this figure drop dramatically (due to image charge interaction) in the vicinity of a solid surface, it becomes clear that charge transfer may play a vitally important role in the bond dissociation occurring at interfaces. Hellmann has discussed this topic in an extensive way and attempted to accurately characterize how the molecular potentials of several diatomics change with approach to an Al surface $[275,276]$. Here it is enough to point out the qualitative characteristics of the isolated diatomic. Specifically, it is reasonable to expect that energy at which the anion and neutral potential curves cross will influence the degree of vibrationally coupled charge transfer in surface collisions.

Of the three molecules shown in Fig. 12, clearly $\mathrm{H}_{2}$ has the highest energy of anion/neutral crossing. We might therefore expect $\mathrm{H}_{2}$ to behave more adiabatically. This basic ques- 

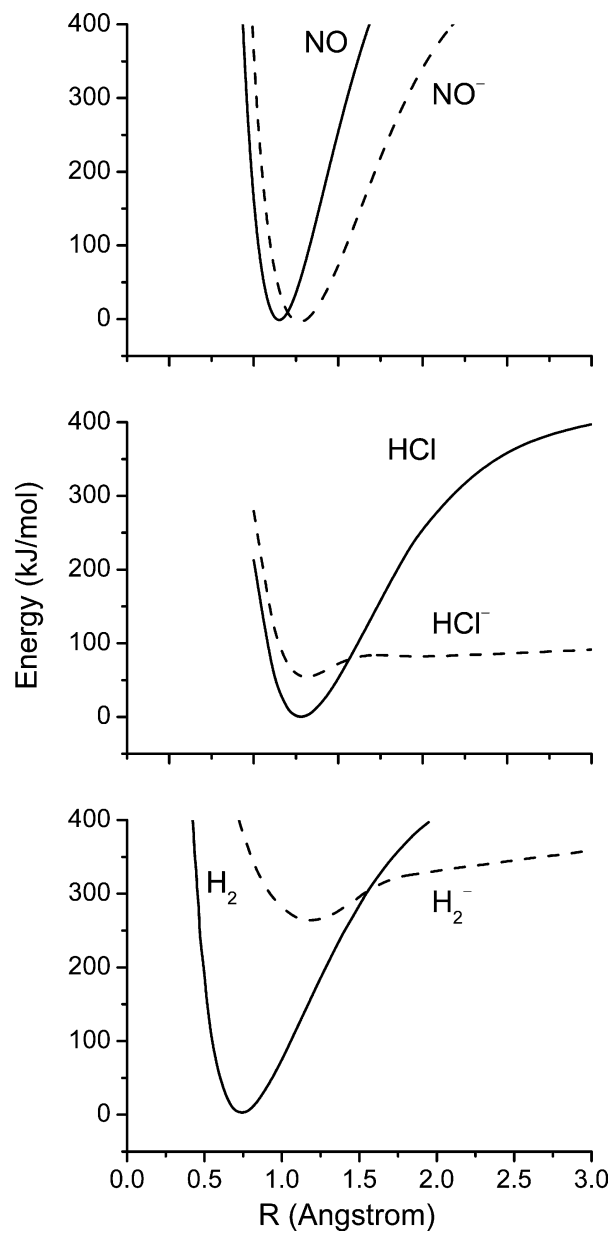

Fig. 12. Comparing the electron binding energetics in three simple molecules. In all cases the electron binding energy depends strongly on bond length. If electron transfer is important in surface interactions, these energetics might play an important role. At present, this represents one commonly employed (but not fully tested) means to attempt to predict electronically nonadiabatic influences. Adapted from Ref. [252,274].

tion has been investigated extensively and is still subject to considerable debate. Translational to vibrational excitation of $\mathrm{H}_{2}$ and $\mathrm{D}_{2}$ in collisions with $\mathrm{Cu}(111)$ were observed with a novel time resolved incidence energy technique [141]. Here a tungsten nozzle with effusive expansion is used with a chopping wheel to create a short pulse of molecules with a wide velocity distribution. Time resolved detection of scattered molecules scans incidence energy. While the excitation probability was large, there was no need to invoke nonadiabatic influences. Rather, the dependence of vibrational excitation on incidence energy was found to resemble the function determined for dissociative chemisorption of molecules in the $v=0$ state [142]. This suggests that translational-vibrational coupling can be strong when molecules closely approach a dissociation barrier. This "failure to launch" mechanism for vibrational excitation in surface collisions is obviously of great importance and furthermore promises to provide one of the very few ways that one might directly probe 


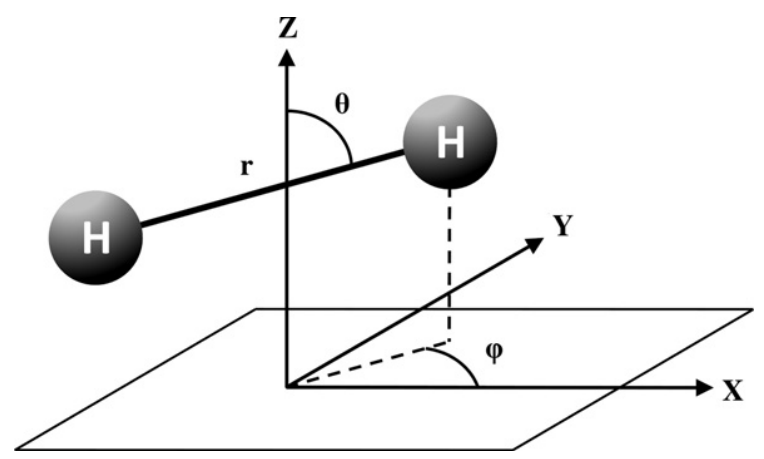

Fig. 13. Six-dimensional calculations of surface chemistry describe distance from the surface $(z)$, bond length $(r)$, orientation of the molecular bond $(\phi, \theta)$, and the site of attack on the surface $(x, y)$. Only with at least sixdimensions, can the site specific nature of surface interactions be properly described.

the nature of the chemical transition state in surface chemistry. Consequently, there has been extensive work on this and related $\mathrm{H}_{2}$ dissociative adsorption systems. The survival probability of $\mathrm{H}_{2}(v=1, J=1)$ in collisions with $\mathrm{Cu}(110)$ was measured directly and found to be $0.74 \pm 0.13$ at incidence energy $=0.078 \mathrm{eV}$ [233] and to decrease with increasing incidence energy [234]. Further work helped to elucidate the loss channels, which include chemisorption and ro-vibrational energy exchange [236,238]. Comparison of extensive experimental data and 6D (Fig. 13) quantum dynamics calculations on a DFT derived potential energy surface have also been reported for this system [238]. Although agreement is good, some significant discrepancies remain, which have recently been attributed to electronically nonadiabatic influences modeled with reduced dimensionality quasiclassical trajectories and ab initio electronic friction coefficients [92].

But these conclusions remain controversial. Indeed, there is more than a little evidence to suggest that the adiabatic approach to surface chemical reactivity using DFT captures much of the physics of the dissociation of $\mathrm{H}_{2}$ on metals [277-279]. The advent of 6D quantum dynamics calculations has proven to be very significant. For example, using a 6D approach with a DFT potential surface gave reasonable agreement with experiments for both $\mathrm{H}_{2}$ dissociative adsorption as well as diffractive reflection on $\operatorname{Pt}(111)$ [280]. Instructively, dissociative reaction probabilities of $\mathrm{N}_{2}$ on $\mathrm{Ru}(0001)$ are dramatically reduced when comparing results calculated with a 2D ("elbow potential") [93] to those obtained in 6D [281]. One reason for this is undoubtedly the importance of site specific reactivity. If, as appears to be the case for $\mathrm{N}_{2}$ in $\mathrm{Ru}(0001)$, only certain surface sites are reactive, the comparison of reduced dimensional calculations to reaction probabilities is highly suspect. On the other hand, calculations of other features of the reaction, for example the vibrational population distribution of $\mathrm{N}_{2}$ from associative desorption may be accurately reproduced with lower dimensional calculations [93].

\subsection{Observing the transition from adiabatic to nonadiabatic behavior}

Referring once again to Fig. 12, the interactions of $\mathrm{HCl}$ with $\mathrm{Au}(111)$ are particularly interesting as it lies intermediate between NO, where much evidence of electronically nonadiabatic effects has been found, and $\mathrm{H}_{2}$ where the role of electronic nonadiabaticity is still 
the subject of considerable debate. Molecular beam scattering of $\mathrm{HCl}$ from $\mathrm{Au}(111)$ was first carried out with state, angle and velocity resolution by Lykke and Kay [282] providing extensive and detailed information on rotational excitation. A schematic diagram of their experiment is shown in Fig. 14, providing a representative example of the instrumentation used in this field. Analyzing the surface temperature and incidence energy dependence of the $\mathrm{HCl}$ rotational excitation, these authors extracted an "orientation averaged" binding energy of $0.2 \mathrm{eV}$, which still serves as the best estimate of the $\mathrm{HCl} / \mathrm{Au}$ binding energy. Angular distribution measurement gave clear evidence of specular scattering and, while it is clear that direct scattering dominates the observations of this work, there is still some question as to the importance of trapping-desorption. Considering the apparatus of Fig. 14, the normal component of incidence energy (as low as $0.02 \mathrm{eV}$ ) is a factor of 10 lower than the incidence energy itself, which was varied from 0.2 to $1.3 \mathrm{eV}$. In light of the derived binding energy one would expect some evidence of trapping. Indeed some reported angular distributions in this work (surface temperature $=300 \mathrm{~K}$, incidence energy $=0.2 \mathrm{eV}$ ) showed tentative evidence of trapping. But at reduced surface temperature the $\cos \theta_{\mathrm{f}}$ contribution disappeared, leading the authors to cast doubt on the conclusion that trapping had been observed. The increased residence time of trapped molecules at $100 \mathrm{~K}$ might provide an alternative explanation for the disappearance of the $\cos \theta_{\mathrm{f}} \operatorname{contri-}$ bution to the angular distribution.

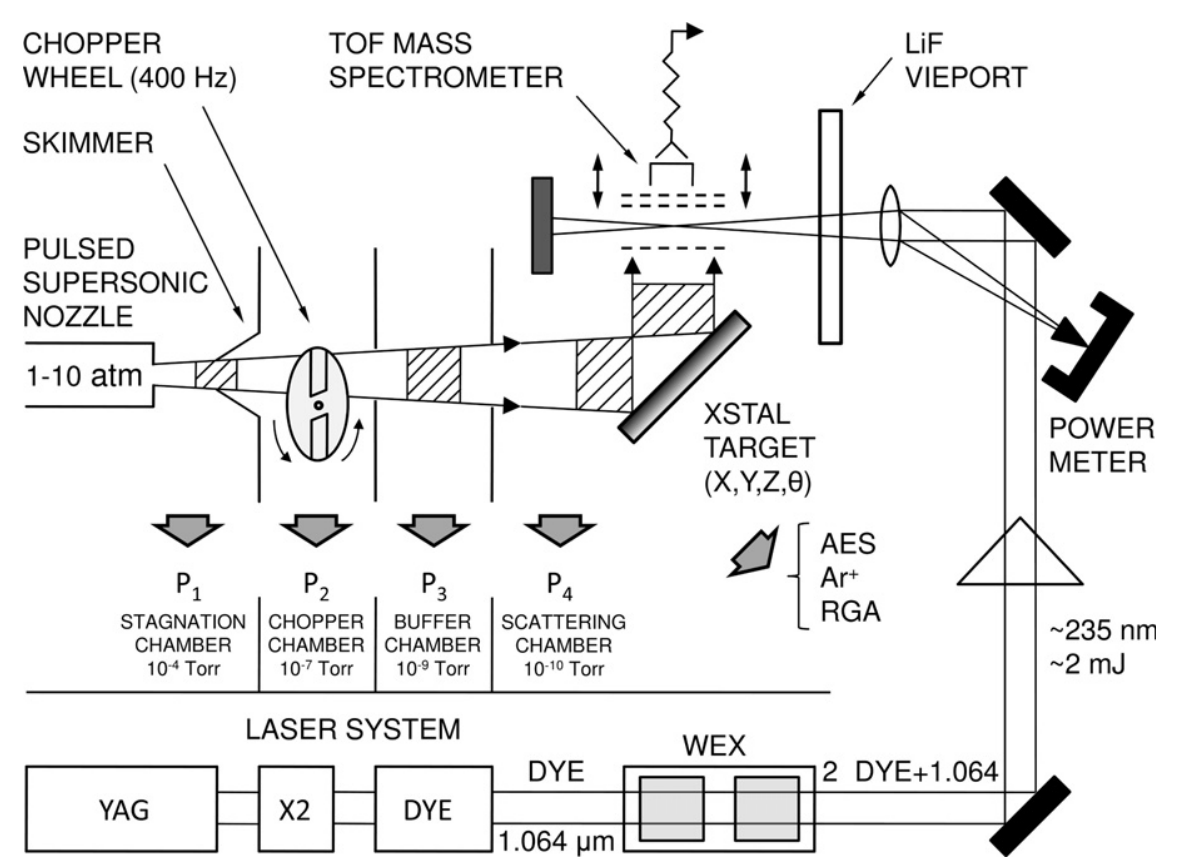

Fig. 14. An excellent example of the experimental arrangement used in surface scattering experiments. A pulsed molecular beam is synchronized with a chopping wheel and laser-based state selective detection is used to monitor scattered molecules. Rotation of the target surface can be used to obtain information on angular distributions. Timing the detection with respect to the chopper provides velocity information. Used by permission from Ref. [282]. 
More recently, this system has been revisited with a new experimental apparatus specifically designed for enhanced sensitivity to low probability scattering events [283]. This apparatus is also capable of obtaining angular distributions at a fixed value of the normal incidence energy, albeit the incidence angle is fixed close to normal. $\mathrm{HCl}$ vibrational, rotational, angular and temporal distributions were recorded for more than 24 choices of incidence energy and surface temperature and extensive data on vibrational excitation of $\mathrm{HCl}$ $v=0-1$ was obtained [284]. See Fig. 15. Several experimental observations suggest a mechanical, i.e. electronically adiabatic mechanism at low surface temperature. First of all, a marked incidence energy threshold is strong evidence that translational energy is directly converted to vibrational excitation. See the discussion of $\mathrm{NH}_{3}$ on $\mathrm{Au}$ above. Less obvious but equally important, the absolute excitation probabilities are some four orders of magnitude lower than for $\mathrm{NO} / \mathrm{Ag}$, perhaps suggesting the efficient electronically nonadiabatic pathway has been "turned off" under these conditions. Interestingly, the observed threshold is substantially above the energy required to excite $\mathrm{HCl}$ from $v=0$ 1. This was rationalized as the inevitable excitation of the surface degrees of freedom in a molecule surface collision. While these results are obviously similar, it is quite interesting to note that the threshold overshoot observed for $\mathrm{HCl}$ on $\mathrm{Au}$, explained as inevitable surface excitation, is not apparent in the case of $\mathrm{NH}_{3}$ on $\mathrm{Au}$. The significance of this is presently not clear.

Additional evidence for a mechanical mechanism at low surface temperature is found in scattering angular distributions, shown in Fig. 16. In each panel the open symbols show the angular distribution of the incident $\mathrm{HCl}(v=0)$ beam. The closed symbols indicate the outgoing scattered $\mathrm{HCl}$ molecules in $v=1, J=6$. The incidence energy of translation is also indicated. The lower panel, which was measured at an incidence energy significantly

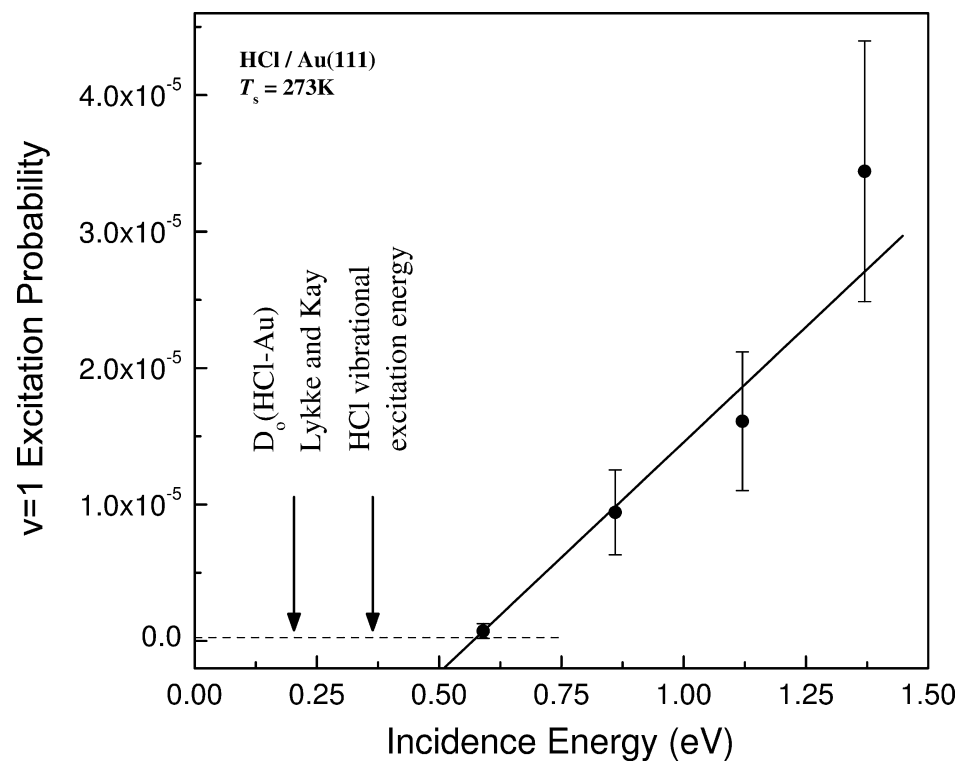

Fig. 15. A mechanical mechanism for vibrational excitation. The vibrational excitation probability of $\mathrm{HCl}$ $(v=0-1)$ in collisions with a room temperature $\mathrm{Au}(111)$ surface. From Ref. [284]. 


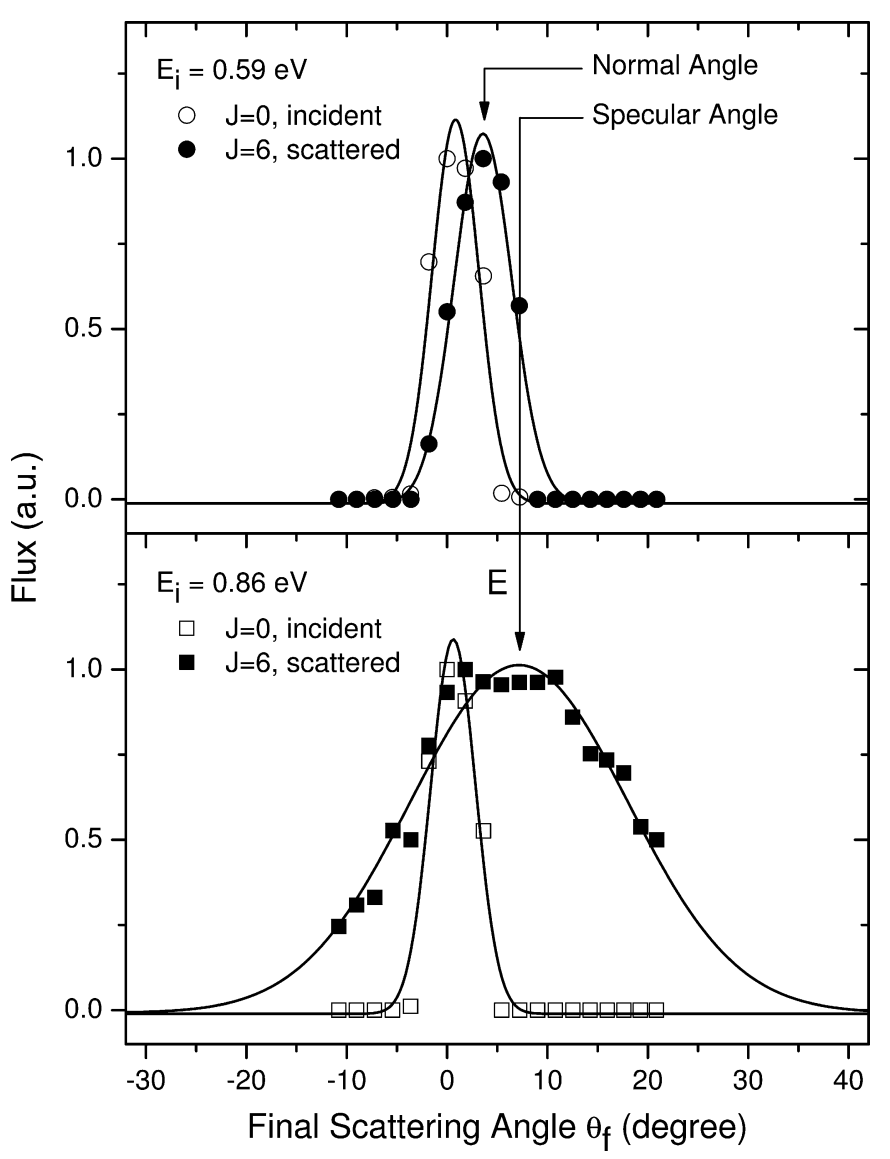

Fig. 16. Further evidence of a mechanical mechanism. The angular distribution of $\mathrm{HCl}(v=1)$ recoiling from $\mathrm{Au}(111)$ collapses and shifts to normal recoil at the threshold (upper panel) for vibrational excitation. The angular distribution above threshold is shown in the lower panel for comparison. From Ref. [284].

above the threshold for production of $\mathrm{HCl}(v=1)$ is representative of most of the experimental observations of this work, exhibiting a $30^{\circ} \mathrm{FWHM}$ and peaking at the specular angle of $7^{\circ}$. The upper panel was obtained very close to the threshold for $\mathrm{HCl}(v=1)$ production. Remarkably, the angular distribution has collapsed to a width limited by the resolutions of the experiments (the angular width of the incident beam) and the peak of the distribution has shifted significantly away from specular in the direction of normal incidence. This remarkable observation was explained by near complete consumption of initial translational energy (by $\mathrm{HCl}$ ro-vibrational and surface excitation) such that only those molecules that happened to depart along the surface normal had the ability to escape the surface. By this explanation, the majority of $\mathrm{HCl}(v=1)$ molecules formed in the collision were trapped to the surface and remain undetected in this experiment.

Perhaps, the most remarkable outcome of this work is the appearance of a change in behavior at elevated surface temperature and reduced incidence energies [285]. This can be seen in Fig. 17, which provides an overview of the results of all of the experiments and a comparison to the previous work for $\mathrm{NO}$ on $\mathrm{Ag}$. The solid lines drawn over the 


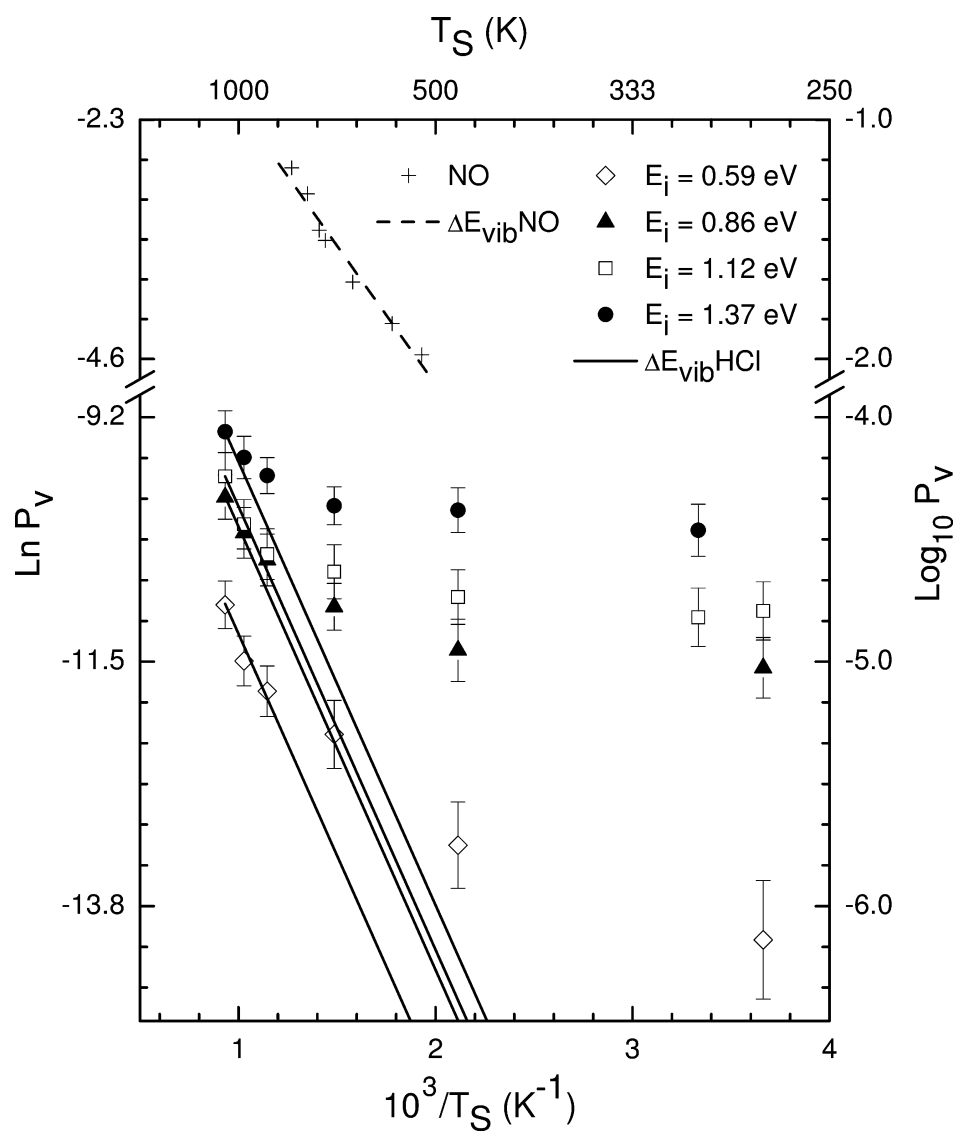

Fig. 17. $T_{\mathrm{S}}$ dependence of vibrational excitation probability of $\mathrm{HCl}(v=0 \rightarrow 1)$ from $\mathrm{Au}(111)$ for incidence energies: $0.59 \mathrm{eV} \diamond, 0.86 \mathrm{eV} \boldsymbol{\Delta}, 1.12 \mathrm{eV} \square$, and $1.37 \mathrm{eV}$. Comparison to NO data is also shown. The Arrhenius like dependence (solid and dashed lines) is expected for hot electron hole pair induced vibrational excitation. From Ref. [285].

derived excitation probabilities are Arrhenius-like plots with the activation energy equal to the vibrational spacing in $\mathrm{HCl}$. This is analogous to the previous analysis for $\mathrm{NO}$ on $\mathrm{Ag}$, where of course the Arrhenius line (dashed) has a smaller slope. These results were interpreted as a transition from a mechanical mechanism to an electronically nonadiabatic mechanism as surface temperature is increased.

Complementary experimental studies examined the reaction $\mathrm{H}_{(\mathrm{g})}+\mathrm{Cl}_{(\mathrm{ad} \text { on Au })} \rightarrow$ $\mathrm{HCl}_{(\mathrm{g})}+\mathrm{Au}(111)$ [200,202]. Molecular beam time-of-flight (TOF) techniques were employed to determine angular and velocity distributions of the $\mathrm{HCl}$ product, and resonance-enhanced multi-photon ionization was used to determine rotational and vibrational state distributions. While evidence of Langmuir-Hinshelwood (LH) reaction, where the $\mathrm{H}$-atom first thermally accommodates to the surface was also seen, the TOF and angular distributions provide clear evidence for an Eley-Rideal (ER) mechanism, that is, direct abstraction of the $\mathrm{Cl}$-atom by the $\mathrm{H}$-atom. This mechanism yields a fast (early) peak in the TOF distributions and a narrow angular distribution that is asymmetric with respect 
to the surface normal. Moreover, the peak in the angular distribution moves further away from the normal in the direction of the specular angle as the energy of the incident $\mathrm{H}$-atom is increased from 0.07 to $0.3 \mathrm{eV}$. The mean energy of the ER product is about $0.6 \mathrm{eV}$, but increases slightly with increasing incidence energy. The fast (ER) TOF component is itself found to be composed of at least two contributions, assigned to $\mathrm{HCl}$ product formed in $v=0$ and $v=1$ (with some contribution from $v=2$ ). The rotational state distribution for the $\mathrm{HCl}(v=0)$ product of the ER mechanism is found to be distinctly non-Boltzmann, with a mean rotational energy of about $0.11 \mathrm{eV}$, or about $5 \%$ of the available energy. The vibrational state distribution for the ER process peaks in $v=1$ and represents only about $14 \%$ of the available energy.

For the ER products, the mean total energy carried away in the $\mathrm{HCl}$ product is only about half of the theoretically available energy and the observed vibrational distribution is much less energetic than a comparable gas-phase reaction $\mathrm{H}+\mathrm{Cl}_{2} \rightarrow \mathrm{HCl}+\mathrm{Cl}$. This suggested that vibrational energy might be efficiently quenched by coupling between the departing molecule and the surface, presumably through an efficient vibration to EHP energy transfer mechanism.

Theoretical treatments using quasi-classical trajectories and a 6D DFT potential energy surface have been used to model the approach of the $\mathrm{H}$-atom to the surface adsorbed $\mathrm{Cl}$ atom. This work also modeled the transfer of energy to the surface phonons using a method developed by Shalashilin et al. [286]. This work identified three possible reaction mechanisms: ER, LH and a "hot atom" (HA) mechanism, where the initially adsorbed Hatom does not fully accommodate to the surface before reaction takes place [287-290]. The calculations showed that the HA mechanism might dominate the reaction, representing an alternative explanation to the low energy content of the products; however, significant discrepancies between experiment and theory remain. For example, experimental observations interpreted as non-reactive $\mathrm{H}$-atom sticking at $100 \mathrm{~K}$ are not found in the calculations, even when the model is implemented with "strong damping". Furthermore, the calculated $\mathrm{HCl}$ vibrational and translational distributions are more energetic than observed. This prompted comment that energy transfer to EHP's might be important in enhancing $\mathrm{H}$-atom sticking (consequently decreasing in importance the HA mechanism) and vibrationally relaxing the departing $\mathrm{HCl}$ molecule [290].

The available energy in the $\mathrm{H}+\mathrm{Cl}_{(\mathrm{ad}}$ on $\left.\mathrm{Au}\right)$ ER reaction is capable of producing $\mathrm{HCl}$ up to $v=8$, which is indeed found in the theoretical calculations. The large amplitude vibrational motion of $\mathrm{HCl}(v=8)$ is highly anharmonic, both mechanically and electronically. Precisely how energy transfer may occur in this case is still an open question; but there is growing evidence that the phenomena observed for $\mathrm{NO}(v=0-1)$ excitation on metals can increase significantly with vibrational energy. Laser assisted associative desorption of $\mathrm{N}_{2}$ from $\mathrm{N}$-atom adsorbed on $\mathrm{Ru}(0001)$ is one such example [93,291]. Here experimentally observed vibrational distributions peak in $v=0$; whereas $2 \mathrm{D}$ theoretical calculations with no coupling to EHP's peak in $v=6$ [93]. Similar results for the vibrational distribution have recently been obtained for adiabatic 6D calculations [292].

\subsection{Charge transfer in the case of large amplitude vibrational motion}

Direct studies of the energy transfer of highly vibrationally excited molecules at surfaces have also been carried out. For example, NO can be prepared in vibrational states higher than $v=18$ using stimulated emission pumping with sufficient efficiency to carry out 
scattering experiments with high signal-to-noise. Vibrational energy loss to metals [252] and insulators [251] is dramatically different. See Fig. 18. On a metal, NO $(v=15)$ looses on average about half of its vibrational energy in a direct scattering collision (sub-picosecond time scale); whereas NO $(v=12)$ looses only a small amount of vibrational energy even when trapping/desorption dominates (pico-nanosecond time scale). While more work is clearly needed, this has been reasonably attributed to efficient vibrational energy transfer to the metal's electronic degrees of freedom.

As an aside, we point out the dearth of similar experimental (or theoretical) work on semiconductors. Here one might predict to see strong and intuitively simple influences of the semiconductor band gap energy on the amount of vibrational energy exchanged with the surface. Perhaps relevant to this is the observation of multi-quantum vibrational relaxation of $\mathrm{NO}(v=15)$ on oxidized copper surfaces [255]. While this work was designed to probe vibrational promotion of copper oxidation [254], it makes plain the feasibility of experiments similar to those presented in Fig. 18 for $\mathrm{Cu}(\mathrm{I})$ and $\mathrm{Cu}(\mathrm{II})$ oxides, which are chemically similar metal semiconductors with very different band gaps.

A possible mechanism, dubbed "vibrational auto-detachment" has been proposed which involves electron transfer from the metal to the NO molecule when the diatom bond is stretched near its outer turning point of vibration and the electron is retransferred to the metal during the anion's bond recompression [248]. Such a mechanism is qualitatively distinct from an electronic friction mediated mechanism where vibrational deactivation energy is coupled to an ensemble of low energy EHP excitations [87]. Here, all of the vibrational energy exchanged with the surface can be transferred to a single metal electron.

Consistent with this idea, experiments carried out on low work function surfaces exhibit vibrationally promoted electron emission [248-250,256]. See Fig. 19. The energetic onset of electron emission is close to the surface work function, strongly suggesting the direct conversion of molecular vibration to the kinetic energy of a single electron.
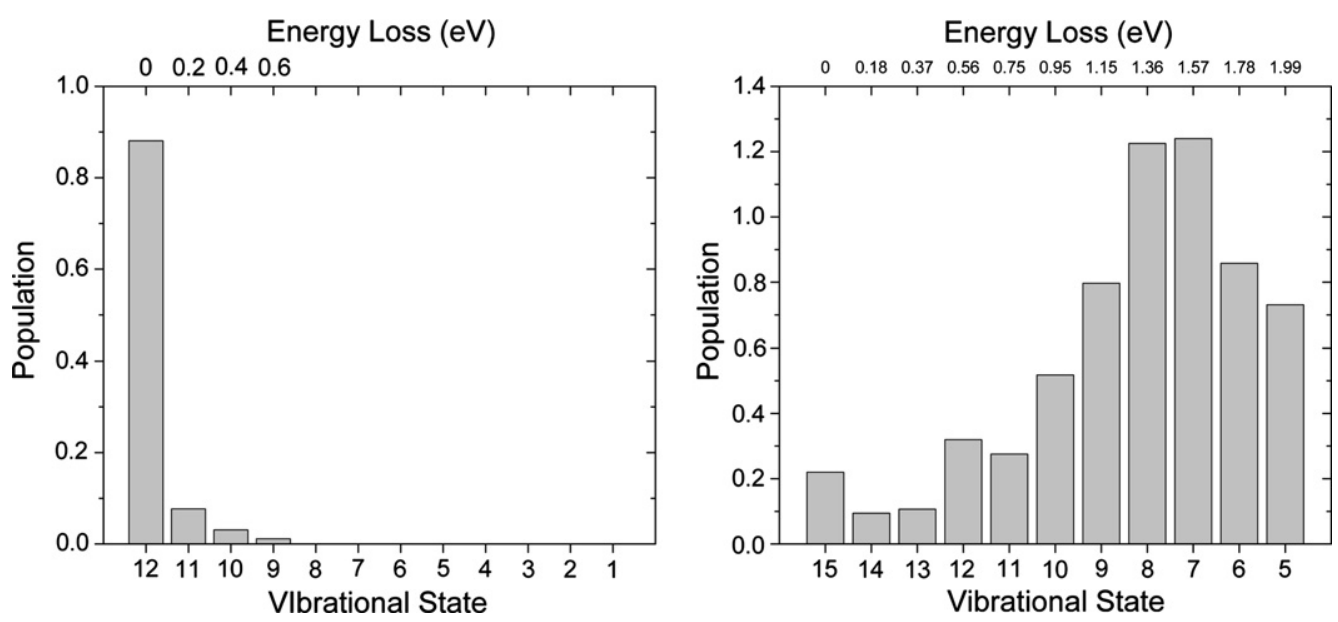

Fig. 18. Vibrational energy loss to an insulator (left) and a metal (right) surface for collisions of highly vibrationally excited NO. Left panel - when NO is prepared in $v=12$, energy transfer to LiF is approximately vibrationally elastic. Right panel - when NO is prepared in $v=15$, collisions with $\mathrm{Au}(111)$ transfer an average of $1.3 \mathrm{eV}$ to the surface. Adapted from Ref. [252]. 


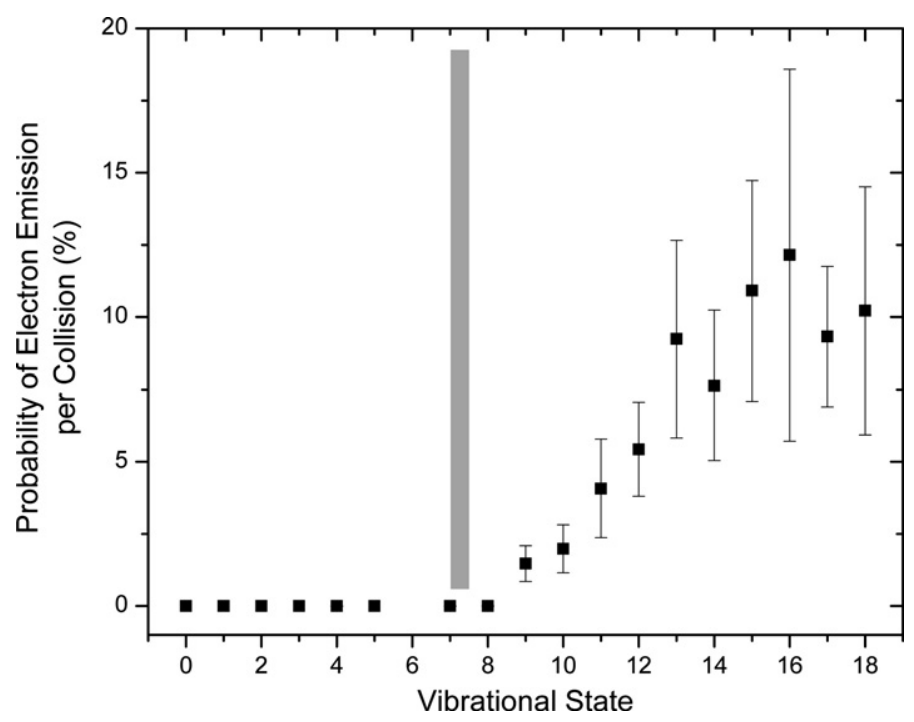

Fig. 19. Vibrational promotion of electron emission resulting from collisions of $\mathrm{NO}(v)$ with a $\mathrm{Au}(111)$ surface with fractional monolayer coverage of Cs. The vertical bar represents the recently determined uncertainty in the work function of the solid. The absolute probabilities have been revised upward from a previous publication, based on more recent work. The figure is adapted from Ref. [250].

\subsection{Chemical hole diving in exoelectron emission}

The topic of chemically induced electron emission from surfaces is, of course, not new and has been reviewed [293-296]. Indeed observation of an analogous process, reaction of $\mathrm{O}_{2}$ with $\mathrm{Al}$ and $\mathrm{Mg}$ producing light, has been known for at least 30 years [297] and less unambiguous evidence for similar processes has been available even earlier [298,299]. A substantial body of observational and mechanistic work is now available on this topic. For example, oxidation by $\mathrm{N}_{2} \mathrm{O}[209,300,301] \mathrm{NO}, \mathrm{NO}_{2}$ [302] and $\mathrm{O}_{2}$ [303-307] of alkali metals results in electron emission. Using $\mathrm{O}_{2}$ as the oxidant in some cases, $\mathrm{O}^{-}$ejection is also seen [307-310]. These observations lead to a novel $\mathrm{O}_{2}$ oxidation mechanism, where sequential two electron transfer from the alkali metal to the $\mathrm{O}_{2}$ molecule ("harpooning") results in an unstable $\mathrm{O}_{2}^{2-}$, which dissociates sending one $\mathrm{O}^{-}$away from the surface and the other toward the surface. The $\mathrm{O}^{-}$heading toward the surface can be considered a hole as it will rapidly be converted to $\mathrm{O}^{2-}$. Due to the low density of states near the Fermi level, this hole dives rapidly below the Fermi level before being filled. Upon filling, an Auger-like process is initiated that can result in electron emission. The term "chemical hole diving" was coined to describe this mechanism [296]. Typical emission probabilities of $10^{-6}$ were observed. For oxidation by $\mathrm{N}_{2} \mathrm{O}$, vibrational promotion and orientation dependence was observed [209,300,301], supporting the importance of harpooning in this chemistry. Critical to this mechanism is the speed of the hole relative to the surface. By increasing the speed of the hole toward the surface using accelerated molecular beams of $\mathrm{O}_{2}$, the electron emission probability increases [311], as the Born-Oppenheimer breakdown becomes more important. The dependence on incidence velocity is in fact quite strong, following a form $P \sim \mathrm{e}^{-v_{0} / v}$ originally proposed by Brako and Nørskov [70,312]. Here, $v$ is the velocity of the incoming molecule and $v_{0}$ is a constant, predicted by the model. 
Returning to the vibrational promotion of electron emission, shown in Fig. 19, this is believed to exhibit a very different mechanism. Specifically a "vibrational auto-detachment mechanism" has been proposed [250]. Here, approximately adiabatic electron capture by NO appears to happen at a critical tunneling distance from the surface when NO is near its outer vibrational turning point. As the transient $\mathrm{NO}^{-}$bond recompresses, the affinity level increases rapidly in energy [252]. In a simple classical view, the relative velocity between the $\mathrm{N}$ and the $\mathrm{O}$-atom reaches about $10,000 \mathrm{~m} / \mathrm{s}$ for $\mathrm{NO}\left(X^{2} \Pi_{1 / 2}, v=18\right)$ within $\tau_{1 / 4} \sim 6 \mathrm{fs}$, shifting the affinity level upward by more than $4 \mathrm{eV}$ (well above the vacuum level) within the first vibrational half-cycle (12 fs). Near the inner turning point, a vertical electronic transition to the neutral state may result in conversion of large amounts of vibrational energy to electron excitation [252]. This mechanism is similar to a recent model [100], which has successfully captured the most important qualitative aspects of the experimental observations.

\subsection{Chemi-currents measured with Schottky diode sensors}

The experiments just described are some of the only ones where the hot electrons produced in Born-Oppenheimer breakdown can be directly observed through emission. But they are not the only ones of this kind. In 1999, some of the most exciting experiments on the topic of electronically nonadiabatic molecule surface interactions were carried out using Schottky diodes to directly detect hot electrons produced by exoergic surface chemical reactions [313-316]. See Fig. 20. Specifically, ultrathin metal films were evaporated on $\mathrm{Si}(111)$ surfaces at substrate temperatures of $175 \mathrm{~K}$. By use of a microfabricated device structure, zero-force electrical contacts were formed on the thin $\mathrm{Cu}$ layers during evaporation. They allowed current/voltage measurements of diodes with $\mathrm{Cu}$ films between 40 and $60 \AA$. The diode formed in this way exhibits a large inhomogeneous interface with a

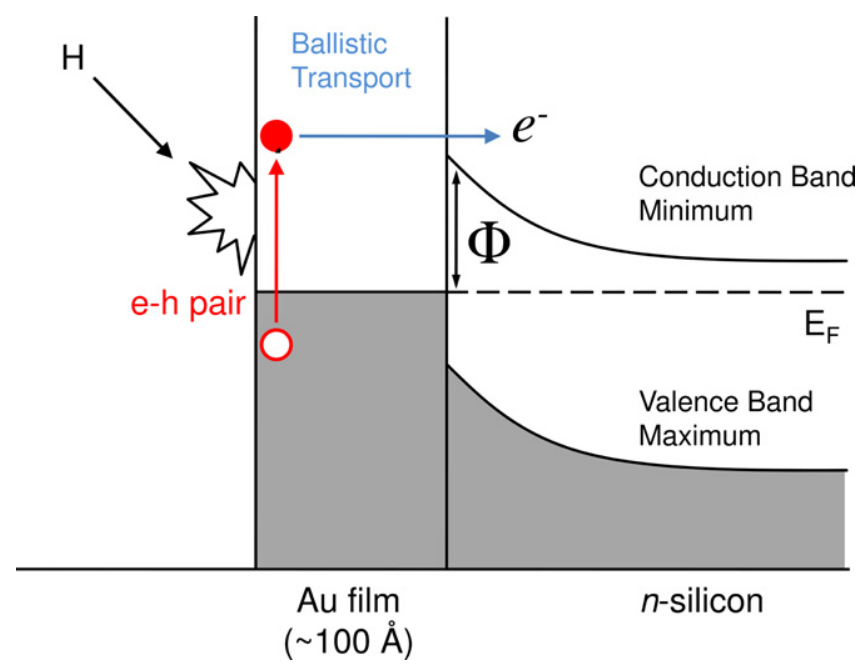

Fig. 20. Schematic diagram of a Schottky diode chemi-current sensor. Exothermic adsorption to a metallic thin film creates hot electrons that are transmitted above the Schottky barrier, $\Phi$. The Fermi level of the metal is indicated as $E_{\mathrm{F}}$. Adapted from Ref. [318]. 
barrier height of $\sim 0.5 \mathrm{eV}$. Remarkably, these thin-metal diodes may be used as atomic hydrogen sensors. The term 'chemi-current' was coined to describe the observed flow of electrons from the $\mathrm{Cu}$ film through the conduction band of the $\mathrm{Si}$ to the collector when the diode is exposed to $\mathrm{H}$-atoms. The current is based on electronically nonadiabatic EHP creation which occurs during exothermic adsorption of hydrogen on $\mathrm{Cu}$ surfaces. This phenomenon has since been demonstrated for a wider variety of surface reactions and replacing silicon by germanium [293,317-326]. In similar devices, others have also observed chemi-current, for example produced by the catalytic oxidation of $\mathrm{CO}$ to $\mathrm{CO}_{2}$ [327-334]. Temperature dependent measurements showed an Arrhenius like behavior to the chemi-current, which had a similar activation energy to the surface reaction [327]. Incidentally, by placing photoreceptors at the thin metal film's surface, a new approach to photovoltaics was demonstrated [335-337].

Up to now there have been very few experiments, which directly detect the energy distribution of the hot electrons produced by energy transfer at surfaces. For exoelectrons resulting from Cs oxidation, low resolution results were obtained using a hemispherical energy analyzer [305]. Interpreted within the chemical hole diving mechanism described above, the maximum energy cut-off suggested that holes could dive up to about $2.5 \mathrm{eV}$ below the Fermi level. Of course, the energy distribution of the endo-electrons is not obtained in such an experiment. More recently, a strong isotope effect between $\mathrm{H}(3.7 \pm 0.7)$ and $\mathrm{D}(1)$ atom chemi-current is seen [324,325]. Assuming a Boltzmann electron energy distribution, this isotopic ratio was used to derive the electron temperature for $\mathrm{H}$ on $\mathrm{Cu}\left(T_{\text {eff, } \mathrm{H}}=1690 \pm 300 \mathrm{~K}\right)$, which compares favorably to that calculated from a novel electronic-friction based model $[88,110,111]$, which we now discuss in more detail.

\subsection{The importance of a spin-transition in chemi-current production}

While other theoretical frameworks have been proposed to understand the electronic excitation spectrum [338], one in particular has met with significant success. This approach starts with a standard first principles electronic structure theory energy calculation of $\mathrm{H}$ at a position above the $\mathrm{Cu}$ surface, and proceeds to combine classical, quantum oscillator, and time-dependent density functional methods to provide a consistent description of the nonadiabatic energy transfer from adsorbate to substrate. An important characteristic artifact of this theory is that the friction coefficient diverges when the DFT calculations are carried out within an assumption of spin adiabaticity; that is, minimizing the total energy of the system with respect to variation of the magnetization density as well as the charge density [111]. More descriptively, the spin adiabatic DFT calculation predicts that as the affinity level of the $\mathrm{H}$-atom passes through the Fermi level, the spin polarization is suddenly quenched [110]. See Fig. 21 - Left panel, which represents the adiabatic limit of the filling of the two spin states of the $\mathrm{H}$ 1s orbital, approaching a copper surface. While the influence of the spin-transition on the potential energy is insignificant, it causes the electronic friction coefficient to diverge.

This spin-transition is an unphysical result predicting infinite stopping power due to electronic friction. It arises from the difficulty of describing spin conservation with DFT in this system. In a somewhat ad hoc way, a spin-constrained version of DFT was applied, which was successful in removing the divergence of the friction at the spin-transition distance, replacing it with a smooth function that peaks at this distance. It is this theory that 

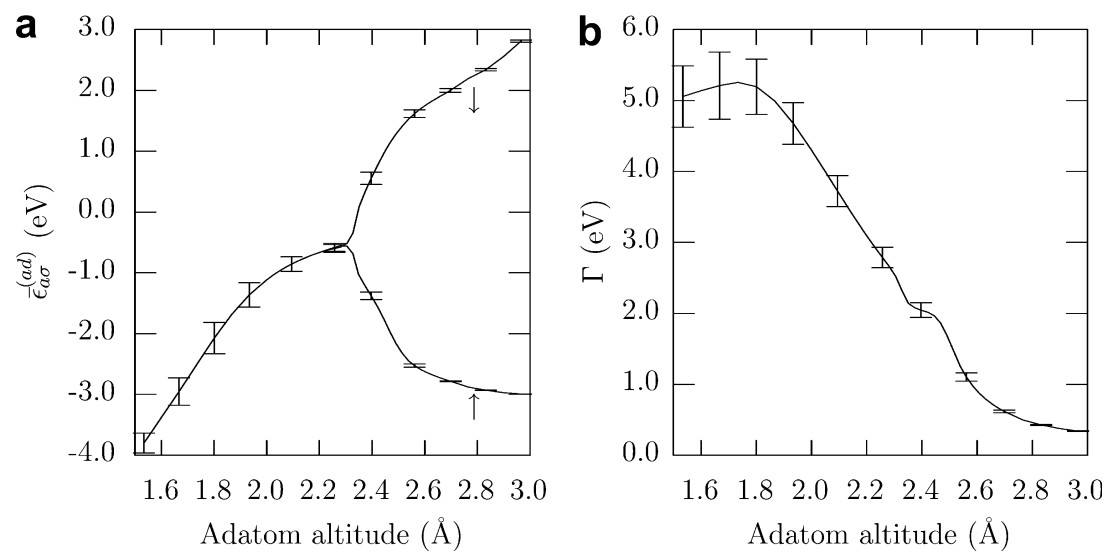

Fig. 21. Spin-transition as $\mathrm{H}$ approaches a copper surface as derived from spin adiabatic DFT calculations. Left panel - the H-atom affinity level (a half-occupied 1s orbital with spin up) suddenly accepts another electron (spin down) at $2.35 \AA$ above the surface, where the affinity level crosses the Fermi energy. Right Panel - the width of the projected density of states onto the 1s orbital (assumed equal for both spin states) can also be calculated. Reproduced from Ref. [107].

Nienhaus used to extract his electronic temperature from the measured isotope effect $[324,325]$.

More recently DFT calculations were used to derive the affinity level's resonance energy and width, two key parameters necessary to employ the Newns-Anderson model of electronically nonadiabatic adsorption $[107,109,339]$. These are shown in Fig. 21. Using these as "input data" to the Newns-Anderson model, nonadiabatic dynamics were calculated (Fig. 22). Here the orbital occupancy of the initially occupied and unoccupied (affinity level) spin states can be seen as a function of time for a trajectory passing through the spin-transition region. Here the solid lines are the adiabatic limit and the dashed lines indicate a typical outcome for realistic nonadiabatic input parameters for $\mathrm{H}$ on $\mathrm{Cu}(111)$. Notice the significant time lag in orbital filling between the adiabatic and nonadiabatic cases. In the right panel, the energy transfer rate, which diverges in the adiabatic limit solid line - is moderate and finite for the nonadiabatic case. One important outcome of this work is the demonstration that electronic friction theories or other "nearly adiabatic theories" are incapable of realistically describing the energy transfer of $\mathrm{H}$-atom translation to EHP excitation near the spin-transition distance [107]. Thus the previous interpretation of the isotope effect to derive an electronic temperature must be viewed with caution [324,325].

Using a fully $a b$ initio approach, time-dependent density functional theory for the electronic degrees of freedom has been combined with Ehrenfest dynamics for the nuclei to simulate electron-hole pair excitation due to electronic friction during the chemisorption of $\mathrm{H}$-atoms on an $\mathrm{Al}(111)$ surface [340-345]. The importance of EHP excitation in the vicinity of the spin-transition is confirmed in these calculations [341] and an isotope effect is found [340]. Due to the computational overhead, this approach has not yet been extensively applied and compared to experiment. Nevertheless, one can calculate the spectrum of electronic excitations for a single trajectory of a few hundred femtoseconds with a single PC. Parallelization of this approach appears quite promising. 

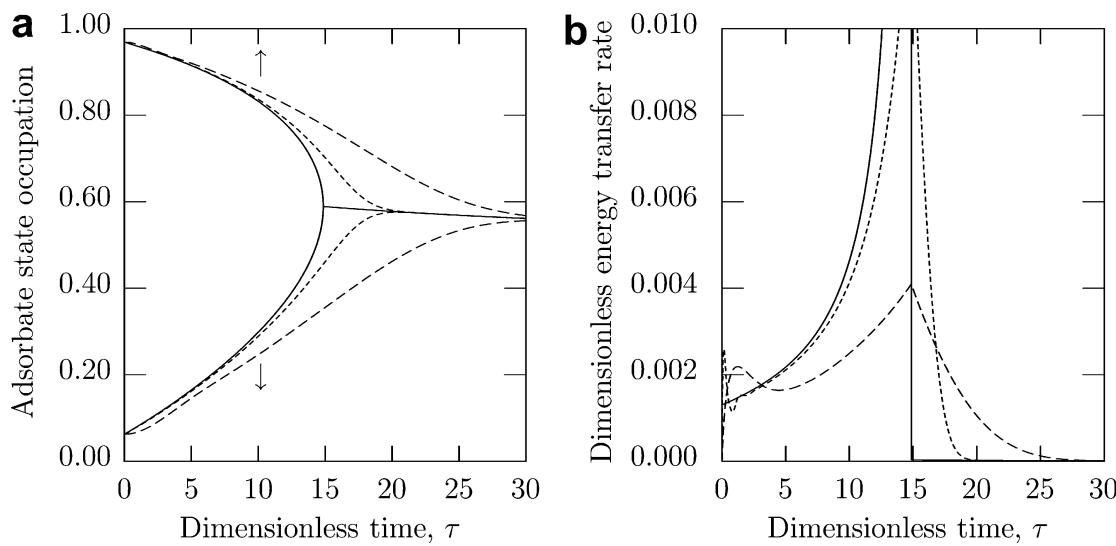

Fig. 22. Application of Newns-Anderson model in the vicinity of the spin-transition as $\mathrm{H}$ adsorbs to copper. As time increases, the H-atom moves toward the surface and through the spin-transition. The left panel shows the occupancy of the two 1s spin states. The right panel shows the energy transfer rate to EHP excitation. The solid line is the adiabatic limit where the energy transfer rate diverges. The dashed line shows a realistic nonadiabatic outcome from the Newns-Anderson model. Note that charge transfer lags as the trajectory passes the adiabatic spin-transition. The dotted line shows a fictitious calculation of a 100-fold slower trajectory approaching the adiabatic limit. The energy transfer rate is reduced as expected. From Ref. [107].

As already mentioned, for the case of H-atom adsorption on $\mathrm{Cu}(111)$ or $\mathrm{Al}(111)$ the potential energy is not significantly influenced by the spin-conservation related problems exhibited by DFT in the above examples. But this may not always be the case.

\subsection{The importance of spin conservation in surface chemical reactions}

The dissociative adsorption of $\mathrm{O}_{2}$ on $\mathrm{Al}\left(\begin{array}{lll}1 & 1 & 1\end{array}\right)$ has attracted substantial attention as it represents a remarkable breakdown of the electronically adiabatic application of density functional theory to properly describe the role of $\mathrm{O}_{2}$ translational energy to promote the reaction. To understand the problem it is simplest to consider the nature of the potential energy surface calculated from spin-unconstrained DFT. A 2D cut through the calculated 6D hyper-surface is shown in Fig. 23 (upper panel), where one would quickly conclude that this reaction appears to proceed without an entrance channel barrier. Yet experimentally (lower panel), when the translational energy is increased the reaction probability increases dramatically, strongly suggesting a barrier along the $z$-coordinate [346]. It is likely that an important step in this reaction (and indeed in many surface reactions) involves electron transfer from the metal to the molecule forming $\mathrm{O}_{2}^{-}$and even $\mathrm{O}_{2}^{2-}$ which leads rapidly to dissociation of the $\mathrm{O}-\mathrm{O}$ bond. As DFT is known to have difficulty describing transfer of single electrons, one should not be entirely surprised that problems arise for this system. Preliminary attempts to more correctly model electron transfer in this and similar reactions have been carried out and point out the importance of taking electron transfer properly into account $[275,276]$. An alternative view of $\mathrm{O}_{2} / \mathrm{Al}$ emphasizes the role of spin selection rules posing the reasonable question: should there not be a triplet and a singlet potential surface? $[8,347]$. Certainly at distances where the molecular orbitals of the $\mathrm{O}_{2}$ have not yet significantly overlapped with metal orbitals, this must be the case. But at what distance from the surface does the spin-quenching take place and is it possible that a 

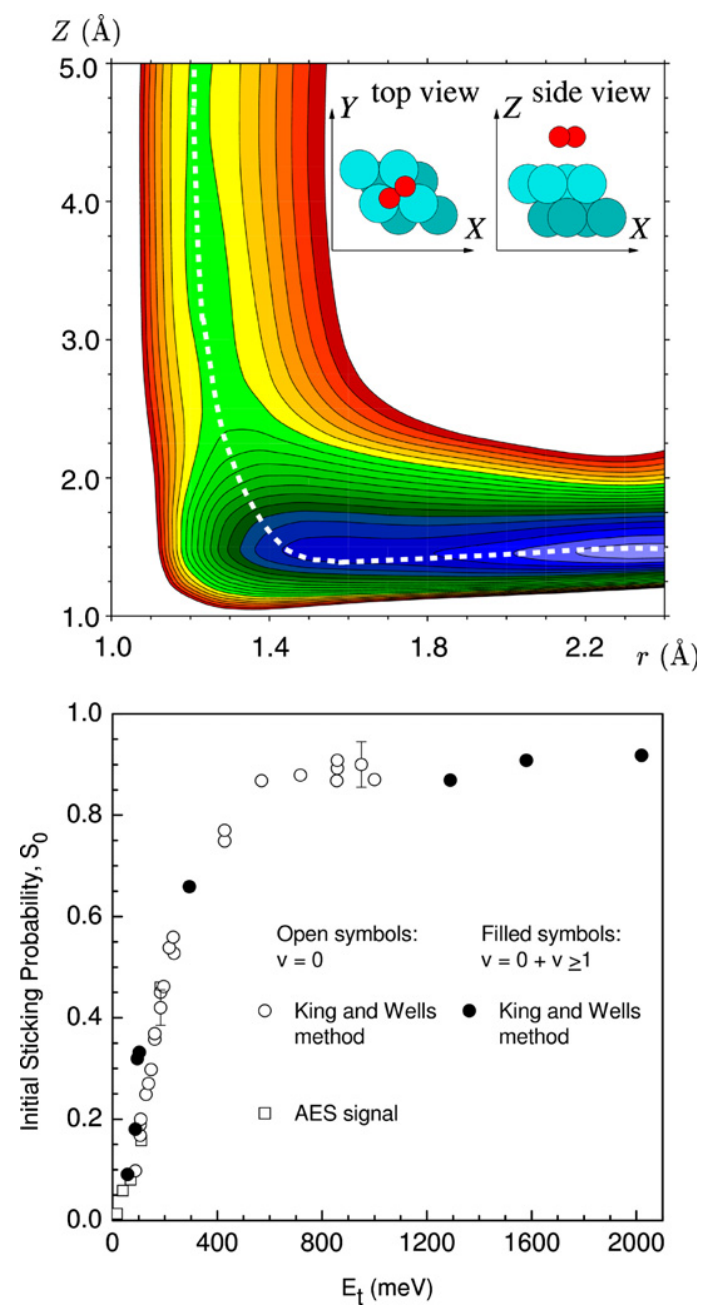

Fig. 23. Adiabatic DFT calculations of the $\mathrm{O}_{2}$ approach to $\mathrm{Al}(111)$ showing the minimum energy path to products. The reaction appears to proceed without a barrier. Experiment shows the reaction is strongly enhanced by $E_{I}$ of translation (motion along the $z$-axis of the upper panel). Adapted from Refs. [347,346].

triplet barrier can develop before this happens? Behler et al. have developed a spin-constrained DFT approach that allows calculation of a triplet and a singlet potential energy surface. These surfaces correlate to $\mathrm{O}_{2}\left(X^{3} \Sigma\right)$ and $\mathrm{O}_{2}\left(a^{1} \Delta\right)$ as $z$ goes to infinity. Furthermore, the triplet surface that is indeed the ground state at large $z$ exhibits a significant barrier to dissociation. Modeling the singlet-triplet seam crossing probabilities allows an accurate reproduction of the observed translational promotion of the reaction.

These results suggest that we have to constantly be on the lookout for errors in the potential energy surface due to the ways in which DFT handles (or fails to handle) spin-selection rules. This point is also supported by the work described above of $\mathrm{H}$ adsorption to $\mathrm{Cu}\left(\begin{array}{lll}1 & 1 & 1\end{array}\right)$ and the creation of chemi-currents. It is possible that similar electron excitation dynamics to those found in chemi-currents are at play for $\mathrm{O}_{2}$ dissociation on $\mathrm{Al}$ 
after it has passed the reaction barrier. Thus, while not influencing the reaction probability, the mobility of the product $\mathrm{O}$-atoms formed on the $\mathrm{Al}\left(\begin{array}{ll}1 & 1\end{array}\right)$ surface could perhaps be greatly reduced [348-350].

\section{Implications for our way forward}

We hope the reader will have gotten some appreciation for the exciting developments concerning our advancing knowledge of the nonadiabatic molecular interactions at solid surfaces. Theoretical methods (at least within the adiabatic limit) have become so sophisticated that comparison to surface chemistry experiments is highly valuable. This provides real opportunities to extract detailed and fundamental understanding from experimental work. Furthermore, the kinds and qualities of experimental tools now available are inspiring us to carry out ever more sophisticated and well-designed experiments. But the times are even more exciting. As we delve into a detailed look at the jiggling atoms involved in energy transfer at surfaces, we find that jiggling atoms lead to "jiggling electrons". The role of excited electronic states appears to be a special unanticipated characteristic of energy transfer and chemical reactions at surfaces that we have only just begun to understand. Experimental observations on many fronts have shown that our standard adiabatic approach using DFT has serious flaws for the problems we want to address. Clearly new theories will be needed in the future that go beyond the limitations of adiabatic DFT. Such theories are also essential for many other reasons. A first principles understanding of surface (or indeed all of solid state) photochemistry will require such theories. Developing this understanding will provide much better design and evaluation of the potential of important industrial processes like photocatalysis and photovoltaics.

More specifically, we see areas of research that offer immediate rewards. For example, while the emphasis of this review has been on the special electronic properties present in collisions at solid surfaces, especially metal surfaces, and the problems our present theories have to account for these, it is also worth noting that far too little has been done to advance our understanding of the role of phonons to the level of the chemistry of lattice distortion. This will be a rich area for future work as computational methods and infrastructure continue to improve. A good example of recent work is $\mathrm{H}$ adsorption to Graphite, where lattice distortions are an essential part of the carbon rehybridization necessary to form the $\mathrm{C}-\mathrm{H}$ bond [351-355]. Dissociative adsorption of $\mathrm{H}_{2}$ on $\mathrm{Si}$ is another such example [356]. Development of exact theories of anharmonic phonon coupling, which treat the multidimensional vibrational coupling problem is also needed. The beginnings of one approach in this direction appears promising [357,358].

The role of electronic excitations up to now has not been proven to affect the rate of surface chemical reactions. But this is (in our opinion) only a matter of time. Indeed, the long standing problem of $\mathrm{H}$-atom adsorption gives an indication of where the first conclusive work might come. It has long been understood that due to the large mass-mismatch between $\mathrm{H}$ and the atoms making up most solid surfaces, the sticking of $\mathrm{H}$ to those surfaces could not be easily explained by translational energy transfer to phonons. Yet it is an experimental fact that $\mathrm{H}$-atom sticking coefficients to many metals is close to unity. Indeed, Newns and Nørskov wrote papers more than 25 years ago essentially assuming that EHP excitation was the dominant energy transfer mechanism allowing H-atom sticking at metal surfaces [73,359]. Furthermore, Newns pointed out that the electronic excitation was mass independent. Yet, there remains no definitive experiment showing that 
sticking is influenced by electron hole pair excitation. A vigorous effort to find electronically nonadiabatic coupling of incidence translational energy to EHP excitation would be very desirable.

Another direction for the future will probe how modifications to the electronic structure of the solid influence rates of chemical reactions through electronically nonadiabatic couplings. An intriguing study on the thermal desorption of $\mathrm{Br}$ (and other halogens) from Halogen-terminated $\mathrm{Si}$, shows that the chemical reaction rates can depend on the level of doping in the $\mathrm{Si}[360,361]$. This has yet to be thoroughly understood, yet the implications are stunning - exploiting the power of semiconductor fabrication and solid state band structure manipulation to influence heterogeneous catalysis.

Energy transfer on complex surfaces represents another important horizon for future research. It is now well-known that surface defects can play an important role in chemical reaction rates at surfaces; perhaps the best know example being the enhanced rates of $\mathrm{N}_{2}$ [362-364] and $\mathrm{NO}[365,366]$ dissociation at $\mathrm{Ru}$ steps and $\mathrm{O}_{2}$ dissociation on $\mathrm{Pt}$ steps [367]. Indeed, heterogeneous catalysis is typically carried out on complex interfacial structures that may bear little resemblance to a single crystal metal surface. Furthermore, the enhanced catalytic activity of noble metals when reduced to nanometer-sized particles [368-371] is yet to be well understood, further emphasizing the importance of complex surfaces. Up to now there are few examples of work that probes the how energy-transfer depends on these subtle aspects of surface morphology. Future studies in this direction certainly promise new insights and discoveries.

In conclusion, we hope to have provided the reader with an overview of present day methodology and a collage of interesting examples of work representing the state-ofthe-art in the title field. We hope to have conveyed the underlying impression that the study of molecular interactions at solid surfaces is presently at a remarkably interesting point as the cooperation between experimentalists and theorists has never before been so productive. This has allowed and will allow our basic understanding to advance at a rapid pace. Furthermore, there are still many areas of research that have not been seriously studied where new approaches are needed providing many opportunities for scientists of different stripes to make important contributions to advance the field.

\section{Acknowledgements}

Some of the work described in this paper was supported by the Partnership for International Research and Education - for Electronic Chemistry and Catalysis at Interfaces NSF Grant Number OISE-0530268, The National Science Foundation under Grant Number CHE-0454806, the Department of Energy Office of Basic Energy Sciences, under Grant Number DE-FG02-03ER15441, and the Air Force Office of Scientific Research, under Grant Number FA9550-07-1-0206.

\section{References}

[1] See for example Phil. Mag. 4 (1852), from Sir William Thomson, Mathematcial and Physical Papers, vol. 1, pp. $174 \mathrm{ff}$.

[2] Indeed, Thomson coined the term 'kinetic energy', 1856.

[3] Bernoulli Daniel, Hydrodynamica, sive de viribus et motibus fluidorum commentarii, Commentaries Concerning the Pressure and Motion of Fluids, Johann Reinhold Dulsseker, Strasbourg, 1738. 
[4] T. Kurten, M. Biczysko, T. Rajamaki, K. Laasonen, L. Halonen, Computational study of the adsorption energetics and vibrational wavenumbers of $\mathrm{NH}_{3}$ adsorbed on the $\mathrm{Ni}(111)$ surface, J. Phys. Chem. B 109 (2005) 8954-8960.

[5] G. Onida, L. Reining, A. Rubio, Electronic excitations: density-functional versus many-body Green'sfunction approaches, Rev. Mod. Phys. 74 (2002) 601-659.

[6] W.D. Schone, Theoretical determination of electronic lifetimes in metals, Prog. Surf. Sci. 82 (2007) $161-192$.

[7] D. Sanchez-Portal, Slab calculations and Green's function recursive methods combined to study the electronic structure of surfaces: application to $\mathrm{Cu}(111)-(4 \times 4)-\mathrm{Na}$, Prog. Surf. Sci. 82 (2007) $313-335$.

[8] J. Behler, B. Delley, K. Reuter, M. Scheffler, Nonadiabatic potential-energy surfaces by constrained density-functional theory, Phys. Rev. B 75 (2007).

[9] P. Mori-Sanchez, A.J. Cohen, W.T. Yang, Self-interaction-free exchange-correlation functional for thermochemistry and kinetics, J. Chem. Phys. 124 (2006).

[10] Q. Wu, T. Van Voorhis, Extracting electron transfer coupling elements from constrained density functional theory, J. Chem. Phys. 125 (2006).

[11] E. Livshits, R. Baer, A well-tempered density functional theory of electrons in molecules, Phys. Chem. Chem. Phys. 9 (2007) 2932-2941.

[12] E. Hasselbrink, How non-adiabatic are surface dynamical processes? Curr. Opin. Solid State Mater. Sci. 10 (2006) 192-204.

[13] K.W. Kolasinski, Non-adiabatic and ultrafast dynamics of hydrogen adsorbed on silicon, Curr. Opin. Solid State Mater. Sci. 8 (2004) 353-366.

[14] B. Gumhalter, Single- and multiphonon atom-surface scattering in the quantum regime, Phys. Rep. 351 (2001) $1-159$.

[15] J.C. Tully, Chemical dynamics at metal surfaces, Annu. Rev. Phys. Chem. 51 (2000) 153-178.

[16] C.R. Arumainayagam, R.J. Madix, Molecular-beam studies of gas-surface collision dynamics, Prog. Surf. Sci. 38 (1991) 1-102.

[17] D.R. Yarkony, Current issues in nonadiabatic chemistry, J. Phys. Chem. 100 (1996) 18612-18628.

[18] C.D. Lindstrom, X.Y. Zhu, Photoinduced electron transfer at molecule-metal interfaces, Chem. Rev. 106 (2006) 4281-4300.

[19] K. Watanabe, D. Menzel, N. Nilius, H.J. Freund, Photochemistry on metal nanoparticles, Chem. Rev. 106 (2006) 4301-4320.

[20] G. Ertl, Dynamics of reactions at surfaces, Adv. Catal. 45 (2000) 1-69.

[21] I. Harrison, Photochemical exploration of reaction dynamics at catalytic metal surfaces: from ballistics to statistics, Acc. Chem. Res. 31 (1998) 631-639.

[22] R.B. Gerber, A. Amirav, Dynamics of dissociation and energy-transfer in molecular-collisions with solidsurfaces, J. Phys. Chem. 90 (1986) 4483-4491.

[23] S.T. Ceyer, Dissociative chemisorption - dynamics and mechanisms, Annu. Rev. Phys. Chem. 39 (1988) 479-510.

[24] U. Heinzmann, S. Holloway, A.W. Kleyn, R.E. Palmer, K.J. Snowdon, Orientation in molecule--surface interactions, J. Phys. - Condens. Matter 8 (1996) 3245-3269.

[25] H. Petek, S. Ogawa, Surface femtochemistry: observation and quantum control of frustrated desorption of alkali atoms from noble metals, Annu. Rev. Phys. Chem. 53 (2002) 507-531.

[26] R.E. Palmer, Electron-molecule dynamics at surfaces, Prog. Surf. Sci. 41 (1992) 51-108.

[27] X.L. Zhou, X.Y. Zhu, J.M. White, Photochemistry at adsorbate metal interfaces, Surf. Sci. Rep. 13 (1991) $73-220$.

[28] Y.J. Chabal, Surface infrared-spectroscopy, Surf. Sci. Rep. 8 (1988) 211-357.

[29] P. Jakob, Y.J. Chabal, Chemical etching of vicinal Si(1 11$)$ - dependence of the surface-structure and the hydrogen termination on the $\mathrm{pH}$ of the etching solutions, J. Chem. Phys. 95 (1991) 2897-2909.

[30] R. Ryberg, Carbon-monoxide adsorbed on $\mathrm{Cu}(100)$ studied by infrared-spectroscopy, Surf. Sci. 114 (1982) $627-641$.

[31] H. Ueba, Vibrational lineshapes of adsorbates on solid-surfaces, Prog. Surf. Sci. 22 (1986) 181-321.

[32] J.W. Gadzuk, A.C. Luntz, On vibrational lineshapes of adsorbed molecules, Surf. Sci. 144 (1984) $429-450$.

[33] P. Avouris, B.N.J. Persson, Excited-states at metal-surfaces and their nonradiative relaxation, J. Phys. Chem. 88 (1984) 837-848.

[34] R. Ryberg, Infrared-spectroscopy of molecules adsorbed on metal-surfaces, Adv. Chem. Phys. 76 (1989) 1-44.

[35] P. Hollins, J. Pritchard, Infrared studies of chemisorbed layers on single-crystals, Prog. Surf. Sci. 19 (1985) $275-350$. 
[36] M.A. Kozkusner, Surf. Sci. 81 (1979).

[37] B.N.J. Persson, M. Persson, Vibrational lifetime for $\mathrm{CO}$ adsorbed on $\mathrm{Cu}(100)$, Solid State Commun. 36 (1980) $175-179$.

[38] B.N.J. Persson, R. Ryberg, Vibrational phase relaxation at surfaces - CO on Ni(111), Phys. Rev. Lett. 54 (1985) 2119-2122.

[39] R. Ryberg, Vibrational-energy relaxation of the metal-molecule stretch mode - CO on $\operatorname{Pt}(111)$, Phys. Rev. B 40 (1989) 5849-5851.

[40] B.N.J. Persson, R. Ryberg, Brownian-motion and vibrational phase relaxation at surfaces - CO on Ni(1 11 ), Phys. Rev. B 32 (1985) 3586-3596.

[41] R. Ryberg, Vibrational line-shape of chemisorbed CO, Phys. Rev. B 32 (1985) 2671-2673.

[42] W. Erley, B.N.J. Persson, Vibrational lineshapes for NO on Ni(111), Surf. Sci. 218 (1989) 494-506.

[43] E.J. Heilweil, M.P. Casassa, R.R. Cavanagh, J.C. Stephenson, Vibrational deactivation of surface OH chemisorbed on $\mathrm{SiO}_{2}$ - solvent effects, J. Chem. Phys. 82 (1985) 5216-5231.

[44] E.J. Heilweil, M.P. Casassa, R.R. Cavanagh, J.C. Stephenson, Time-resolved vibrational-energy relaxation of surface adsorbates, J. Vacuum Sci. Technol. B 3 (1985) 1471-1473.

[45] M.P. Casassa, E.J. Heilweil, J.C. Stephenson, R.R. Cavanagh, Time-resolved measurements of vibrationalrelaxation at surfaces, J. Electron. Spectrosc. Relat. Phenom. 38 (1986) 257-265.

[46] M.P. Casassa, E.J. Heilweil, J.C. Stephenson, R.R. Cavanagh, Time-resolved measurements of OH $(v=1)$ vibrational-relaxation on $\mathrm{SiO}_{2}$ surfaces - isotope and temperature-dependence, J. Chem. Phys. 84 (1986) 2361-2364.

[47] R.R. Cavanagh, M.P. Casassa, E.J. Heilweil, J.C. Stephenson, Vibrational-relaxation of adsorbed molecules - comparison with relaxation rates of model compounds, J. Vacuum Sci. Technol. A - Vacuum Surf. Films 5 (1987) 469-472.

[48] G.E. Ewing, Energy-flow from excited molecules on salt surfaces, Acc. Chem. Res. 25 (1992) 292-299.

[49] G.E. Ewing, A model system for the study of structure and dynamics of molecules on surfaces - CO on $\mathrm{NaCl}(100)$, Int. Rev. Phys. Chem. 10 (1991) 391-425.

[50] H.C. Chang, G.E. Ewing, Infrared fluorescence from a monolayer of $\mathrm{CO}$ on $\mathrm{NaCl}(100)$, Phys. Rev. Lett. 65 (1990) 2125-2128.

[51] H. Ueba, Vibrational relaxation and pump-probe spectroscopies of adsorbates on solid surfaces, Prog. Surf. Sci. 55 (1997) 115-179.

[52] M.B. Raschke, Y.R. Shen, Nonlinear optical spectroscopy of solid interfaces, Curr. Opin. Solid State Mater. Sci. 8 (2004) 343-352.

[53] J. Kubota, K. Domen, Study of the dynamics of surface molecules by time-resolved sum-frequency generation spectroscopy, Anal. Bioanal. Chem. 388 (2007) 17-27.

[54] P. Dumas, M.K. Weldon, Y.J. Chabal, G.P. Williams, Molecules at surfaces and interfaces studied using vibrational spectroscopies and related techniques, Surf. Rev. Lett. 6 (1999) 225-255.

[55] R.R. Cavanagh, E.J. Heilweil, J.C. Stephenson, Time-domain measurements of vibrational-relaxation at surfaces - CO $(v=1)$ in metal-carbonyl systems, J. Electron. Spectrosc. Relat. Phenom. 45 (1987) 31-40.

[56] E.J. Heilweil, J.C. Stephenson, R.R. Cavanagh, Measurements of CO $(v=1)$ population lifetimes - metalcarbonyl cluster compounds supported on $\mathrm{SiO}_{2}$, J. Phys. Chem. 92 (1988) 6099-6103.

[57] E.J. Heilweil, M.P. Casassa, R.R. Cavanagh, J.C. Stephenson, Picosecond vibrational-energy transfer studies of surface adsorbates, Annu. Rev. Phys. Chem. 40 (1989) 143-171.

[58] J.D. Beckerle, M.P. Casassa, R.R. Cavanagh, E.J. Heilweil, J.C. Stephenson, Ultrafast infrared response of adsorbates on metal-surfaces - vibrational lifetime of $\mathrm{CO} / \mathrm{Pt}(111)$, Phys. Rev. Lett. 64 (1990) 2090-2093.

[59] R.R. Cavanagh, D.S. King, J.C. Stephenson, T.F. Heinz, Dynamics of nonthermal reactions - femtosecond surface-chemistry, J. Phys. Chem. 97 (1993) 786-798.

[60] R.R. Cavanagh, E.J. Heilweil, J.C. Stephenson, Time-resolved measurements of energy-transfer at surfaces, Surf. Sci. 300 (1994) 643-655.

[61] T.A. Germer, J.C. Stephenson, E.J. Heilweil, R.R. Cavanagh, Picosecond time-resolved adsorbate response to substrate heating - spectroscopy and dynamics of $\mathrm{CO} / \mathrm{Cu}(100)$, J. Chem. Phys. 101 (1994) 1704-1716.

[62] K. Kuhnke, M. Morin, P. Jakob, N.J. Levinos, Y.J. Chabal, A.L. Harris, Vibrational-energy transfer among adsorbate modes - picosecond dynamics on stepped H/Si(111), J. Chem. Phys. 99 (1993) 6114 6125.

[63] A.L. Harris, K. Kuhnke, M. Morin, P. Jakob, N.J. Levinos, Y.J. Chabal, Vibrational-energy flow at stepped H/Si(1 11 ) - phonons, dipoles and screening, Faraday Discuss. (1993) 217-226. 
[64] M. Morin, K. Kuhnke, P. Jakob, Y.J. Chabal, N.J. Levinos, A.L. Harris, Interadsorbate vibrational-energy flow on stepped vicinal H/Si(111)) surfaces, J. Electron. Spectrosc. Relat. Phenom. 64-65 (1993) 11-21.

[65] M. Morin, P. Jakob, N.J. Levinos, Y.J. Chabal, A.L. Harris, Vibrational-energy transfer on hydrogenterminated vicinal Si(111) surfaces - interadsorbate energy-flow, J. Chem. Phys. 96 (1992) 6203-6212.

[66] M. Morin, N.J. Levinos, A.L. Harris, Vibrational-energy transfer of $\mathrm{CO} / \mathrm{Cu}(100)$ - nonadiabatic vibration electron coupling, J. Chem. Phys. 96 (1992) 3950-3956.

[67] Z.H. Liu, L.C. Feldman, N.H. Tolk, Z.Y. Zhang, P.I. Cohen, Desorption of H from Si(111) by resonant excitation of the Si-H vibrational stretch mode, Science 312 (2006) 1024-1026.

[68] G.K. Paramonov, I. Andrianov, P. Saalfrank, Breaking relaxing bonds at a H:Si(100)-(2×1) surface with infrared laser pulses, J. Phys. Chem. C 111 (2007) 5432-5440.

[69] I. Andrianov, P. Saalfrank, Free vibrational relaxation of H adsorbed on a Si(100) surface investigated with the multi-configurational time-dependent Hartree method, Chem. Phys. Lett. 433 (2006) 91-96.

[70] R. Brako, D.M. Newns, Theory of electronic processes in atom scattering from surfaces, Rep. Prog. Phys. 52 (1989) 655-697.

[71] B. Hammer, Special sites at noble and late transition metal catalysts, Top. Catal. 37 (2006) 3-16.

[72] A. Yoshimori, K. Makoshi, Time-dependent Newns-Anderson model, Prog. Surf. Sci. 21 (1986) 251294.

[73] R. Brako, D.M. Newns, Electron-hole mechanism for sticking of adsorbates - soluble model, Solid State Commun. 33 (1980) 713-715.

[74] Y.G. Li, G. Wahnstrom, Nonadiabatic effects in hydrogen diffusion in metals, Phys. Rev. Lett. 68 (1992) 3444-3447.

[75] H. Kasai, A. Okiji, Vibrational-excitation dynamics in molecule surface scattering, Surf. Sci. 242 (1991) 394-399.

[76] H. Kasai, A. Okiji, Electron-hole pair mechanism for excitation of intramolecular vibrations in molecule surface scattering, Surf. Sci. 225 (1990) L33-L38.

[77] G. Wahnstrom, Role of phonons and electron-hole pairs in hydrogen diffusion on a corrugated metalsurface, Chem. Phys. Lett. 163 (1989) 401-406.

[78] K.L. Sebastian, Electrochemical electron-transfer - accounting for electron-hole excitations in the metalelectrode, J. Chem. Phys. 90 (1989) 5056-5067.

[79] J.C. Tully, Stochastic trajectory simulations of vibrational-energy flow at surfaces, J. Electron. Spectrosc. Relat. Phenom. 45 (1987) 381-389.

[80] K. Schonhammer, O. Gunnarsson, Electronic friction and covalent chemisorption, Phys. Rev. B 27 (1983) 5113-5115.

[81] P. Minnhagen, Excitation probability in a time-dependent external potential, J. Phys. C - Solid State Phys. 15 (1982) 2293-2303.

[82] K. Schonhammer, O. Gunnarsson, Sticking probability on metal-surfaces - contribution from electronhole-pair excitations, Phys. Rev. B 22 (1980) 1629-1637.

[83] A. Nourtier, Friction coefficient of atoms near a metal-surface, J. Phys. 38 (1977) 479-502.

[84] W.L. Schaich, Model calculation of Brownian-motion parameters at a metal-surface, Surf. Sci. 49 (1975) 221-235.

[85] E.G. Dagliano, P. Kumar, W. Schaich, H. Suhl, Brownian-motion model of interactions between chemical species and metallic electrons - bootstrap derivation and parameter evaluation, Phys. Rev. B 11 (1975) 2122-2143.

[86] W.L. Schaich, Brownian-motion model of surface chemical-reactions - derivation in large mass limit, J. Chem. Phys. 60 (1974) 1087-1093.

[87] M. Headgordon, J.C. Tully, Molecular-dynamics with electronic frictions, J. Chem. Phys. 103 (1995) $10137-10145$.

[88] J.R. Trail, M.C. Graham, D.M. Bird, Electronic damping of molecular motion at metal surfaces, Comput. Phys. Commun. 137 (2001) 163-173.

[89] J.C. Tully, M. Gomez, M. Headgordon, Electronic and phonon mechanisms of vibrational-relaxation - CO on $\mathrm{Cu}(100)$, J. Vacuum Sci. Technol. A - Vacuum Surf. Films 11 (1993) 1914-1920.

[90] V. Krishna, J.C. Tully, Vibrational lifetimes of molecular adsorbates on metal surfaces, J. Chem. Phys. 125 (2006).

[91] A.C. Luntz, M. Persson, S. Wagner, C. Frischkorn, M. Wolf, Femtosecond laser induced associative desorption of $\mathrm{H}_{2}$ from $\mathrm{Ru}(0001)$ : comparison of "first principles" theory with experiment, J. Chem. Phys. 124 (2006). 
[92] A.C. Luntz, M. Persson, G.O. Sitz, Theoretical evidence for nonadiabatic vibrational deexcitation in $\mathrm{H}_{2}\left(\mathrm{D}_{2}\right)$ state-to-state scattering from $\mathrm{Cu}(100)$, J. Chem. Phys. 124 (2006).

[93] L. Diekhoner, L. Hornekaer, H. Mortensen, E. Jensen, A. Baurichter, V.V. Petrunin, A.C. Luntz, Indirect evidence for strong nonadiabatic coupling in $\mathrm{N}_{2}$ associative desorption from and dissociative adsorption on Ru(0001), J. Chem. Phys. 117 (2002) 5018-5030.

[94] J.T. Kindt, J.C. Tully, M. Head-Gordon, M.A. Gomez, Electron-hole pair contributions to scattering, sticking, and surface diffusion: CO on Cu(100), J. Chem. Phys. 109 (1998) 3629-3636.

[95] B.B. Smith, J.T. Hynes, Electronic friction and electron-transfer rates at metallic electrodes, J. Chem. Phys. 99 (1993) 6517-6530.

[96] J. Gudde, U. Hofer, Dynamics of femtosecond-laser-induced lateral motion of an adsorbate: O on vicinal Pt(1 1 1), J. Phys. - Condens. Matter 18 (2006) S1409-S1424.

[97] C. Springer, M. Headgordon, J.C. Tully, Simulations of femtosecond laser-induced desorption of CO from $\mathrm{Cu}(100)$, Surf. Sci. 320 (1994) L57-L62.

[98] P. Saalfrank, Quantum dynamical approach to ultrafast molecular desorption from surfaces, Chem. Rev. 106 (2006) 4116-4159.

[99] C. Frischkorn, M. Wolf, Femtochemistry at metal surfaces: nonadiabatic reaction dynamics, Chem. Rev. 106 (2006) 4207-4233.

[100] N. Shenvi, S. Roy, P. Parandekar, J. Tully, Vibrational relaxation of NO on Au(1 11 1) via electron-hole pair generation, J. Chem. Phys. 125 (2006).

[101] P.W. Anderson, Localized magnetic states in metals, Phys. Rev. 1 (1961) 41-53.

[102] D.M. Newns, Self-consistent model of hydrogen chemisorption, Phys. Rev. 178 (1969) 1123-1135.

[103] D.M. Newns, Electron-hole pair mechanism for excitation of intramolecular vibrations in molecule surface scattering, Surf. Sci. 171 (1986) 600-614.

[104] D.C. Langreth, P. Nordlander, Derivation of a master equation for charge-transfer processes in atomsurface collisions, Phys. Rev. B 43 (1991) 2541-2557.

[105] W. Bloss, D. Hone, Theory of charge-exchange scattering from surfaces, Surf. Sci. 72 (1978) $277-297$.

[106] R. Brako, D.M. Newns, Charge-exchange in atom-surface scattering - thermal versus quantum-mechanical non-adiabaticity, Surf. Sci. 108 (1981) 253-270.

[107] M.S. Mizielinski, D.M. Bird, M. Persson, S. Holloway, Electronic nonadiabatic effects in the adsorption of hydrogen atoms on metals, J. Chem. Phys. 122 (2005) 084710.

[108] M. Forsblom, M. Persson, Vibrational lifetimes of cyanide and carbon monoxide on noble and transition metal surfaces, J. Chem. Phys. 127 (2007).

[109] M.S. Mizielinski, D.M. Bird, M. Persson, S. Holloway, Spectrum of electronic excitations due to the adsorption of atoms on metal surfaces, J. Chem. Phys. 126 (2007).

[110] J.R. Trail, D.M. Bird, M. Persson, S. Holloway, Electron-hole pair creation by atoms incident on a metal surface, J. Chem. Phys. 119 (2003) 4539-4549.

[111] J.R. Trail, M.C. Graham, D.M. Bird, M. Persson, S. Holloway, Energy loss of atoms at metal surfaces due to electron-hole pair excitations: first-principles theory of "chemicurrents", Phys. Rev. Lett. 88 (2002).

[112] D.N. Denzler, C. Frischkorn, C. Hess, M. Wolf, G. Ertl, Electronic excitation and dynamic promotion of a surface reaction, Phys. Rev. Lett. 91 (2003).

[113] S. Wagner, C. Frischkorn, M. Wolf, M. Rutkowski, H. Zacharias, A.C. Luntz, Energy partitioning in the femtosecond-laser-induced associative $\mathrm{D}_{2}$ desorption from $\mathrm{Ru}(0001)$, Phys. Rev. B 72 (2005).

[114] S. Funk, M. Bonn, D.N. Denzler, C. Hess, M. Wolf, G. Ertl, Desorption of CO from Ru(001) induced by near-infrared femtosecond laser pulses, J. Chem. Phys. 112 (2000) 9888-9897.

[115] C. Hess, M. Wolf, S. Roke, M. Bonn, Femtosecond time-resolved vibrational SFG spectroscopy of CO/ Ru(001), Surf. Sci. 502 (2002) 304-312.

[116] C. Hess, M. Bonn, S. Funk, M. Wolf, Hot-band excitation of CO chemisorbed on Ru(001) studied with broadband-IR sum-frequency generation, Chem. Phys. Lett. 325 (2000) 139-145.

[117] M. Bonn, M. Wolf, Optimizing vibrational population transfer at surfaces through infrared excitation, Bull. Chem. Soc. Jpn. 75 (2002) 1005-1010.

[118] M. Bonn, C. Hess, M. Wolf, The dynamics of vibrational excitations on surfaces: CO on Ru(001), J. Chem. Phys. 115 (2001) 7725-7735.

[119] M. Bonn, S. Funk, C. Hess, D.N. Denzler, C. Stampfl, M. Scheffler, M. Wolf, G. Ertl, Phonon- versus electron-mediated desorption and oxidation of CO on Ru(0001), Science 285 (1999) 1042-1045.

[120] K. AlShamery, H.J. Freund, Laser-stimulated desorption from surfaces, Curr. Opin. Solid State Mater. Sci. 1 (1996) 622-629. 
[121] C. Hess, S. Funk, M. Bonn, D.N. Denzler, M. Wolf, G. Ertl, Femtosecond dynamics of chemical reactions at surfaces, Appl. Phys. A - Mater. Sci. Process. 71 (2000) 477-483.

[122] C. Hess, M. Wolf, M. Bonn, Direct observation of vibrational energy delocalization on surfaces: CO on Ru(001), Phys. Rev. Lett. 85 (2000) 4341-4344.

[123] M. Bonn, C. Hess, S. Funk, J.H. Miners, B.N.J. Persson, M. Wolf, G. Ertl, Femtosecond surface vibrational spectroscopy of $\mathrm{CO}$ adsorbed on $\mathrm{Ru}(001)$ during desorption, Phys. Rev. Lett. 84 (2000) 46534656.

[124] M. Bonn, D.N. Denzler, S. Funk, M. Wolf, S.S. Wellershoff, J. Hohlfeld, Ultrafast electron dynamics at metal surfaces: competition between electron-phonon coupling and hot-electron transport, Phys. Rev. B 61 (2000) 1101-1105.

[125] D.N. Denzler, C. Frischkorn, M. Wolf, G. Ertl, Surface femtochemistry: associative desorption of hydrogen from $\mathrm{Ru}(001)$ induced by electronic excitations, J. Phys. Chem. B 108 (2004) 14503-14510.

[126] C. Frischkorn, Microscopic understanding of an ultrafast photochemical surface reaction: $\mathrm{H}_{\mathrm{ads}}+\mathrm{H}_{\mathrm{ads}} \rightarrow \mathrm{H}_{2, \text { gas }}$ on $\mathrm{Ru}(001)$, Surf. Sci. 593 (2005) 67-78.

[127] M. Lawrenz, P. Kratzer, C.H. Schwalb, M. Durr, U. Hofer, Diffusion pathways of hydrogen across the steps of a vicinal $\mathrm{Si}(001)$ surface, Phys. Rev. B 75 (2007).

[128] C.H. Schwalb, M. Lawrenz, M. Durr, U. Hofer, Real-space investigation of fast diffusion of hydrogen on $\mathrm{Si}(001)$ by a combination of nanosecond laser heating and STM, Phys. Rev. B 75 (2007).

[129] K. Stepan, M. Durr, J. Gudde, U. Hofer, Laser-induced diffusion of oxygen on a stepped Pt(1 111$)$ surface, Surf. Sci. 593 (2005) 54-66.

[130] K. Stepan, J. Gudde, U. Hofer, Time-resolved measurement of surface diffusion induced by femtosecond laser pulses, Phys. Rev. Lett. 94 (2005).

[131] H. Hou, S.J. Gulding, C.T. Rettner, A.M. Wodtke, D.J. Auerbach, The stereodynamics of a gas-surface reaction, Science 277 (1997) 80-82.

[132] H.A. Michelsen, D.J. Auerbach, A critical-examination of data on the dissociative adsorption and associative desorption of hydrogen at copper surfaces, J. Chem. Phys. 94 (1991) 7502-7520.

[133] C.T. Rettner, H.A. Michelsen, D.J. Auerbach, From quantum-state-specific dynamics to reaction-rates the dominant role of translational energy in promoting the dissociation of $\mathrm{D}_{2}$ on $\mathrm{Cu}(111)$ under equilibrium conditions, Faraday Discuss. (1993) 17-31.

[134] C.T. Rettner, H.A. Michelsen, D.J. Auerbach, Vibrational effects in the dissociation and scattering of hydrogen at a $\mathrm{Cu}(1111)$ surface, J. Electron. Spectrosc. Relat. Phenom. 64-5 (1993) 543-554.

[135] C.T. Rettner, H.A. Michelsen, D.J. Auerbach, Dynamics of the desorption of $\mathrm{D}_{2}$ and $\mathrm{H}_{2}$ from Cu(111), J. Vacuum Sci. Technol. A - Vacuum Surf. Films 11 (1993) 1901-1906.

[136] H.A. Michelsen, C.T. Rettner, D.J. Auerbach, R.N. Zare, Effect of rotation on the translational and vibrational-energy dependence of the dissociative adsorption of $\mathrm{D}_{2}$ on $\mathrm{Cu}(111)$, J. Chem. Phys. 98 (1993) 8294-8307.

[137] D.J. Auerbach, C.T. Rettner, H.A. Michelsen, Interaction dynamics of hydrogen at a Cu(111) surface, Surf. Sci. 283 (1993) 1-8.

[138] H.A. Michelsen, C.T. Rettner, D.J. Auerbach, State-specific dynamics of $\mathrm{D}_{2}$ desorption from Cu(111) - the role of molecular rotational motion in activated adsorption-desorption dynamics, Phys. Rev. Lett. 69 (1992) 2678-2681.

[139] C.T. Rettner, D.J. Auerbach, H.A. Michelsen, Dynamic studies of the interaction of $\mathrm{D}_{2}$ with a Cu(111) surface, J. Vacuum Sci. Technol. A - Vacuum Surf. Films 10 (1992) 2282-2286.

[140] H.A. Michelsen, C.T. Rettner, D.J. Auerbach, On the influence of surface-temperature on adsorption and desorption in the $\mathrm{D}_{2} / \mathrm{Cu}(111)$ system, Surf. Sci. 272 (1992) 65-72.

[141] C.T. Rettner, D.J. Auerbach, H.A. Michelsen, Observation of direct vibrational-excitation in collisions of $\mathrm{H}_{2}$ and $\mathrm{D}_{2}$ with a $\mathrm{Cu}(111)$ surface, Phys. Rev. Lett. 68 (1992) 2547-2550.

[142] C.T. Rettner, D.J. Auerbach, H.A. Michelsen, Role of vibrational and translational energy in the activated dissociative adsorption of $\mathrm{D}_{2}$ on $\mathrm{Cu}(111)$, Phys. Rev. Lett. 68 (1992) 1164-1167.

[143] C.T. Rettner, H.A. Michelsen, D.J. Auerbach, C.B. Mullins, Dynamics of recombinative desorption angular-distributions of $\mathrm{H}_{2}, \mathrm{HD}$, and $\mathrm{D}_{2}$ desorbing from $\mathrm{Cu}(111)$, J. Chem. Phys. 94 (1991) 7499-7501.

[144] F. Budde, T.F. Heinz, A. Kalamarides, M.M.T. Loy, J.A. Misewich, Vibrational distributions in desorption induced by femtosecond laser-pulses - coupling of adsorbate vibration to substrate electronic excitation, Surf. Sci. 283 (1993) 143-157.

[145] J.W. Gadzuk, Hot-electron femtochemistry at surfaces: on the role of multiple electron processes in desorption, Chem. Phys. 251 (2000) 87-97. 
[146] J.W. Gadzuk, Intramolecular dynamics due to electron transitions: from photoelectron spectroscopy to femtochemistry, J. Electron. Spectrosc. Relat. Phenom. 99 (1999) 321-333.

[147] J.W. Gadzuk, Resonance-assisted hot electron femtochemistry at surfaces, Phys. Rev. Lett. 76 (1996) 4234 4237.

[148] J.A. Stroscio, F. Tavazza, J.N. Crain, R.J. Celotta, A.M. Chaka, Electronically induced atom motion engineered $\mathrm{CoCu}$ nanostructures, Science 313 (2006) 948-951.

[149] J.A. Stroscio, D.M. Eigler, Atomic and molecular manipulation with the scanning tunneling microscope, Science 254 (1991) 1319-1326.

[150] B.C. Stipe, M.A. Rezaei, W. Ho, Coupling of vibrational excitation to the rotational motion of a single adsorbed molecule, Phys. Rev. Lett. 81 (1998) 1263-1266.

[151] B.C. Stipe, M.A. Rezaei, W. Ho, Single-molecule vibrational spectroscopy and microscopy, Science 280 (1998) 1732-1735.

[152] B.C. Stipe, M.A. Rezaei, W. Ho, Inducing and viewing the rotational motion of a single molecule, Science 279 (1998) 1907-1909.

[153] J. Gaudioso, H.J. Lee, W. Ho, Vibrational analysis of single molecule chemistry: ethylene dehydrogenation on Ni(1 10), J. Am. Chem. Soc. 121 (1999) 8479-8485.

[154] L.J. Lauhon, W. Ho, Single-molecule vibrational spectroscopy and microscopy: $\mathrm{CO}$ on $\mathrm{Cu}(001)$ and $\mathrm{Cu}(110)$, Phys. Rev. B 60 (1999) R8525-R8528.

[155] L.J. Lauhon, W. Ho, Single molecule thermal rotation and diffusion: acetylene on $\mathrm{Cu}(001)$, J. Chem. Phys. 111 (1999) 5633-5636.

[156] J. Gaudioso, L.J. Lauhon, W. Ho, Vibrationally mediated negative differential resistance in a single molecule, Phys. Rev. Lett. 85 (2000) 1918-1921.

[157] J.R. Hahn, H.J. Lee, W. Ho, Electronic resonance and symmetry in single-molecule inelastic electron tunneling, Phys. Rev. Lett. 85 (2000) 1914-1917.

[158] L.J. Lauhon, W. Ho, Electronic and vibrational excitation of single molecules with a scanning tunneling microscope, Surf. Sci. 451 (2000) 219-225.

[159] L.J. Lauhon, W. Ho, Single-molecule chemistry and vibrational spectroscopy: pyridine and benzene on $\mathrm{Cu}(001)$, J. Phys. Chem. A 104 (2000) 2463-2467.

[160] J. Gaudioso, W. Ho, Single-molecule vibrations, conformational changes, and electronic conductivity of five-membered heterocycles, J. Am. Chem. Soc. 123 (2001) 10095-10098.

[161] W. Ho, Single-molecule chemistry, J. Chem. Phys. 117 (2002) 11033-11061.

[162] X.H. Qiu, G.V. Nazin, W. Ho, Vibronic states in single molecule electron transport, Phys. Rev. Lett. 92 (2004).

[163] S.W. Wu, G.V. Nazin, X. Chen, X.H. Qiu, W. Ho, Control of relative tunneling rates in single molecule bipolar electron transport, Phys. Rev. Lett. 93 (2004).

[164] H.J. Lee, J.H. Lee, W. Ho, Vibronic transitions in single metalloporphyrins, Chemphyschem 6 (2005) $971-$ 975.

[165] G.V. Nazin, X.H. Qiu, W. Ho, Vibrational spectroscopy of individual doping centers in a monolayer organic crystal, J. Chem. Phys. 122 (2005).

[166] N.A. Pradhan, N. Liu, W. Ho, Vibronic spectroscopy of single $\mathrm{C}_{60}$ molecules and monolayers with the STM, J. Phys. Chem. B 109 (2005) 8513-8518.

[167] B.C. Stipe, M.A. Rezaei, W. Ho, A variable-temperature scanning tunneling microscope capable of singlemolecule vibrational spectroscopy, Rev. Sci. Instrum. 70 (1999) 137-143.

[168] N. Lorente, R. Rurali, H. Tang, Single-molecule manipulation and chemistry with the STM, J. Phys. Condens. Matter 17 (2005) S1049-S1074.

[169] T. Seideman, Current-driven dynamics in molecular-scale devices, J. Phys. - Condens. Matter 15 (2003) R521-R549.

[170] N. Lorente, H. Ueba, CO dynamics induced by tunneling electrons: differences on $\mathrm{Cu}(110)$ and $\mathrm{Ag}(110)$, Eur. Phys. J. D 35 (2005) 341-348.

[171] M. Persson, Theory of elastic and inelastic tunnelling microscopy and spectroscopy: $\mathrm{CO}$ on $\mathrm{Cu}$ revisited, Philos. Trans. Royal Soc. London Seri. A - Math. Phys. Eng. Sci. 362 (2004) 11731183.

[172] N. Lorente, M. Persson, Theoretical aspects of tunneling-current-induced bond excitation and breaking at surfaces, Faraday Discuss. (2000) 277-290.

[173] S. Paavilainen, M. Persson, Vibrational assignments and line shapes in inelastic tunneling spectroscopy: H on $\mathrm{Cu}(100)$, Phys. Rev. B 74 (2006). 
[174] A.M. Wodtke, J.C. Tully, D.J. Auerbach, Electronically non-adiabatic interactions of molecules at metal surfaces: can we trust the Born-Oppenheimer approximation for surface chemistry? Int. Rev. Phys. Chem. 23 (2004) 513-539.

[175] M. Silva, R. Jongma, R.W. Field, A.M. Wodtke, The dynamics of "stretched molecules": experimental studies of highly vibrationally excited molecules with stimulated emission pumping, Annu. Rev. Phys. Chem. 52 (2001) 811-852.

[176] A.W. Kleyn, T.C.M. Horn, Rainbow scattering from solid-surfaces, Phys. Rep. 199 (1991) $192-230$.

[177] C.T. Rettner, D.J. Auerbach, J.C. Tully, A.W. Kleyn, Chemical dynamics at the gas-surface interface, J. Phys. Chem. 100 (1996) 13021-13033.

[178] K.C. Janda, J.E. Hurst, C.A. Becker, J.P. Cowin, D.J. Auerbach, L. Wharton, Direct measurement of velocity distributions in argon beam-tungsten surface scattering, J. Chem. Phys. 72 (1980) 24032410 .

[179] J.E. Hurst, L. Wharton, K.C. Janda, D.J. Auerbach, Direct inelastic-scattering Ar from Pt(1 11), J. Chem. Phys. 78 (1983) 1559-1581.

[180] J.E. Hurst, C.A. Becker, J.P. Cowin, K.C. Janda, L. Wharton, D.J. Auerbach, Observation of direct inelastic-scattering in the presence of trapping-desorption scattering - Xe on $\operatorname{Pt}(111)$, Phys. Rev. Lett. 43 (1979) 1175-1177.

[181] J.E. Hurst, L. Wharton, K.C. Janda, D.J. Auerbach, Trapping-desorption scattering of argon from Pt(1 1 1), J. Chem. Phys. 83 (1985) 1376-1381.

[182] M. Korolik, D.W. Arnold, M.J. Johnson, M.M. Suchan, H. Reisler, C. Wittig, Trapping-desorption and direct-inelastic scattering of $\mathrm{HCl}$ from $\mathrm{MgO}(100)$, Chem. Phys. Lett. 284 (1998) 164-170.

[183] G.M. Nathanson, Molecular beam studies of gas-liquid interfaces, Annu. Rev. Phys. Chem. 55 (2004) 231255.

[184] M.E. Saecker, G.M. Nathanson, Collisions of protic and aprotic gases with hydrogen-bonding and hydrocarbon liquids, J. Chem. Phys. 99 (1993) 7056-7075.

[185] B.G. Perkins, D.J. Nesbitt, Quantum-state-resolved $\mathrm{CO}_{2}$ scattering dynamics at the gas-liquid interface: dependence on incident angle, J. Phys. Chem. A 111 (2007) 7420-7430.

[186] B.G. Perkins, D.J. Nesbitt, Quantum-state-resolved $\mathrm{CO}_{2}$ scattering dynamics at the gas-liquid interface: incident collision energy and liquid dependence, J. Phys. Chem. B 110 (2006) 17126-17137.

[187] B.G. Perkins, T. Haber, D.J. Nesbitt, Quantum state-resolved energy transfer dynamics at gas-liquid interfaces: IR laser studies of $\mathrm{CO}_{2}$ scattering from perfluorinated liquids, J. Phys. Chem. B 109 (2005) 16396-16405.

[188] S.A. Vazquez, J.R. Morris, A. Rahaman, O.A. Mazyar, G. Vayner, S.V. Addepalli, W.L. Hase, E. Martinez-Nunez, Inelastic scattering dynamics of Ar from a perfluorinated self-assembled monolayer surface, J. Phys. Chem. A 111 (2007) 12785-12794.

[189] J.R. Lohr, B.S. Day, J.R. Morris, Dynamics of HCl collisions with hydroxyl- and methyl-terminated selfassembled monolayers, J. Phys. Chem. A 110 (2006) 1645-1649.

[190] J.R. Lohr, B.S. Day, J.R. Morris, Scattering, accommodation, and trapping of $\mathrm{HCl}$ in collisions with a hydroxylated self-assembled monolayer, J. Phys. Chem. B 109 (2005) 15469-15475.

[191] B.S. Day, J.R. Morris, Packing density and structure effects on energy-transfer dynamics in argon collisions with organic monolayers, J. Chem. Phys. 122 (2005).

[192] B. Scott Day, J.R. Morris, D. Troya, Classical trajectory study of collisions of Ar with alkanethiolate selfassembled monolayers: potential-energy surface effects on dynamics, J. Chem. Phys. 122 (2005).

[193] B.S. Day, G.M. Davis, J.R. Morris, The effect of hydrogen-bonding and terminal group structure on the dynamics of Ar collisions with self-assembled monolayers, Anal. Chim. Acta 496 (2003) 249-258.

[194] B.S. Day, S.F. Shuler, A. Ducre, J.R. Morris, The dynamics of gas-surface energy exchange in collisions of $\mathrm{Ar}$ atoms with omega-functionalized self-assembled monolayers, J. Chem. Phys. 119 (2003) 8084-8096.

[195] S.F. Shuler, G.M. Davis, J.R. Morris, Energy transfer in rare gas collisions with hydroxyl- and methylterminated self-assembled monolayers, J. Chem. Phys. 116 (2002) 9147-9150.

[196] C.T. Rettner, D.J. Auerbach, J. Lee, Dynamics of the formation of $\mathrm{CD}_{4}$ from the direct reaction of incident D atoms with $\mathrm{CD}_{3} / \mathrm{Cu}(111)$, J. Chem. Phys. 105 (1996) 10115-10122.

[197] C.T. Rettner, D.J. Auerbach, Dynamics of the displacement of CO from Cu(1 1 1) by H atoms incident from the gas phase, J. Chem. Phys. 105 (1996) 8842-8848.

[198] C.T. Rettner, D.J. Auerbach, Dynamics of the formation of HD from D(H) atoms colliding with H(D)/ $\mathrm{Cu}(111)$ : A model study of an Eley-Rideal reaction, Surf. Sci. 358 (1996) 602-608. 
[199] C.T. Rettner, D.J. Auerbach, Dynamics of the Eley-Rideal reaction of D-atoms with H-atoms adsorbed on $\mathrm{Cu}(111)$ - vibrational and rotational state distributions of the HD product, Phys. Rev. Lett. 74 (1995) 4551-4554.

[200] C.T. Rettner, Reaction of an H-atom beam with $\mathrm{Cl} / \mathrm{Au}(111)$ - dynamics of concurrent Eley-Rideal and Langmuir-Hinshelwood mechanisms, J. Chem. Phys. 101 (1994) 1529-1546.

[201] C.T. Rettner, Dynamics of the direct reaction of hydrogen-atoms adsorbed on $\mathrm{Cu}(111)$ with hydrogenatoms incident from the gas-phase, Phys. Rev. Lett. 69 (1992) 383-386.

[202] K.R. Lykke, B.D. Kay, State-to-state inelastic and reactive molecular beam scattering from surfaces, in: N.S. Nogar (Ed.), Laser Photoionization and Desorption Surface Analysis Techniques, SPIE, Bellingham, WA, 1990, pp. 18-29.

[203] E.W. Kuipers, M.G. Tenner, A.W. Kleyn, S. Stolte, Dependence of the NO/Ag(111) trapping probability on molecular-orientation, Chem. Phys. 138 (1989) 451-460.

[204] M.G. Tenner, F.H. Geuzebroek, E.W. Kuipers, A.E. Wiskerke, A.W. Kleyn, S. Stolte, A. Namiki, Orientation dependence of rotational-excitation in NO scattering from $\operatorname{Ag}(111)$, Chem. Phys. Lett. 168 (1990) 45-50.

[205] F.H. Geuzebroek, A.E. Wiskerke, M.G. Tenner, A.W. Kleyn, S. Stolte, A. Namiki, Rotational-excitation of oriented molecules as a probe of molecule surface interaction, J. Phys. Chem. 95 (1991) 8409-8421.

[206] M.G. Tenner, E.W. Kuipers, A.W. Kleyn, S. Stolte, Direct inelastic-scattering of oriented NO from $\operatorname{Ag}(111)$ and $\mathrm{Pt}(111)$, J. Chem. Phys. 94 (1991) 5197-5207.

[207] A.W. Kleyn, Dissociation in molecule surface collisions, J. Phys. - Condens. Matter 4 (1992) 8375-8394.

[208] R. Lahaye, S. Stolte, S. Holloway, A.W. Kleyn, Orientation and energy dependence of NO scattering from Pt(1 11), J. Chem. Phys. 104 (1996) 8301-8311.

[209] M. Brandt, T. Greber, F. Kuhlmann, N. Bowering, U. Heinzmann, State- and orientation-dependent $\mathrm{N}_{2}$ emission in the $\mathrm{N}_{2} \mathrm{O}+$ Cs reaction, Surf. Sci. 404 (1998) 160-164.

[210] G.O. Sitz, A.C. Kummel, R.N. Zare, Alignment and orientation of $\mathrm{N}_{2}$ scattered from $\operatorname{Ag}(111)$, J. Chem. Phys. 87 (1987) 3247-3249.

[211] G.O. Sitz, A.C. Kummel, R.N. Zare, Population and alignment of $\mathrm{N}_{2}$ scattered from $\operatorname{Ag}(111)$, J. Vacuum Sci. Technol. A - Vacuum Surf. Films 5 (1987) 513-517.

[212] A.C. Kummel, G.O. Sitz, R.N. Zare, J.C. Tully, Direct inelastic-scattering of $\mathrm{N}_{2}$ from $\mathrm{Ag}(111)$ ). 3. Normal incident $\mathrm{N}_{2}$, J. Chem. Phys. 89 (1988) 6947-6955.

[213] G.O. Sitz, A.C. Kummel, R.N. Zare, Direct inelastic-scattering of $\mathrm{N}_{2}$ from $\operatorname{Ag}(111)$. 1. Rotational populations and alignment, J. Chem. Phys. 89 (1988) 2558-2571.

[214] G.O. Sitz, A.C. Kummel, R.N. Zare, J.C. Tully, Direct inelastic-scattering of $\mathrm{N}_{2}$ from $\operatorname{Ag}(111) .2$. Orientation, J. Chem. Phys. 89 (1988) 2572-2582.

[215] A.C. Kummel, G.O. Sitz, R.N. Zare, J.C. Tully, Direct inelastic-scattering of $\mathrm{N}_{2}$ from $\operatorname{Ag}(111) .4$. Scattering from high-temperature surface, J. Chem. Phys. 91 (1989) 5793-5801.

[216] K.R. Lykke, B.D. Kay, Rotational rainbows in the inelastic-scattering of $\mathrm{N}_{2}$ from $\mathrm{Au}(111)$, J. Phys. Condens. Matter 3 (1991) S65-S70.

[217] R. Elber, R.B. Gerber, Multiple-collision rotational rainbow effect in molecule-surface scattering, J. Chem. Phys. 79 (1983) 4087-4088.

[218] T. Brunner, W. Brenig, Calculations for the scattering of $\mathrm{N}_{2}$ at a Ag surface, Surf. Sci. 261 (1992) 284-298.

[219] M.A. Hines, R.N. Zare, The interaction of $\mathrm{CO}$ with $\mathrm{Ni}(1111)$ - rainbows and rotational trapping, J. Chem. Phys. 98 (1993) 9134-9147.

[220] A.E. Wiskerke, C.A. Taatjes, A.W. Kleyn, R. Lahaye, S. Stolte, D.K. Bronnikov, B.E. Hayden, Rotational rainbows in NO scattering from $\mathrm{Pt}(111)$, Faraday Discuss. (1993) 297-305.

[221] A.E. Wiskerke, C.A. Taatjes, A.W. Kleyn, R. Lahaye, S. Stolte, D.K. Bronnikov, B.E. Hayden, Survival mechanism for rotational rainbows in highly attractive molecule-surface systems - NO scattering from Pt(1 11), Chem. Phys. Lett. 216 (1993) 93-99.

[222] A.C. Wight, R.E. Miller, Rainbow scattering of methane from $\operatorname{LiF}(100)$ : probing the corrugation and anisotropy of the gas-surface potential, J. Chem. Phys. 109 (1998) 1976-1982.

[223] G.O. Sitz, Gas surface interactions studied with state-prepared molecules, Rep. Prog. Phys. 65 (2002) 11651193.

[224] L.B.F. Juurlink, P.R. McCabe, R.R. Smith, C.L. DiCologero, A.L. Utz, Eigenstate-resolved studies of gassurface reactivity: $\mathrm{CH}_{4}\left(v_{3}\right)$ dissociation on $\mathrm{Ni}(100)$, Phys. Rev. Lett. 83 (1999) 868-871.

[225] L.B.F. Juurlink, R.R. Smith, A.L. Utz, The role of rotational excitation in the activated dissociative chemisorption of vibrationally excited methane on Ni(100), Faraday Discuss. (2000) 147-160. 
[226] L.B.F. Juurlink, R.R. Smith, A.L. Utz, Controlling surface chemistry with light: spatially resolved deposition of rovibrational-state-selected molecules, J. Phys. Chem. B 104 (2000) 3327-3336.

[227] R.D. Beck, P. Maroni, D.C. Papageorgopoulos, T.T. Dang, M.P. Schmid, T.R. Rizzo, Vibrational modespecific reaction of methane on a nickel surface, Science 302 (2003) 98-100.

[228] R.R. Smith, D.R. Killelea, D.F. DelSesto, A.L. Utz, Preference for vibrational over translational energy in a gas-surface reaction, Science 304 (2004) 992-995.

[229] L.B.F. Juurlink, R.R. Smith, D.R. Killelea, A.L. Utz, Comparative study of C-H stretch and bend vibrations in methane activation on Ni(100) and Ni(111), Phys. Rev. Lett. 94 (2005).

[230] P. Maroni, D.C. Papageorgopoulos, M. Sacchi, T.T. Dang, R.D. Beck, T.R. Rizzo, State-resolved gassurface reactivity of methane in the symmetric C-H stretch vibration on Ni(100), Phys. Rev. Lett. 94 (2005).

[231] A.M. Wodtke, H. Yuhui, D.J. Auerbach, Insensitivity of trapping at surfaces to molecular vibration, Chem. Phys. Lett. 413 (2005) 326-330.

[232] M. Korolik, M.M. Suchan, M.J. Johnson, D.W. Arnold, H. Reisler, C. Wittig, Survival of $\mathrm{HCl}(v=2)$ in trapping-desorption from $\mathrm{MgO}(100)$, Chem. Phys. Lett. 326 (2000) 11-21.

[233] M. Gostein, H. Parhikhteh, G.O. Sitz, Survival probability of $\mathrm{H}_{2}(v=1, J=1)$ scattered from $\mathrm{Cu}(110)$, Phys. Rev. Lett. 75 (1995) 342-345.

[234] M. Gostein, G.O. Sitz, Scattering of $\mathrm{H}_{2}(v=1, J=1)$ from $\mathrm{Cu}(110)$ : survival probability versus incident energy, J. Vacuum Sci. Technol. A - Vacuum Surf. Films 14 (1996) 1562-1565.

[235] M. Gostein, G.O. Sitz, Rotational state-resolved sticking coefficients for $\mathrm{H}_{2}$ on $\mathrm{Pd}(111)$ : testing dynamical steering in dissociative adsorption, J. Chem. Phys. 106 (1997) 7378-7390.

[236] M. Gostein, E. Watts, G.O. Sitz, Vibrational relaxation of $\mathrm{H}_{2}(v=1, J=1)$ on $\mathrm{Pd}(111)$, Phys. Rev. Lett. 79 (1997) 2891-2894.

[237] E. Watts, G.O. Sitz, State-to-state scattering in a reactive system: $\mathrm{H}_{2}(v=1, J=1)$ from $\mathrm{Cu}(100)$, J. Chem. Phys. 114 (2001) 4171-4179.

[238] E. Watts, G.O. Sitz, D.A. McCormack, G.J. Kroes, R.A. Olsen, J.A. Groeneveld, J.N.P. Van Stralen, E.J. Baerends, R.C. Mowrey, Rovibrationally inelastic scattering of $(v=1, j=1) \mathrm{H}_{2}$ from $\mathrm{Cu}(100)$ experiment and theory, J. Chem. Phys. 114 (2001) 495-503.

[239] L.C. Shackman, G.O. Sitz, State-to-state scattering of $\mathrm{D}_{2}$ from $\mathrm{Cu}(100)$ and $\mathrm{Pd}(111)$, J. Chem. Phys. 123 (2005).

[240] L.C. Shackman, G.O. Sitz, Rotationally inelastic scattering of HD from $\mathrm{Cu}(100)$ and $\mathrm{Pd}(111)$, J. Chem. Phys. 122 (2005).

[241] G.O. Sitz, C.B. Mullins, Molecular dynamics simulations of the influence of surface temperature on the trapping of methane on iridium single-crystalline surfaces, J. Phys. Chem. B 106 (2002) 8349-8353.

[242] E. Watts, G.O. Sitz, Surface temperature dependence of rotational excitation of $\mathrm{H}_{2}$ scattered from $\mathrm{Pd}(111)$, J. Chem. Phys. 111 (1999) 9791-9796.

[243] A.C. Wight, M. Penno, R.E. Miller, Sequential vibrational relaxation of polyatomic molecules at surfaces: $\mathrm{C}_{2} \mathrm{HD}$ and $\mathrm{C}_{2} \mathrm{H}_{2}$ scattered from $\mathrm{LiF}(001)$, J. Chem. Phys. 111 (1999) 8622-8627.

[244] A.C. Wight, R.E. Miller, Vibrational quenching of acetylene scattered from $\operatorname{LiF}(001)$ : trapping desorption versus direct scattering, J. Chem. Phys. 109 (1998) 8626-8634.

[245] T.W. Francisco, N. Camillone, R.E. Miller, Rotationally inelastic scattering of $\mathrm{C}_{2} \mathrm{H}_{2}$ from $\operatorname{LiF}(100)$ : translational energy dependence, Phys. Rev. Lett. 77 (1996) 1402-1405.

[246] D.C. Jacobs, Reactive collisions of hyperthermal energy molecular ions with solid surfaces, Annu. Rev. Phys. Chem. 53 (2002) 379-407.

[247] J.S. Martin, J.N. Greeley, J.R. Morris, B.T. Feranchak, D.C. Jacobs, Scattering state-selected NO + ON $\mathrm{GaAs}(110)$ - the effect of translational and vibrational-energy on $\mathrm{NO}^{-}$and $\mathrm{O}^{-}$product formation, $\mathrm{J}$. Chem. Phys. 100 (1994) 6791-6812.

[248] J.D. White, J. Chen, D. Matsiev, D.J. Auerbach, A.M. Wodtke, Vibrationally promoted electron emission from low work-function metal surfaces, J. Chem. Phys. 124 (2006).

[249] J.D. White, J. Chen, D. Matsiev, D.J. Auerbach, A.M. Wodtke, Vibrationally promoted emission of electrons from low work function surfaces: oxygen and Cs surface coverage dependence, J. Vacuum Sci. Technol. A 23 (2005) 1085-1089.

[250] J.D. White, J. Chen, D. Matsiev, D.J. Auerbach, A.M. Wodtke, Conversion of large-amplitude vibration to electron excitation at a metal surface, Nature 433 (2005) 503-505.

[251] A.M. Wodtke, Y.H. Huang, D.J. Auerbach, Interaction of NO $(v=12)$ with $\operatorname{LiF}(001)$ : evidence for anomalously large vibrational relaxation rates, J. Chem. Phys. 118 (2003) 8033-8041. 
[252] Y.H. Huang, C.T. Rettner, D.J. Auerbach, A.M. Wodtke, Vibrational promotion of electron transfer, Science 290 (2000) 111-114.

[253] H. Hou, C.T. Rettner, D.J. Auerbach, Y. Huang, S.J. Gulding, A.M. Wodtke, The interaction of highly vibrationally excited molecules with surfaces: vibrational relaxation and reaction of $\mathrm{NO}(v)$ at $\mathrm{Cu}(1111)$ and O/Cu(1 11 ), Faraday Discuss. (1999) 181-200.

[254] H. Hou, Y. Huang, S.J. Gulding, C.T. Rettner, D.J. Auerbach, A.M. Wodtke, Enhanced reactivity of highly vibrationally excited molecules on metal surfaces, Science 284 (1999) 1647-1650.

[255] H. Hou, Y. Huang, S.J. Gulding, C.T. Rettner, D.J. Auerbach, A.M. Wodtke, Direct multiquantum relaxation of highly vibrationally excited NO in collisions with $\mathrm{O} / \mathrm{Cu}(111)$, J. Chem. Phys. 110 (1999) $10660-10663$.

[256] J. Chen, D. Matsiev, J.D. White, M. Murphy, A.M. Wodtke, Hexapole transport and focusing of vibrationally excited NO molecules prepared by optical pumping, Chem. Phys. 301 (2004) 161-172.

[257] X.M. Yang, A.M. Wodtke, Controlling the quantum numbers in chemical-reactions - reactivity and energy-transfer of highly vibrationally excited molecules, Int. Rev. Phys. Chem. 12 (1993) 123-147.

[258] X.M. Yang, A.M. Wodtke, State-to-state spin orbit and rotational energy-transfer of very highly vibrationally excited nitric-oxide, J. Chem. Phys. 96 (1992) 5123-5128.

[259] X.M. Yang, E.H. Kim, A.M. Wodtke, The vibrational quantum number dependence of the collisional lifetime in nitric-oxide self-relaxation up to $v^{\prime}=25$, J. Chem. Phys. 93 (1990) 4483-4484.

[260] X. Yang, A.M. Wodtke, Efficient state-specific preparation of highly vibrationally excited $\operatorname{NO}\left(X^{2} \Phi\right), J$. Chem. Phys. 92 (1990) 116-120.

[261] J.A. Mack, K. Mikulecky, A.M. Wodtke, Resonant vibration-vibration energy transfer between highly vibrationally excited $\mathrm{O}_{2}\left(X^{3} \Sigma_{g}^{-}, v=15-26\right)$ and $\mathrm{CO}_{2}, \mathrm{~N}_{2} \mathrm{O}, \mathrm{N}_{2}$, and $\mathrm{O}_{3}$, J. Chem. Phys. 105 (1996) 4105-4116.

[262] R. Toumi, P.L. Houston, A.M. Wodtke, Reactive $\mathrm{O}_{2}(v>=26)$ as a source of stratospheric $\mathrm{O}_{3}$, J. Chem. Phys. 104 (1996) 775-776.

[263] B.D. Kay, T.D. Raymond, M.E. Coltrin, Observation of a dynamic propensity rule in rotationally inelastic gas-surface scattering - $\mathrm{NH}_{3}$ on $\mathrm{Au}(111)$, Phys. Rev. B 36 (1987) 6695-6697.

[264] B.D. Kay, T.D. Raymond, M.E. Coltrin, Observation of direct multiquantum vibrational-excitation in gassurface scattering $-\mathrm{NH}_{3}$ on $\mathrm{Au}(111)$, Phys. Rev. Lett. 59 (1987) 2792-2794.

[265] C.T. Rettner, F. Fabre, J. Kimman, D.J. Auerbach, Observation of direct vibrational-excitation in gassurface collisions - NO on Ag(111), Phys. Rev. Lett. 55 (1985) 1904-1907.

[266] A.M. Wodtke, Y.H. Huang, D.J. Auerbach, Non-Arrhenius surface temperature dependence in vibrational excitation of $\mathrm{NO}$ on $\mathrm{Au}(111)$ : possible evidence for the importance of surface electronic states, Chem. Phys. Lett. 364 (2002) 231-236.

[267] E.K. Watts, J.L.W. Siders, G.O. Sitz, Vibrational excitation of NO scattered from $\mathrm{Cu}(110)$, Surf. Sci. 374 (1997) 191-196.

[268] G.A. Gates, S. Holloway, The vibrational-excitation of NO/Ag, Surf. Sci. 309 (1994) 132-137.

[269] R. Brako, The mechanism of vibrational-excitation in NO scattering on $\operatorname{Ag}(111)$, Surf. Sci. 249 (1991) L312-L316.

[270] Z.S. Wang, G.R. Darling, S. Holloway, The surface temperature dependence of the inelastic scattering and dissociation of hydrogen molecules from metal surfaces, J. Chem. Phys. 120 (2004) 2923-2933.

[271] G.A. Gates, G.R. Darling, S. Holloway, A theoretical-study of the vibrational-excitation of NO/Ag(111), J. Chem. Phys. 101 (1994) 6281-6288.

[272] A. Gross, W. Brenig, Vibrational-excitation of NO in NOAg scattering revisited, Surf. Sci. 289 (1993) 335339.

[273] Y. Huang, A.M. Wodtke, H. Hou, C.T. Rettner, D.J. Auerbach, Observation of vibrational excitation and deexcitation for $\mathrm{NO}(v=2)$ scattering from $\mathrm{Au}(111)$ : Evidence for electron-hole-pair mediated energy transfer, Phys. Rev. Lett. 84 (2000) 2985-2988.

[274] M. Cizek, J. Horacek, W. Domcke, Nuclear dynamics of the $\mathrm{H}_{2}^{-}$collision complex beyond the local approximation: associative detachment and dissociative attachment to rotationally and vibrationally excited molecules, J. Phys. B - Atom. Mol. Opt. Phys. 31 (1998) 2571-2583.

[275] A. Hellman, B. Razaznejad, B.I. Lundqvist, Trends in sticking and adsorption of diatomic molecules on the Al(1 11 1) surface, Phys. Rev. B 71 (2005).

[276] A. Hellman, B. Razaznejad, Y. Yourdshahyan, H. Ternow, I. Zoric, B.I. Lundqvist, Initial sticking of $\mathrm{O}_{2}$ modeled by nonadiabatic charge transfer, Surf. Sci. 532 (2003) 126-131.

[277] G.J. Kroes, M.F. Somers, Six-dimensional dynamics of dissociative chemisorption of $\mathrm{H}_{2}$ on metal surfaces, J. Theor. Computat. Chem. 4 (2005) 493-581. 
[278] G.J. Kroes, A. Gross, E.J. Baerends, M. Scheffler, D.A. McCormack, Quantum theory of dissociative chemisorption on metal surfaces, Acc. Chem. Res. 35 (2002) 193-200.

[279] G.J. Kroes, Six-dimensional quantum dynamics of dissociative chemisorption of $\mathrm{H}_{2}$ on metal surfaces, Prog. Surf. Sci. 60 (1999) 1-85.

[280] P. Nieto, E. Pijper, D. Barredo, G. Laurent, R.A. Olsen, E.J. Baerends, G.J. Kroes, D. Farias, Reactive and nonreactive scattering of $\mathrm{H}_{2}$ from a metal surface is electronically adiabatic, Science 312 (2006) 86-89.

[281] C. Diaz, J.K. Vincent, G.P. Krishnamohan, R.A. Olsen, G.J. Kroes, K. Honkala, J.K. Norskov, Reactive and nonreactive scattering of $\mathrm{N}_{2}$ from $\mathrm{Ru}(0001)$ : a six-dimensional adiabatic study, J. Chem. Phys. 125 (2006).

[282] K.R. Lykke, B.D. Kay, Rotationally inelastic gas-surface scattering - $\mathrm{HCl}$ from $\mathrm{Au}(111)$, J. Chem. Phys. 92 (1990) 2614-2623.

[283] Q. Ran, D. Matsiev, D.J. Auerbach, A.M. Wodtke, An advanced molecule-surface scattering instrument for study of vibrational energy transfer in gas-solid collisions, Rev. Sci. Instrum. 78 (2007) 104104.

[284] Q. Ran, D. Matsiev, D.J. Auerbach, A.M. Wodtke, Direct translation-to-vibrational energy transfer of HCl on gold: measurement of absolute vibrational excitation probabilities, Nucl. Instr. Methods Phys. Res. Section B - Beam Interact. Mater. Atoms 258 (2007) 1-6.

[285] Q. Ran, D. Matsiev, D.J. Auerbach, A.M. Wodtke, Observation of a change of vibrational excitation mechanism with surface temperature: $\mathrm{HCl}$ collisions with $\mathrm{Au}(111)$, Phys. Rev. Lett. 98 (2007).

[286] D.V. Shalashilin, B. Jackson, M. Persson, Eley-Rideal and hot-atom dynamics of HD formation by H(D) incident from the gas phase on $\mathrm{D}(\mathrm{H})$-covered $\mathrm{Cu}(111)$, Faraday Discuss. (1998) 287-300.

[287] B. Jackson, M. Persson, B.D. Kay, Quantum-mechanical study of $\mathrm{H}(\mathrm{g})+\mathrm{Cl}-\mathrm{Au}(111)$ - Eley-Rideal mechanism, J. Chem. Phys. 100 (1994) 7687-7695.

[288] D. Lemoine, J.G. Quattrucci, B. Jackson, Efficient Eley-Rideal reactions of $\mathrm{H}$ atoms with single $\mathrm{Cl}$ adsorbates on $\mathrm{Au}(111)$, Phys. Rev. Lett. 89 (2002).

[289] J.G. Quattrucci, B. Jackson, D. Lemoine, Eley-Rideal reactions of $\mathrm{H}$ atoms with $\mathrm{Cl}$ adsorbed on $\mathrm{Au}\left(\begin{array}{lll}1 & 1 & 1\end{array}\right)$ : quantum and quasiclassical studies, J. Chem. Phys. 118 (2003) 2357-2366.

[290] J.G. Quattrucci, B. Jackson, Quasiclassical study of Eley-Rideal and hot atom reactions of $\mathrm{H}$ atoms with $\mathrm{Cl}$ adsorbed on a $\mathrm{Au}(111)$ surface, J. Chem. Phys. 122 (2005).

[291] L. Diekhoner, H. Mortensen, A. Baurichter, A.C. Luntz, B. Hammer, Dynamics of high-barrier surface reactions: laser-assisted associative desorption of $\mathrm{N}_{2}$ from $\mathrm{Ru}(0001)$, Phys. Rev. Lett. 84 (2000) 4906-4909.

[292] KROES, private communication.

[293] H. Nienhaus, Electronic excitations by chemical reactions on metal surfaces, Surf. Sci. Rep. 45 (2002) $3-78$.

[294] T. Greber, Nonadiabatic gas surface reactions, Curr. Opin. Solid State Mater. Sci. 3 (1998) 446-450.

[295] T. Greber, Charge-transfer induced particle emission in gas surface reactions, Surf. Sci. Rep. 28 (1997) 1-64.

[296] T. Greber, Chemical hole diving, Chem. Phys. Lett. 222 (1994) 292-296.

[297] B. Kasemo, Photon emission during chemisorption of oxygen on Al and Mg surfaces, Phys. Rev. Lett. 32 (1974) 1114-1117.

[298] L.E. Brus, J. Comas, Chemisorptive luminescence - oxygen on Si(111) surfaces, J. Chem. Phys. 54 (1971) 2771-2776.

[299] B. McCarrol, Chemisorptive luminescence, J. Chem. Phys. 50 (1969) 4758.

[300] H.U. Suter, T. Greber, On the dissociation of $\mathrm{N}_{2} \mathrm{O}$ after electron attachment, J. Phys. Chem. B 108 (2004) 14511-14517.

[301] M. Brandt, T. Greber, N. Bowering, U. Heinzmann, The role of molecular state and orientation in harpooning reactions: $\mathrm{N}_{2} \mathrm{O}$ on $\mathrm{Cs} / \mathrm{Pt}(111)$, Phys. Rev. Lett. 81 (1998) 2376-2379.

[302] A. Bottcher, R. Grobecker, T. Greber, G. Ertl, Negative particle-emission from a $\mathrm{Cs} / \mathrm{Ru}(0001)$ surface during exposure to $\mathrm{NO}$ and $\mathrm{NO}_{2}$, Chem. Phys. Lett. 208 (1993) 404- 408.

[303] R. Grobecker, H. Shi, H. Bludau, T. Hertel, T. Greber, A. Bottcher, K. Jacobi, G. Ertl, Emission of exoelectrons during oxidation of Cs via thermal-activation of a metastable $\mathrm{O}_{2}$ - surface species, Phys. Rev. Lett. 72 (1994) 578-581.

[304] R. Grobecker, T. Greber, A. Bottcher, G. Ertl, Thermally activated emission of exoelectrons accompanying the oxidation of Cs films, Phys. Status Solidi A - Appl. Res. 146 (1994) 259-267.

[305] A. Bottcher, R. Grobecker, R. Imbeck, A. Morgante, G. Ertl, Exoelectron emission during oxidation of Cs films, J. Chem. Phys. 95 (1991) 3756-3766.

[306] A. Bottcher, R. Imbeck, A. Morgante, G. Ertl, Nonadiabatic surface-reaction - mechanism of electronemission in the Cs $+\mathrm{O}_{2}$ system, Phys. Rev. Lett. 65 (1990) 2035-2037. 
[307] T. Greber, K. Freihube, R. Grobecker, A. Bottcher, K. Hermann, G. Ertl, D. Fick, Nonadiabatic processes during the oxidation of Li layers, Phys. Rev. B 50 (1994) 8755-8762.

[308] K. Hermann, K. Freihube, T. Greber, A. Bottcher, R. Grobecker, D. Fick, G. Ertl, Dynamics of the interaction of $\mathrm{O}_{2}$ with Li surfaces, Surf. Sci. 313 (1994) L806-L810.

[309] T. Greber, R. Grobecker, A. Morgante, A. Bottcher, G. Ertl, O-escape during the oxidation of cesium, Phys. Rev. Lett. 70 (1993) 1331-1334.

[310] A. Bottcher, A. Morgante, G. Ertl, Ejection of O-ions by interaction of $\mathrm{O}_{2}$ with $\mathrm{Ru}(0001)$ covered with submonolayers of Cs, Surf. Sci. 359 (1996) L461-L466.

[311] A. Bottcher, A. Morgante, T. Giessel, T. Greber, G. Ertl, Exoelectron emission at Cs surfaces by accelerated $\mathrm{O}_{2}$ molecules, Chem. Phys. Lett. 231 (1994) 119-122.

[312] J.K. Nørskov, B.I. Lundqvist, Secondary-ion emission probability in sputtering, Phys. Rev. B 19 (1979) 5661-5665.

[313] H.S. Bergh, B. Gergen, H. Nienhaus, A. Majumdar, W.H. Weinberg, E.W. McFarland, An ultrahigh vacuum system for the fabrication and characterization of ultrathin metal-semiconductor films and sensors, Rev. Sci. Instrum. 70 (1999) 2087-2094.

[314] H. Nienhaus, H.S. Bergh, B. Gergen, A. Majumdar, W.H. Weinberg, E.V. McFarland, Ultrathin Cu films on Si(1 1 1): Schottky barrier formation and sensor applications, J. Vacuum Sci. Technol. A - Vacuum Surf. Films 17 (1999) 1683-1687.

[315] H. Nienhaus, H.S. Bergh, B. Gergen, A. Majumdar, W.H. Weinberg, E.W. McFarland, Selective H atom sensors using ultrathin Ag Si Schottky diodes, Appl. Phys. Lett. 74 (1999) 4046-4048.

[316] H. Nienhaus, H.S. Bergh, B. Gergen, A. Majumdar, W.H. Weinberg, E.W. McFarland, Electron-hole pair creation at $\mathrm{Ag}$ and $\mathrm{Cu}$ surfaces by adsorption of atomic hydrogen and deuterium, Phys. Rev. Lett. 82 (1999) 446-449.

[317] H. Nienhaus, H.S. Bergh, B. Gergen, A. Majumdar, W.H. Weinberg, E.W. McFarland, Direct detection of electron-hole pairs generated by chemical reactions on metal surfaces, Surf. Sci. 445 (2000) 335-342.

[318] B. Gergen, H. Nienhaus, W.H. Weinberg, E.W. McFarland, Chemically induced electronic excitations at metal surfaces, Science 294 (2001) 2521-2523.

[319] B. Gergen, S.J. Weyers, H. Nienhaus, W.H. Weinberg, E.W. McFarland, Observation of excited electrons from nonadiabatic molecular reactions of $\mathrm{NO}$ and $\mathrm{O}_{2}$ on polycrystalline Ag, Surf. Sci. 488 (2001) 123-132.

[320] H. Nienhaus, B. Gergen, W.H. Weinberg, E.W. McFarland, Detection of chemically induced hot charge carriers with ultrathin metal film Schottky contacts, Surf. Sci. 514 (2002) 172-181.

[321] H. Nienhaus, S.J. Weyers, B. Gergen, E.W. McFarland, Thin Au/Ge Schottky diodes for detection of chemical reaction induced electron excitation, Sensor. Actuator. B - Chem. 87 (2002) 421-424.

[322] B.R. Cuenya, H. Nienhaus, E.W. McFarland, Chemically induced charge carrier production and transport in $\mathrm{Pd} / \mathrm{SiO}_{2} / \mathrm{n}-\mathrm{Si}(111)$ metal-oxide-semiconductor Schottky diodes, Phys. Rev. B 70 (2004).

[323] S. Glass, H. Nienhaus, Continuous monitoring of Mg oxidation by internal exoemission, Phys. Rev. Lett. 93 (2004).

[324] D. Krix, R. Nuenthel, H. Nienhaus, Chemical interaction of H and D atoms with Ag/H:p-Si(1 1 1) thin film diodes, J. Vacuum Sci. Technol. A 25 (2007) 1156-1160.

[325] D. Krix, R. Nunthel, H. Nienhaus, Generation of hot charge carriers by adsorption of hydrogen and deuterium atoms on a silver surface, Phys. Rev. B 75 (2007) 073410.

[326] H. Nienhaus, D. Krix, S. Glass, Varying the Schottky barrier of thin film Mg/H:p-Si(1 11 ) contacts: properties and applications, J. Vacuum Sci. Technol. A 25 (2007) 950-954.

[327] J.Y. Park, J.R. Renzas, A.M. Contreras, G.A. Somorjai, The genesis and importance of oxide-metal interface controlled heterogeneous catalysis; the catalytic nanodiode, Top. Catal. 46 (2007) 217-222.

[328] G.A. Somorjai, K.M. Bratlie, M.O. Montano, J.Y. Park, Dynamics of surface catalyzed reactions; the roles of surface defects, surface diffusion, and hot electrons, J. Phys. Chem. B 110 (2006) 20014-20022.

[329] J.Y. Park, G.A. Somorjai, The catalytic nanodiode: detecting continous electron flow at oxide-metal interfaces generated by a gas-phase exothermic reaction, Chemphyschem 7 (2006) 1409-1413.

[330] J.Y. Park, G.A. Somorjai, Energy conversion from catalytic reaction to hot electron current with metalsemiconductor Schottky nanodiodes, J. Vacuum Sci. Technol. B 24 (2006) 1967-1971.

[331] G.A. Somorjai, The catalytic nanodiode. Its role in catalytic reaction mechanisms in a historical perspective, Catal. Lett. 101 (2005) 1-3.

[332] X.Z. Ji, A. Zuppero, J.M. Gidwani, G.A. Somorjai, Electron flow generated by gas phase exothermic catalytic reactions using a platinum-gallium nitride nanodiode, J. Am. Chem. Soc. 127 (2005) 57925793 . 
[333] X.Z. Ji, A. Zuppero, J.M. Gidwani, G.A. Somorjai, The catalytic nanodiode: gas phase catalytic reaction generated electron flow using nanoscale platinum titanium oxide Schottky diodes, Nano Lett. 5 (2005) $753-$ 756.

[334] X.Z. Ji, G.A. Somorjai, Continuous hot electron generation in $\mathrm{Pt} / \mathrm{TiO}_{2}, \mathrm{Pd} / \mathrm{TiO}_{2}$, and $\mathrm{Pt} / \mathrm{GaN}$ catalytic nanodiodes from oxidation of carbon monoxide, J. Phys. Chem. B 109 (2005) 2253022535 .

[335] E.W. McFarland, J. Tang, A photovoltaic device structure based on internal electron emission, Nature 421 (2003) 616-618.

[336] J. Tang, H. Birkedal, E.W. McFarland, G.D. Stucky, Self-assembly of CdSe/CdS quantum dots by hydrogen bonding on Au surfaces for photoreception, Chem. Commun. (2003) 2278-2279.

[337] J. Tang, M. White, G.D. Stucky, E.W. McFarland, Electrochemical fabrication of large-area $\mathrm{Au} / \mathrm{TiO}_{2}$ junctions, Electrochem. Commun. 5 (2003) 497-501.

[338] J.W. Gadzuk, On the detection of chemically-induced hot electrons in surface processes: from X-ray edges to Schottky barriers, J. Phys. Chem. B 106 (2002) 8265-8270.

[339] D.M. Bird, M. Persson, J.R. Trail, S. Holloway, Dynamics of the spin transition in the adsorption of hydrogen atoms on metals, Surf. Sci. 566 (2004) 761-766.

[340] M. Lindenblatt, E. Pehlke, Time-dependent density-functional molecular-dynamics study of the isotope effect in chemicurrents, Surf. Sci. 600 (2006) 5068-5073.

[341] M. Lindenblatt, E. Pehlke, Ab initio simulation of the spin transition during chemisorption: H/Al(1 111$)$, Phys. Rev. Lett. 97 (2006).

[342] M. Lindenblatt, J. van Heys, E. Pehlke, Molecular dynamics of nonadiabatic processes at surfaces: chemisorption of H/Al(111), Surf. Sci. 600 (2006) 3624-3628.

[343] M. Lindenblatt, E. Pehlke, A. Duvenbeck, B. Rethfeld, A. Wucher, Kinetic excitation of solids: the concept of electronic friction, Nucl. Instr. Methods Phys. Res. Section B - Beam Interact. Mater. Atoms 246 (2006) 333-339.

[344] J. Van Heys, M. Lindenblatt, E. Pehlke, Molecular-dynamics simulations of non-adiabatic processes at surfaces, Phase Trans. 78 (2005) 773-786.

[345] C. Corriol, G.R. Darling, S. Holloway, Computational studies of nonadiabatic effects in gas-surface encounters, Isreal J. Chem. 45 (2005) 1-12.

[346] L. Osterlund, I. Zoric, B. Kasemo, Dissociative sticking of $\mathrm{O}_{2}$ on Al(111), Phys. Rev. B 55 (1997) 1545215455.

[347] J. Behler, B. Delley, S. Lorenz, K. Reuter, M. Scheffler, Dissociation of $\mathrm{O}_{2}$ at $\mathrm{Al}(111)$ : the role of spin selection rules, Phys. Rev. Lett. 94 (2005).

[348] H. Brune, J. Wintterlin, R.J. Behm, G. Ertl, Surface migration of hot adatoms in the course of dissociative chemisorption of oxygen on $\mathrm{Al}(111)$, Phys. Rev. Lett. 68 (1992) 624-626.

[349] A.J. Komrowski, J.Z. Sexton, A.C. Kummel, M. Binetti, O. Weisse, E. Hasselbrink, Oxygen abstraction from dioxygen on the $\mathrm{Al}(111)$ surface, Phys. Rev. Lett. 8724 (2001).

[350] M. Binetti, O. Weisse, E. Hasselbrink, A.J. Komrowski, A.C. Kummel, Abstractive chemisorption of $\mathrm{O}_{2}$ on $\mathrm{Al}(111)$, Faraday Discuss. (2000) 313-320.

[351] J. Kerwin, X.W. Sha, B. Jackson, Classical studies of H atom trapping on a graphite surface, J. Phys. Chem. B 110 (2006) 18811-18817.

[352] X.W. Sha, B. Jackson, D. Lemoine, B. Lepetit, Quantum studies of H atom trapping on a graphite surface, J. Chem. Phys. 122 (2005).

[353] T. Zecho, A. Guttler, X.W. Sha, B. Jackson, J. Kuppers, Adsorption of hydrogen and deuterium atoms on the (0001) graphite surface, J. Chem. Phys. 117 (2002) 8486-8492.

[354] T. Zecho, A. Guttler, X.W. Sha, D. Lemoine, B. Jackson, J. Kuppers, Abstraction of D chemisorbed on graphite(0001) with gaseous H atoms, Chem. Phys. Lett. 366 (2002) 188-195.

[355] X.W. Sha, B. Jackson, D. Lemoine, Quantum studies of Eley-Rideal reactions between H atoms on a graphite surface, J. Chem. Phys. 116 (2002) 7158-7169.

[356] M. Durr, U. Hofer, Dissociative adsorption of molecular hydrogen on silicon surfaces, Surf. Sci. Rep. 61 (2006) 465-526.

[357] S.C. Park, W.K. Park, J.M. Bowman, Classical and quantum mechanical studies of the CO vibrations in $\mathrm{CO} / \mathrm{Cu}(100)$, Surf. Sci. 428 (1999) 343-348.

[358] S. Carter, S.J. Culik, J.M. Bowman, Vibrational self-consistent field method for many-mode systems: a new approach and application to the vibrations of $\mathrm{CO}$ adsorbed on $\mathrm{Cu}(100)$, J. Chem. Phys. 107 (1997) 1045810469. 
[359] J.K. Norskov, B.I. Lundqvist, Correlation between sticking probability and adsorbate-induced electronstructure, Surf. Sci. 89 (1979) 251-261.

[360] B.R. Trenhaile, V.N. Antonov, G.J. Xu, A. Agrawal, A.W. Signor, R.E. Butera, K.S. Nakayama, J.H. Weaver, Phonon-activated electron-stimulated desorption of halogens from $\operatorname{Si}(100)-(2 \times 1)$, Phys. Rev. B 73 (2006).

[361] B.R. Trenhaile, V.N. Antonov, G.J. Xu, K.S. Nakayama, J.H. Weaver, Electron-stimulated desorption from an unexpected source: internal hot electrons for $\mathrm{Br}-\mathrm{Si}(100)-(2 \times 1)$, Surf. Sci. 583 (2005) L135-L141.

[362] R. van Harrevelt, K. Honkala, J.K. Norskov, U. Manthe, The reaction rate for dissociative adsorption of $\mathrm{N}_{2}$ on stepped $\mathrm{Ru}(0001)$ : six-dimensional quantum calculations, J. Chem. Phys. 122 (2005).

[363] S. Dahl, E. Tornqvist, I. Chorkendorff, Dissociative adsorption of $\mathrm{N}_{2}$ on $\mathrm{Ru}(0001)$ : a surface reaction totally dominated by steps, J. Catal. 192 (2000) 381-390.

[364] S. Dahl, A. Logadottir, R.C. Egeberg, J.H. Larsen, I. Chorkendorff, E. Tornqvist, J.K. Norskov, Role of steps in $\mathrm{N}_{2}$ activation on $\mathrm{Ru}(0001)$, Phys. Rev. Lett. 83 (1999) 1814-1817.

[365] B. Hammer, Bond activation at monatomic steps: NO dissociation at corrugated Ru(0001), Phys. Rev. Lett. 83 (1999) 3681-3684.

[366] T. Zambelli, J. Wintterlin, J. Trost, G. Ertl, Identification of the "active sites" of a surface-catalyzed reaction, Science 273 (1996) 1688-1690.

[367] P. Gambardella, Z. Sljivancanin, B. Hammer, M. Blanc, K. Kuhnke, K. Kern, Oxygen dissociation at Pt steps, Phys. Rev. Lett. 8705 (2001).

[368] M. Haruta, Size- and support-dependency in the catalysis of gold, Catal. Today 36 (1997) 153-166.

[369] M. Haruta, S. Tsubota, T. Kobayashi, H. Kageyama, M.J. Genet, B. Delmon, Low-temperature oxidation of $\mathrm{CO}$ over gold supported on $\mathrm{TiO}_{2}$, alpha- $\mathrm{Fe}_{2} \mathrm{O}_{3}$, and $\mathrm{CO}_{3} \mathrm{O}_{4}$, J. Catal. 144 (1993) 175-192.

[370] M. Haruta, N. Yamada, T. Kobayashi, S. Iijima, Gold catalysts prepared by coprecipitation for lowtemperature oxidation of hydrogen and of carbon-monoxide, J. Catal. 115 (1989) 301-309.

[371] M. Haruta, T. Kobayashi, H. Sano, N. Yamada, Novel gold catalysts for the oxidation of carbonmonoxide at a temperature far below 0-degrees-C, Chem. Lett. (1987) 405-408. 